\title{
Structural and Functional Investigations of the Protein Synthesis in Saccharomyces cerevisiae
}

PhD Thesis

\author{
in partial fulfilment of the requirements \\ for the degree "Doctor of Philosophy" (PhD) \\ in the Molecular Biology Program \\ at the Georg-August-University Gttingen, \\ Faculty of Biology
}

submitted by

Sohail Khoshnevis

born in

Hamedan, Iran

Göttingen, 2010 
To my father... 


\section{Thesis committee:}

Prof. Dr. Ralf Ficner

Georg-August-Universität Göttingen

Institute for Microbiology and Genetic

Department of Molecular Structural Biology

Prof. Dr. Holger Stark

Max Planck Institute for Biophysical Chemistry

3D Electron Microscopy Group

Prof. Dr. Marina Rodnina

Max Planck Institute for Biophysical Chemistry

Department of Physical Biochemistry 


\section{Affidavit}

I hereby declare that this PhD thesis 'Structural and Functional Investigations of the Protein Synthesis in Saccharomyces cerevisiae' has been written independently with no other aids or sources than quoted. This thesis (wholly or in part) has not been submitted elsewhere for any academic award or qualification.

Sohail Khoshnevis

October 2010

Göttingen, Germany 


\section{Preface}

The following thesis summarizes my work on eukaryotic initiation factor 3 (elF3) in yeast, which was performed at the University of Göttingen between October 2006 and October 2010. The work was performed under the supervision of Prof. Dr. Ralf Ficner. The work led to the following publications:

Khoshnevis, S., Gross, T., Rotte, C., Baierlein, C., Ficner, R., and Krebber, H. 2010. The ironsulphur protein RNase $L$ inhibitor functions in translation termination. EMBO reports 11: 214-9.

Khoshnevis, S., Neumann, P., and Ficner, R. 2010. Crystal Structure of the RNA Recognition Motif of Yeast Translation Initiation Factor elF3b Reveals Differences to Human elF3b. PLoS ONE 5(9): e12784.

Khoshnevis, S., Milon, P., Hauer, F., Schmidt, B., Stark, H., Rodnina, M., and Ficner, R. Reconstitution and preliminary structural and kinetic insights into yeast elF3. Manuscript in preparation.

Results of the work described herein are presented in the international meetings as listed below.

Khoshnevis, S., and Ficner, R. Structural insights into yeast elF3. EMBO Conference on Protein Synthesis and Translational Control. Heidelberg, Germany, September 2009.

Khoshnevis, S., Gross, T., Rotte, C., Baierlein, C., Ficner, R., and Krebber, H. The iron-sulphur protein RNase $\mathrm{L}$ inhibitor functions in translation termination. Ribosome Meeting. Orvieto, Italy, May 2010.

Khoshnevis, S., Neumann, P., and Ficner, R. Crystal Structure of the RNA Recognition Motif of Yeast Translation Initiation Factor elF3b Reveals Differences to Human. Murnau Conference on Structural Biology. Murnau, Germany, October 2010. 


\section{Table of Contents}

1

1.1

1.2

1.2.1

1.2 .2

1.2 .2 .1

1.2.2.2

1.2.2.4

1.2.2.5

1.2.2.5.4

1.2.2.5.5

1.2 .2 .5 .6

1.3

1.4

1.5

2

2.1

2.2
Introduction

A brief overview of protein synthesis

Eukaryotic translation initiation. 3

Flow of translation initiation............................................ 3

The role of initiation factors........................................... 4

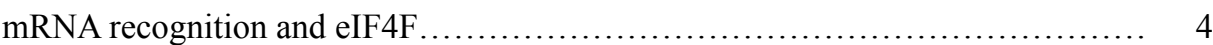

Role of eIF1, eIF1A and eIF5B in scanning, start codon selection and subunit joining....

eIF2 and initiator tRNA delivery to the $40 \mathrm{~S}$

10

eIF5, the GTPase activating protein

12

eIF3; a versatile scaffold.

Role of eIF3 in mRNA recruitment and scanning.

15

Ribosome binding by eIF3....

The sub-stoichiometric $\mathrm{j}$ subunit

The role of eIF3 in ribosome dissociation.

Modularity of eIF3

eIF3, proteasome and COP9 signalosome.

Translational control at initiation level.

eIF2 phosphorylation and translation of uORFs

20

Translational control by affecting cap recognition.......................... 21

Translational control via mRNA binding proteins $\ldots \ldots \ldots \ldots \ldots \ldots \ldots \ldots \ldots \ldots \ldots, 21$

Translational control via microRNAs....................................... 22

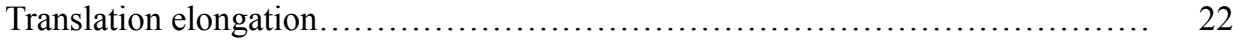

Translation termination and ribosomal recycling ............................ 23

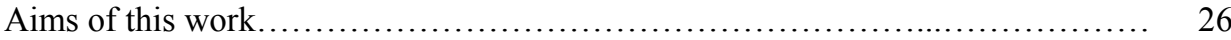

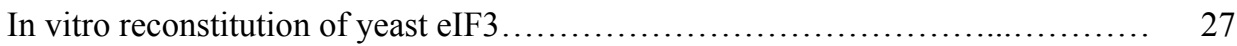

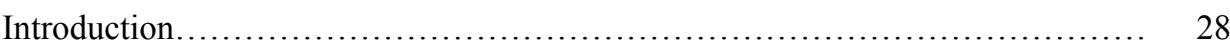

Results.............................................................. 30 
Discussion

Preliminary kinetic insights into the formation of eIF3 and its binding to the 40S subunit using in vitro reconstituted fluorescence-labeled eIF3.

Bibliography.

Acknowledgement.

Curriculum Vitae. 


\section{Abstract}

Protein synthesis is an important house-keeping activity of all the cells. It follows the same concepts in all three domains of life: the genetic code is deciphered and transcribed into the mRNA, which in turn is translated into the polypeptide by the help of the ribosome. Translation of proceeds through four steps: initiation, elongation, termination and ribosome recycling. Despite following the same concepts, different steps of translation show certain domainspecificities. One of the hall-marks of eukaryotic initiation, is the complex initiation step, requiring intricate interaction of several protein factors called eukaryotic initiation factors (eIFs).

The largest eukaryotic initiation factor, eIF3, is a multi-subunit complex which serves as a scaffold to which other initiation factors bind. It facilitates mRNA recruitment and assembly of other initiation factors on the $40 \mathrm{~S}$ ribosomal subunit. Its complex and flexible nature hinders its purification for structural and biochemical studies. The major part of the present work, which is presented in chapter two, was to establish a protocol for recombinant purification and in vitro assembly of Saccharomyces cerevisiae eIF3. This complex was subjected to structural studies by single-particle electron microscopy. Initial results were obtained regarding positioning of eIF3 on the $40 \mathrm{~S}$ subunit, showing its binding to the solvent exposed side of the small ribosome subunit. In vitro reconstitution of eIF3, in combination with Limited proteolysis and massspectrometry, allowed the formation and analysis of its different subcomplexes and stable fragments which form the core of eIF3 complex. In addition, fluorescence-labeling of eIF3 introduced new strategy for studying the kinetics of translation initiation and order of complex formation.

One of the large subunits of eIF3, eIF3b/Prt1, serves as a scaffold within eIF3 as it interacts with

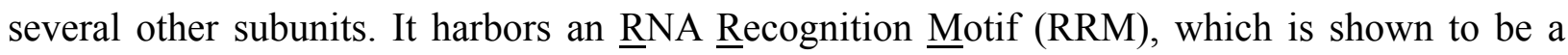
non-canonical RRM in human as it is not capable to interact with oligonucleotides, but rather interacts with eIF3j/Hcr1, a sub-stoichiometric subunit of eIF3. In chapter three, the highresolution crystal structure of the eIF3b RRM domain from yeast is presented. It exhibits the same fold as its human ortholog. Thermodynamic analysis of the interaction between yeast eIF3b-RRM and eIF3j as well the conservation of the eIF3j binding site between human and yeast eIF3b-RRM suggested that the same mode of interaction between eIF3b and eIF3j in both organisms. However, analysis of the surface charge distribution of the putative RNA-binding $\beta$ sheet as well as the conservation of its RNA binding elements, suggested that in contrast to its human ortholog, yeast eIF3b-RRM could potentially bind oligonucleotides. Interaction studies 
with yeast total RNA extract confirmed the proposed RNA binding activity of yeast eIF3b-RRM. eIF3j/Hcr1, a loosely associated subunit of eIF3 has been previously shown to interact with Rli1, an iron-sulfur-cluster containing member of the super-family of ABC ATPases. In addition to translation initiation, Rli1 plays roles in ribosomal subunit maturation and transport of both ribosomal subunits into the cytoplasm. In chapter four, a novel function for Rlil in translation termination is presented. Rlil was shown to physically interact with the translation termination factors eRF1/Sup45 and eRF3/Sup35 in Saccharomyces cerevisiae. Genetic interactions were uncovered between a strain depleted for Rli1 and sup35-21 or sup45-2. Further, down regulation of the RLII expression was shown to cause defects in the recognition of a stop codon, as seen in mutants of other termination factors.

In chapter five, results obtained from different projects are discussed in a broad perspective. Furthermore, an expansion of the presented data is provided which should shed light on the herein presented results. The future perspective of the projects as well as suggestions for further experiments are presented in chapter six. 


\section{Chapter $1 \bullet$ Introduction}

The maintenance of cellular structure and functions requires an orchestrated synthesis of its building blocks together with a continuous flow of metabolic reactions. Proteins, as the most diverse and versatile macromolecules, play essential roles in all metabolic pathways and also contribute greatly to cellular integrity. Protein synthesis is therefore of vital importance for the cell. Hence, cells have developed complicated machineries and exploited many different factors to achieve accurate, yet rapid, protein synthesis.

In this chapter, first a brief introduction to protein synthesis and ribosome structure and function is presented which is followed by a closer look at the translation initiation step in eukaryotes. Later, different eukaryotic initiation factors are introduced briefly, with more emphasize on eIF3 which was the focus of this thesis. The elongation phase of translation was out of the scope of this project and is therefore only briefly mentioned. The last part of the introduction is dedicated to translation termination and ribosomal recycling in eukaryotes.

\subsection{A brief overview of protein synthesis}

Primary information about synthesis of proteins is hidden in the genome. Messenger RNA (mRNA) transcription is therefore the first step towards synthesis of proteins. In eukaryotes, most genes are transcribed as pre-mRNAs in which the coding sequences (exons) are interrupted by non-coding sequences (introns). Pre-mRNA transcription takes place in the nucleus where several post-transcriptional modifications occur to convert the pre-mRNA to a mature mRNA. These include addition of an unusual cap structure to the 5'-end of pre-mRNA, 3'-end cleavage and extension with a long poly-A tail and the precise removal of introns. In order to reach the cytosol which is where protein synthesis occurs, mRNAs have to pass through the nuclear envelope, in a process facilitated by nuclear export complexes. When in the cytoplasm, eukaryotic mRNAs, similar to archaeal and bacterial mRNAs, are recruited to the ribosome, a mega-Dalton machinery dedicated to protein synthesis.

Ribosomes are supramolecular ribonucleoprotein assemblies made up of mainly ribosomal RNAs (rRNAs) and several proteins. The functional ribosome is composed of two subunits: the small (30S in prokaryotes and 40S in eukaryotes) and the large (50S in prokaryotes and 60S in eukaryotes) subunits. Bacterial, archaeal and eukaryotic ribosomes share a common core responsible for mRNA decoding, peptide-bond formation and translocation of mRNA and tRNA 
by one codon at a time. Each subunit has specific features, while some features are common to both subunits. Among common features of the two subunits are three tRNA binding sites: the Asite accommodates the aminoacylated tRNA which will be added to the growing chain, the P-site which binds to the peptidyl-tRNA and the E-site which transiently accommodates deacylated tRNA exiting from the ribosome. The most important small subunit-specific feature is the mRNA binding channel and decoding center (DC), at which the base-pairing of codon-anticodon occurs. The peptidyl transferase center (PTC) is the most prominent feature of the large subunit triggering the formation of the peptide bond.

In all domains of life, protein synthesis follows the same concept. Translation initiates with recruitment of the mRNA to the small ribosomal subunit, binding of initiator tRNA to its P-site and deciphering of the start codon. However, mRNA recruitment follows different scenarios. In bacteria and archaea, a sequence complementarity between a region of mRNA close to the first AUG codon (Shine-Dalgarno sequence) and 3' end of the small subunit rRNA places the AUG start codon in the P-site. In contrast, eukaryotes exploit a much more complicated system of the mRNA recognition via its cap-structure by the help of dedicated proteins, named eukaryotic initiation factors (eIFs). Cap recognition is followed by the scanning of the mRNA in search for the start codon. Joining of the large ribosomal subunit is the signal to enter the elongation phase of protein synthesis which includes cycles of decoding of the A-site codon by acylated-tRNA followed by transfer of the nascent peptide chain from P-site tRNA on the A-site tRNA. Deacylated tRNA in the P-site has to exit the ribosome through the E-site and the A-site peptidyl tRNA replaces it in the P-site. This is accompanied by recruitment of a new acylated tRNA to the P-site with the help of elongation factors (EFs). During this process, mRNA and ribosome move by one codon relative to each other, resulting in the placement of a new codon in the P-site decoding center. When a stop codon enters the P-site, it is recognized by special proteins named release factors (RFs) which trigger the release of the newly synthesized peptide. The complex of ribosomal subunits, mRNA and deacylated tRNA will eventually be dissociated and recycled to start a new round of translation.

The control of protein synthesis occurs at multiple levels of initiation, elongation or termination. Initiation of translation is the most complex step in protein synthesis and therefore allows more intricate regulations. 


\subsection{Eukaryotic translation initiation}

Translation initiation in eukaryotes can be conceptually divided in two parts. The first part is the delivery of the initiator tRNA into the P-site of the $40 \mathrm{~S}$ subunit. The second part is the recognition of the mRNA, its recruitment to the $40 \mathrm{~S}$ subunit and subsequent scanning for the start codon.

\subsubsection{Flow of translation initiation}

In eukaryotes, translation initiation is a complex process, requiring orchestrated function of upto nine different initiation factors, eIFs. eIF2 in complex with GTP recognizes initiator methionyl tRNA to form a ternary complex (TC) and positions it in the small ribosomal P-site. This process is enhanced by several other initiation factors. In yeast, eIFs 3, 2, 5 and 1 are shown to bind to each other prior to the ribosomal binding to form a multi-factor complex (MFC) which binds to the $40 \mathrm{~S}$ ribosome to form $43 \mathrm{~S}$ pre-initiation complex (43S-PIC) (Figure 1.1).

The mRNA to be translated also has to be recognized by a set of initiation factors called eIF4F complex. eIF4F consists of the cap-binding protein eIF4E, the DEAD-box RNA helicase eIF4A and the scaffold protein eIF4G. eIF4A unwinds the secondary structures of the 5' UTR prior to the start codon in an ATP-dependent manner. To exert its function, eIF4A requires another protein, eIF4B. It was shown that eIF4G interacts with eIF3 in mammalian cells, establishing the chain of interactions of cap-eIF4E-eIF4G-eIF3, which recruits 43S-PIC to the 5 ' end of the capped mRNA to form the 48S-PIC.

eIF1 binds near the P-site to the interface between the platform and initiator methionyl tRNA (Met-tRNA ${ }_{\text {i) }}^{\text {Met }}$ eIF1A's structured domain resides in the A-site whereas its unstructured termini extend into the P-site. Cryoelectron microscopy reconstructions of the yeast $40 \mathrm{~S}$ ribosomal subunit in apo or eIF1-eIF1A bound states have revealed that binding of eIF1 and eIF1A to the small ribosomal subunit induces conformational changes leading to the opening of the mRNA entry channel latch and establishment of a new head-body connection on the solvent side (Passmore et al. 2007). This "open" conformation makes the ribosome competent for scanning. The scanning ribosome unwinds the secondary structures of the mRNA and moves along it.

eIF1 assures the fidelity of the translation initiation by discriminating against non-AUG start codons, AUGs within a poor context or within eight nucleotides from 5' end of the mRNA. This is achieved by antagonizing conformational changes that occur in ribosomal complexes upon 
codon-anticodon base pairing during the formation of the 48S PIC (Pestova and Kolupaeva 2002; Pisarev et al. 2006). eIF1A's C-terminal domain also contributes to the accuracy of start codon selection by promoting the "open" conformation of the scanning ribosome. Proper AUGcodon recognition induces conformational changes in the ribosome which on one hand displaces eIF1 from a near P-site position and on the other hand tightens the eIF1A-40S interaction. Both of these events lead to a "close" conformation of the 48S-PIC. In the scanning-competent $48 \mathrm{~S}$ PIC, eIF1 represses premature hydrolysis of eIF2-bound GTP and subsequent $\mathrm{P}_{\mathrm{i}}$ release. However, its displacement upon start-codon selection allows eIF5-mediated GTP hydrolysis by eIF2 and subsequent commitment of the ribosome to the start codon. In order to participate in another cycle of initiation, eIF2 has to be recharged with GTP which is facilitated by its guanine nucleotide exchange factor (GEF) eIF2B.

Dissociation of the factors and the $60 \mathrm{~S}$ subunit joining is mediated by the ribosome-dependent GTPase eIF5B, a homologue of bacterial IF2. The interaction of the C-termini of eIF5B and eIF1A stimulates subunit joining and GTP hydrolysis of eIF5B, which in turn releases eIF5B form $80 \mathrm{~S}$. Recruitment of the eIF5B also triggers the release of eIF1A that opens up the ribosomal A-site. Since eIF3 and eIF4G occupy the solvent exposed side of the 40S subunit, their dissociation might be delayed. Such a delay could in turn assist the re-initiation on short upstream open reading frames (for comprehensive reviews see Sonenberg and Hinnebusch, 2009 and Jakson et al. 2010 ).

\subsubsection{The role of initiation factors}

\subsubsection{1. mRNA recognition and eIF4F}

The $5^{\prime} \mathrm{m}^{7} \mathrm{G}$ cap of mRNA is recognized by the eIF4F complex, consisting of the cap-binding protein eIF4E, the DEAD-box RNA helicase eIF4A and the scaffold protein eIF4G. eIF4A unwinds the secondary structures of the 5' UTR prior to the start codon. To exert its function, eIF4A requires another protein, eIF4B. Mammalian eIF4G consists of a long N-terminal region followed by three HEAT repeat domains named HEAT-1, 2 and 3. The N-terminal region of eIF4G harbors the interaction site for the poly-A binding protein (PABP) at a position far from the HEAT repeats, and also interacts with the cap-binding protein eIF4E. The first two HEAT repeats interact with eIF4A, with HEAT-1 stimulating its helicase activity and HEAT-2 having a modulatory role. HEAT-1 makes extra contacts with eIF3 as well as with mRNA (Marintchev and Wagner 2004). 


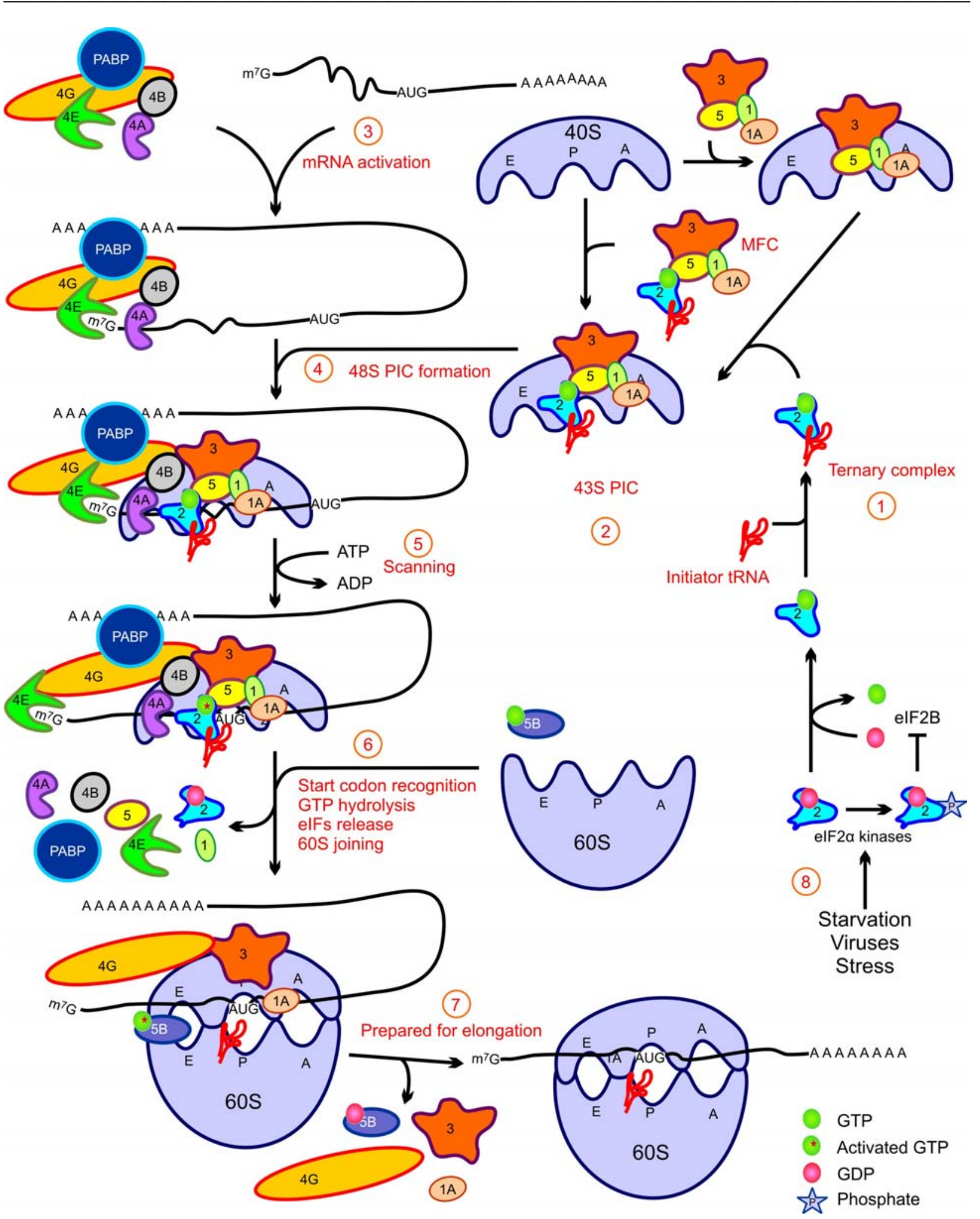

Figure.1. 1. Schematic view of eukaryotic cap-dependant translation initiation. Each factor is shown as a colored figure. Formation of the ternary complex from elF2, Met-tRNA ${ }_{i}^{\mathrm{Met}}$ is the first step of translation initiation (1). Ternary complex will join the $40 \mathrm{~S}$ subunit either alone or through multi factor complex (MFC) to form the 43S PIC (2). The mRNA is activated by elF4F complex (3). The activated mRNA is then recruited to the $43 \mathrm{~S}$ PIC to form $48 \mathrm{~S}$ PIC (4). 48S PIC scans the mRNA in an ATP dependent manner in search for the start codon (5). Upon start codon selection and the hydrolysis of elF2-bound GTP, the 40S subunit is committed to the start codon, followed by dissociation of most of the initiation factors and 
joining of the 60 S subunit (6). The hydrolysis of elF5B-bound GTP triggers the release of elF3, elF4G and elF1A, signaling the beginning of the elongation step (7). Under stress conditions, different kinases phosphorylated elF $2 \alpha$, converting it to the inhibitor of its GEF, elF2B (8).

eIF4A is a member of the DEAD-box family of helicases, containing only two RecA-like domains involved in binding RNA and ATP. However, eIF4A requires accessory proteins eIF4H/B and eIF4G for its optimal activity. In the "closed" ATP-bound state, two domains of eIF4A form a contiguous mRNA binding surface with the ATP binding site lying at the interface between two domains (Andersen et al. 2006). eIF4H and eIF4B are RNA binding proteins containing an RNA recognition motif (RRM) and stimulate the helicase activity of eIF4A. They are homologous to each other over the whole eIF4H, however eIF4B harbors an extra RRM near its C-terminus.

Recently, a study using NMR, site directed mutagenesis and thermodynamic analysis shed light on the topology of eIF4A/4G/4H and showed the importance of a dynamic network of multiple weak yet specific interactions in the function of this complex (Marintchev et al. 2009). According to this study, in the absence of ADP or ATP, eIF4A binds to eIF4G HEAT-2 much tighter than to HEAT1, stabilizing the complex in the open conformation. ATP binds cooperatively with eIF4G HEAT-1 and mRNA and anticooperatively with HEAT-2. The fact that binding of the RNA competes with HEAT-2 suggests that RNA binding to eIF4A would displace HEAT-2 and shifts the equilibrium towards the ATP-bound "close" state. ATP hydrolysis and subsequent ADP release would decrease the affinity of eIF4A for RNA and eIF4G HEAT-1 and increase the affinity for HEAT-2, driving the equilibrium towards the "open" state. Interaction of eIF4A with eIF4H is also ATP dependent, with formation of a tight complex with eIF4H in the presence of ATP followed by the weakening of the complex upon hydrolysis of ATP and subsequent release of ADP. In analogy to the available structures of eIF4A homologs in complex with RNA, Wagner and colleagues suggested the N- and C-terminal domains of eIF4A to be oriented towards the 3' and 5' ends of the mRNA, respectively. Interestingly, both eIF4G HEAT1 and eIF4H can contact mRNA. Mapping the interaction site of mRNA and eIF4H-RRM on eIF4A by NMR perturbation analysis suggested that the RRM domain of eIF4H can contact the mRNA immediately 5' from eIF4A. This would place the RRM domain behind the helicase with respect to the direction of translocation. Taken together, a model was suggested in which eIF4G HEAT-1 tethers eIF4A to mRNA, whereas binding of eIF4H to single stranded mRNA behind eIF4 prevents reannealing and confers processive unidirectional translocation of eIF4A. 
Most of eukaryotic mRNAs are recognized through the specific interactions between their 5' $\mathrm{m}^{7} \mathrm{G}$ cap and the eIF4E subunit of eIF4F complex. However, some mRNAs, particularly many viral mRNAs, bypass this process by using specialized sequences, called internal ribosome entry sites (IRESs). Some IRESs, such as those of picornaviruses, only circumvent the need for eIF4E but need all other initiation factors. The IRES of hepatitic $\mathrm{C}$ virus dispenses eIF4F completely, bu requires eIF3 and either eIF2/eIF5 or eIF5B. In extreme case, the IRES element perform the function of all eIFs, such as the IRES of cricket paralysis virus (reviewed in Cullen 2009).

The recruited mRNA has to be scanned by the ribosome in search for the proper start codon. Scanning involves intricate interaction between ribosome, mRNA and different initiation factors, including eIF1 and eIF1A.

\subsubsection{Roles of eIF1, eIF1A and eIF5B in scanning, start codon selection and subunit joining}

The binding of eIF1 and eIF1A to the 40S ribosomal subunit is thermodynamically coupled and occurs even in the absence of other initiation factors (Maag and Lorsch 2003). The structure of eIF1A resembles prokaryotic IF1 and is composed of an OB domain and a helical domain, which is packed against two $\beta$-strands. These domains are flanked by unstructured regions at both termini (Figure 1.2 B-C) (Battiste et al 2000).

Determination of the position of eIF1A on the $40 \mathrm{~S}$ ribosomal subunit by hydroxyl radical probing has shown the $\mathrm{OB}$ domain to bind in the A-site whereas the helical domain bridges over the mRNA channel and contacts the head domain. The N- and C-terminal tails reach into the Psite (Yu et al. 2009). Two sequences at the C-terminal tail (CTT) of eIF1A, namely $\underline{\text { Scanning }}$ Enhancer 1 and 2 (SE1 and SE2), are found to stimulate the recruitment of the TC on one hand and block the initiation at non-AUG codons on the other hand. A second pair of sequences termed Scanning Inhibitor 1 and 2 (SI1 and SI2) is located at the N-terminal tail (NTT) and the helical domain of eIF1A, respectively. These two sequences were found to play the opposite role to $\mathrm{SE}$ sequences by impeding the TC recruitment and increasing the rate of initiation at UUG codon in SE mutants (Saini et al 2010). The C-terminal domain of eIF1A is involved in an energetic interaction with eIF5 on the ribosome in the presence of AUG codon in the P-site. This interaction shifts the equilibrium between open and closed conformations of 43S PIC towards the closed conformation (Maag et al 2006). Maag and colleagues propose that the preferential formation of the closed conformation may play a role in the timing of eIF5B recruitment to the ribosome by releasing the eIF1A-CTD from interaction with eIF5 and allowing it to establish a 
new connection with eIF5B (Maag et al 2006). Location of the AUG codon in the 43S PIC triggers fast conformational changes which are followed by a slower decrease in the affinity of the complex for eIF1 (Maag et al 2005). In addition, a mutation in eIF1 (sui1-1), which shows an elevated rate of non-AUG codon recognition, has been shown to reduce binding of eIF1 to the preinitiation complex in vivo (Valasek et al. 2004). All together, these observations suggest that eIF1 is a negative regulator of GTP hydrolysis by eIF2 and its release upon recognition of the start codon triggers further steps in initiation. Interestingly, kinetic dissection of the start codon selection suggested that the $P_{i}$ release from eIF2, and not the GTP hydrolysis itself, is the step controlled by start codon selection (Algire et al 2005). In this view, a structural rearrangement takes place within the 43S PIC, which facilitates eIF5-mediated GTP hydrolysis even in the absence of mRNA. However, the release of inorganic phosphate from eIF2 is dependant on the recognition of the cognate start codon and the subsequent release or displacement of eIF1 (Algire et al 2005).

The solution structure of eIF1 shows a compact core made up of a globular $\alpha / \beta$ domain with a long highly disordered tail at the N-terminus (Figure 1.2 A; Fletcher et al. 1999; Reibarkh et al. 2008). Hydroxyl radical probing of the eIF1 places it on the interface surface of the platform of the $40 \mathrm{~S}$ subunit in the proximity of the ribosomal P-site (Lomakin et al. 2003). The position of eIF1 on the 40S subunit is similar to the position of IF3 on the prokaryotic $30 \mathrm{~S}$ ribosome which also participates in the initiation-codon selection (Dallas and Noller 2001; Tedin et al. 1999). $48 \mathrm{~S}$ complexes assembled on AUG codons with an unfavorable context in the absence of eIF1 would dissociate upon its addition (Pestova and Kolupaeva 2002). Therefore, positioning of eIF1 near the P-site might increases its influence on selection of the initiation codons within the context of the mRNA.

Kinetic and thermodynamic analysis of the start codon-anticodon selection has revealed that base changes in the first position of the start codon are tolerated for TC binding, whereas any changes in the second or third position of the start codon reduce the binding and impair the initiation process both in vitro and in vivo. Studying the dissociation constant $\left(k_{o f f}\right)$ of TC in the presence of cognate versus near-cognate start codon does not show a significant difference. However, the association constant $\left(k_{o n}\right)$ of the TC is 1000 -fold higher in the presence of the cognate start codon in comparison with the near cognates. Surprisingly, restoring the codonanticodon base pairing by mutating both mRNA start codon and $\mathrm{RNA}_{\mathrm{i}}{ }^{\mathrm{Met}}$ anticodon restores the binding to the wild-type values. This suggests that the formation of three base pairs, regardless of their identities, governs the stability of TC binding (Kolitz et al. 2009). 
A

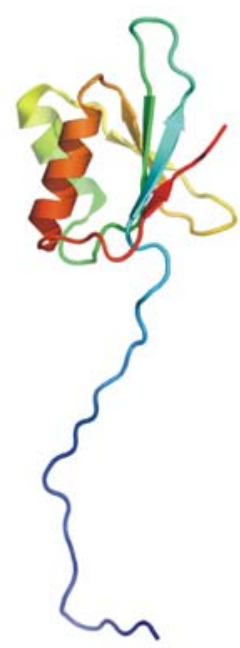

B

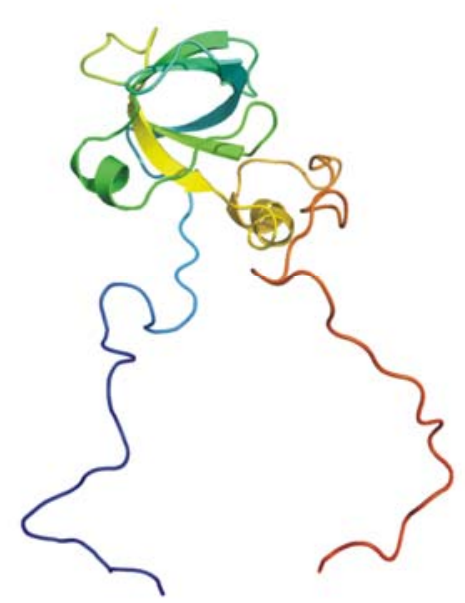

C

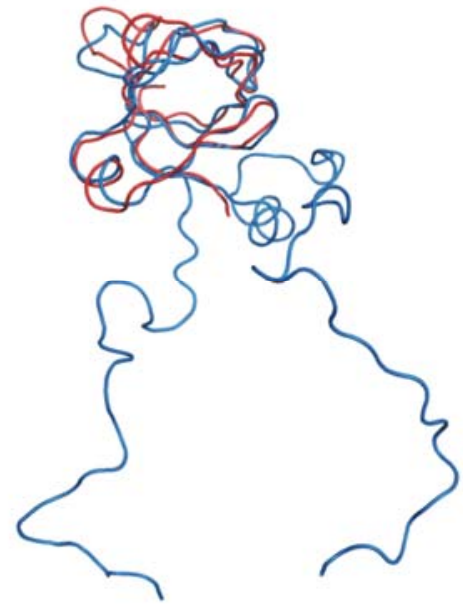

Figure.1. 2. Structures of elF1 and elF1A. (A) Solution structure of yeast elF1 (PDB code 2OGH) shows a compact $\alpha / \beta$ domain preceeded by a long unstructured $N$-terminal tail. (B) Solution structure of human eIF1A (PDB code 1D7Q) reveals an OB domain and a helical domain which is packed against two strands, flanked by two unstructured tails and both termini. (C) Structural comparison of human elF1 (blue) and IF1 from Mycobacterium tuberculosis (red, PDB code 3140) reveals that the globular core of both proteins is highly similar.

An NMR study of the interaction between eIF1A and eIF5B has determined the DIDDI motif at the C-terminus of eIF1A to be the interaction partner of the eIF5B C-terminal helical binding pocket (Marintchev et al 2003). This interaction in the context of initiation dependant subunit joining is required for the full GTPase activity of eIF5B (Acker et al 2006). Mutation of either isoleucine residues in DIDDI sequence reduces both the rate constant of GTP hydrolysis by eIF5B and its ability to facilitate subunit joining, whereas changing the aspartate residues has no effect (Acker et al 2006). eIF5B is located in the intersubunit cleft of the 80S ribosome (Unbehaun et al. 2007). The interaction between eIF1A and eIF5B is required for the stimulation of subunit joining in the process of translation initiation, as disruption of this interaction, e.g. by deleting the C-terminal DIDDI sequence of eIF1A, reduces the rate of subunit joining (Acker et al. 2009). GTP hydrolysis by eIF5B on one hand results in the reduction of its affinity for $80 \mathrm{~S}$ and its subsequent dissociation (Pestova et al. 2000) and on the other hand affects the dissociation of eIF1A form the initiating ribosome (Fringer et al. 2007). Kinetic dissection of the 80 S complex formation has shown that replacing GTP with non-hydrolysable GDPNP in the wild-type eIF5B does not affect the rate of subunit joining. Dissociation of eIF1A from 80S IC is also accelerated by GTP hydrolysis by eIF5B. It is worth mentioning that the rate of dissociation of eIF1A from an 80S IC formed with eIF5B-GDPNP is higher than the dissociation rate of 
eIF5B-GDPNP itself. This implies that, although GTP hydrolysis by eIF5B accelerates the dissociation of eIF1A, eIF1A is not fully trapped in the complex by eIF5B-GDPNP and is able to leave the complex prior to eIF5B dissociation (Acker et al. 2009).

\subsubsection{3. eIF2 and initiator tRNA delivery to the $40 \mathrm{~S}$}

eIF2 is a heterotrimeric protein, made up of three subunits; $\alpha, \beta$ and $\gamma$, and is responsible for the delivery of the initiator methionyl tRNA into the small ribosomal P-site. The genes coding for these proteins are essential for yeast cell viability (Cigan et al. 1989; Donahue and Cigan 1988; Hannig et al. 1993). eIF2 subunits have archaeal orthologs, named aIF2 $\alpha, \beta$ and $\gamma$ (Woese 1998) but are missing among bacteria. The $\alpha$ subunit in both domains is composed of three parts: an Nterminal $\beta$-barrel followed by an $\alpha$-helical and an $\alpha-\beta$ domain. Domains 1 and 2 form a rigid body, which is linked to the mobile third domain (Dhaliwal and Hoffman 2003; Ito, Marintchev, and Wagner 2004; Yatime et al. 2005). The C-terminus of eukaryotic eIF2 $\alpha$ harbors an acidic extension (Figure 1.3 A). aIF2 $\beta$ is built up of an N-terminal $\alpha$-helix followed by a central $\alpha-\beta$ domain and a zinc binding domain (ZBD) at the C-terminus (Cho and Hoffman 2002; Gutirrez, Osborne, and Siddiqui 2004). The eukaryotic eIF2 $\beta$ is believed to have the same domain architecture as archaeal aIF2 $\beta$ with addition of two extensions at both termini (Figure $1.3 \mathrm{~B}$ ). The N-terminal extension harbors three lysine-rich boxes which are involved in interactions with acidic/aromatic boxes (AA boxes) at the C-termini of eIF5 and eIF2B $\varepsilon$ (Asano et al. 1999). No specific role has so far been assigned to the C-terminal extension of eIF2 $\beta$. e/aIF2 $\gamma$ is the central component of e/aIF2 complex which interacts with the other two subunits (Pedullà et al. 2005). aIF $2 \gamma$ has a G-domain (domain I) followed by two $\beta$-barrel domains (domains II and III). Eukaryotic eIF $2 \gamma$ is homologous to aIF2 $\gamma$ with the exception that it has an N-terminal tail of varying length (Figure $1.3 \mathrm{C}$ ). In budding yeast this tail is not essential. However, mutations within it are responsible for a slow growth phenotype (Erickson et al. 2001). The G-domain contains all features of G-proteins, including the guanine nucleotide binding pocket, switch 1 and switch 2 regions (for a review see Schmitt, Naveau and Mechulam 2010 and references therein). This domain architecture resembles that of elongation factor EF-Tu or eEF1A (Andersen et al. 2001; Berchtold et al. 1993). Most of the crystal structures of archaeal aIF2 $\gamma$ are obtained either with GDP or non-hydrolysable analogs of GTP. These structures have shown the "closed" conformation of the domains together with the "off" state of the switch regions, which cannot explain the GTP-dependent tRNA binding of e/aIF2 (Schmitt, Blanquet, and Mechulam 2002; Nikonov et al. 2007). The low affinity of archaeal aIF2 $\gamma$ for Met-tRNA ${ }_{i}^{\text {Met }}$ is increased upon interaction with aIF2 $\alpha$, whereas aIF2 $\beta$ has no effect on that (Yatime et al. 2004). In 
contrast, the eukaryotic eIF2 $\alpha$ was shown to have little impact on the affinity of eIF2 $\gamma$ for the initiator tRNA, whereas eIF2 $\beta$ enhances this binding (Nika, Rippel, and Hannig 2001).

A

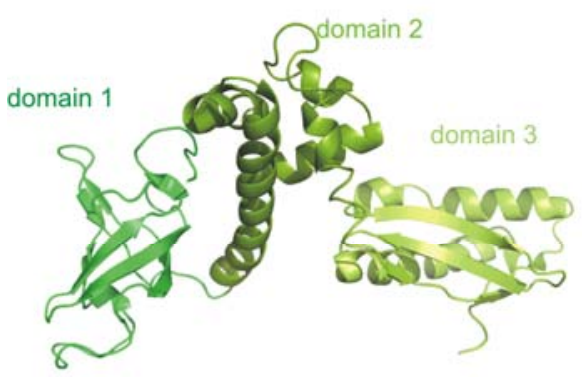

B
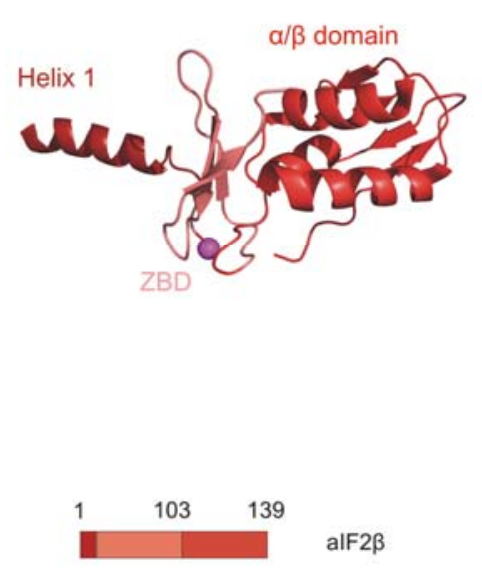

\begin{tabular}{llll}
1 & 125 & 236 & $272-285$ \\
\hline & & elF2 $\beta$
\end{tabular}
C
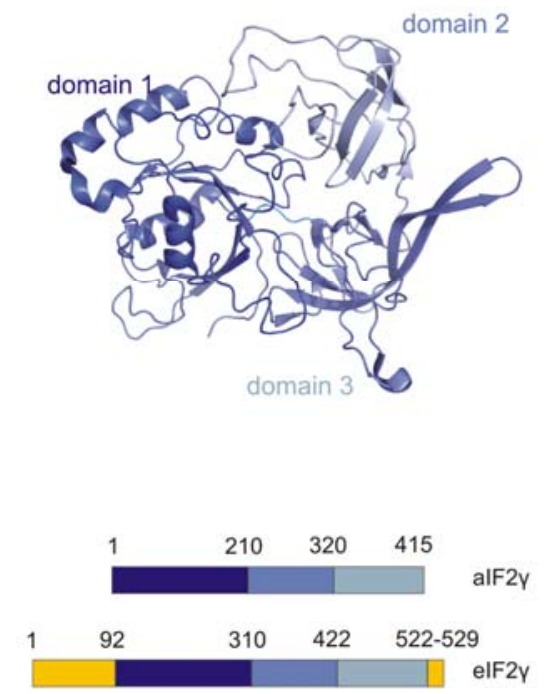

D

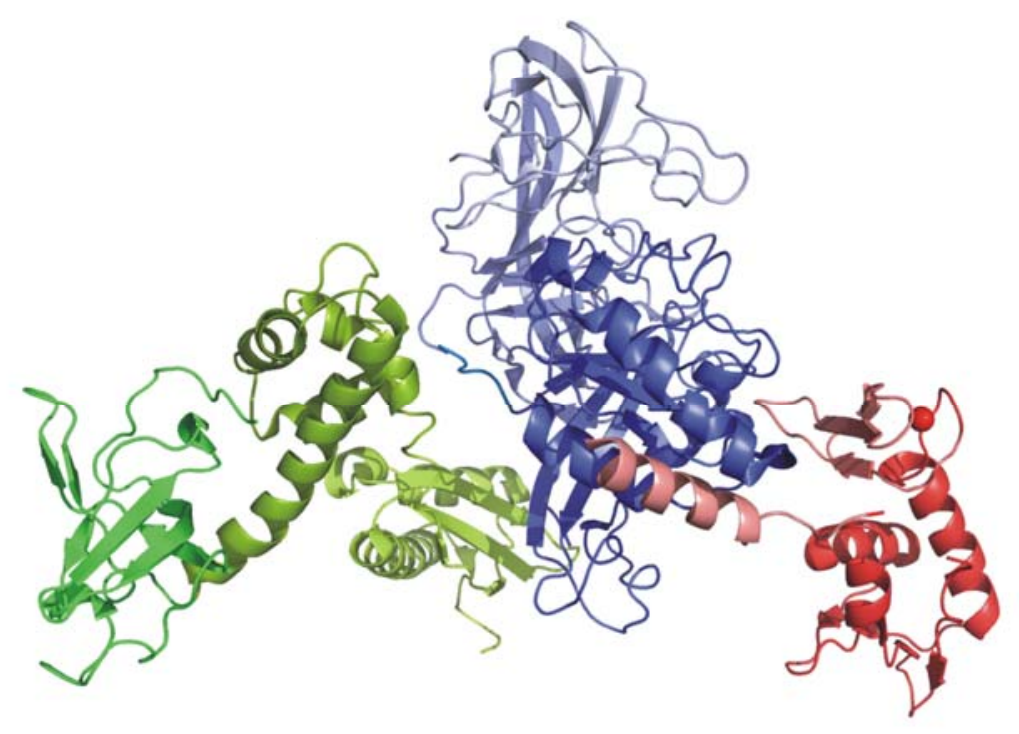

Figure.1. 3. Structure of archaeal alF2. (A) alF2 $\alpha,(B)$ alF2 $\beta$ and (C) alF2 $\gamma$ from Sulfolobus solfataricus as seen in the alF2 complex (PDB code 3CW2). In each case, the domain architecture of archaeal and eukaryotic versions are shown below the structure, with colors representing the corresponding domain in the structure. The eukaryotic-specific expansions are colored yellow. (D) The structure of intact alF2 complex from Sulfolobus solfataricus (PDB code 3CW2) shows the binding of $\alpha$ and $\beta$ subunits to the opposite sides of alF2 $\gamma$.

The crystal structure of Sulfolobus solfataricus (Ss-) aIF2 $\alpha$ y complex in presence of Gpp(NH)p$\mathrm{Mg}^{2+}$ has shown the switch regions of the $\gamma$ subunit to be in the "on" conformation (Yatime et al. 2006), similar to the structure of EF-Tu in complex with Phe-tRNA ${ }^{\text {Phe }}$ and a non-hydrolysable 
GTP analog (Nissen et al. 1993). Docking of the tRNA on the aIF2 surface suggested that the altered position of the switch 1 in the "on" state opens a channel between domains I and II of the $\gamma$ subunit. This channel accommodates the methionine moiety of the tRNA. In this model, the last base of the tRNA (A76) resides in a pocket at the surface of domain II. Thermodynamic coupling between the binding of GTP and Met-tRNA ${ }_{i}{ }^{\text {Met }}$ to eIF2, not observed with unacylated tRNA $_{i}{ }^{\text {Met }}$ or between GDP and Met-tRNA ${ }_{i}^{\text {Met }}$, indicates that there is a positive contact between GTP-bound eIF2 and the methionyl moiety on the tRNA. This interaction is suggested to contribute to the discrimination between acylated and unacylated initiator tRNA. Induction of the "on" conformation of the switch regions of aIF $2 \gamma$ by GTP analogs probably accounts for the observed dependence of the Met-tRNA ${ }_{i}^{\text {Met }}$ binding affinity on the GTP bound state of the protein. Interestingly, the affinity of eIF2 for unacylated tRNA is the same in both GDP and GTP bound states, and is similar to the affinity of GDP-bound eIF2 for Met-tRNA ${ }_{i}^{\text {Met }}$. This further indicates the additive contact between eIF2 and methionyl moiety in the GTP bound state (Kapp and Lorsch 2004a). In the above-mentioned model of aIF2 $\alpha \gamma$-tRNA no direct contact is observed between $\alpha$ subunit and the tRNA. However, an indirect role of aIF2 $\alpha$ in helping aIF $2 \gamma$ to maintain the switches in the "on" conformation cannot be ruled out (Schmitt, Naveau and Mechulam 2010). However, structure determination of eukaryotic eIF2 is needed to shed light on the apparent dependence of the tRNA binding of eIF $2 \gamma$ on the presence of the $\beta$ subunit in eukaryotes.

\subsubsection{4. eIF5, the GTPase activating protein}

eIF5 consists of two distinct domains; the N-terminal domain, which fulfills the GAP function of eIF5 and the C-terminal domain which interacts with other initiation factors. NMR solution structure of eIF5-NTD shows that it is composed of two sub-domains. The most N-terminal domain surprisingly resembles the structural organization of eIF1 in spite of a lack of sequence homology between them. This part harbors the putative arginine finger flanking by aromatic side chains in a very flexible loop which is believed to get ordered upon interaction with eIF $2 \gamma$. The more C-terminal sub-domain contains a zinc finger (Figure 1.4). Structural homology between eIF5-NTD and the archaeal aIF2 $\beta$ has led to the suggestion of a common fold for some initiation factors called "eIF125 fold" (Figure 1.5; Conte et al. 2006).

The C-terminal domain of eIF5 mainly serves to interact with other initiation factors required for 

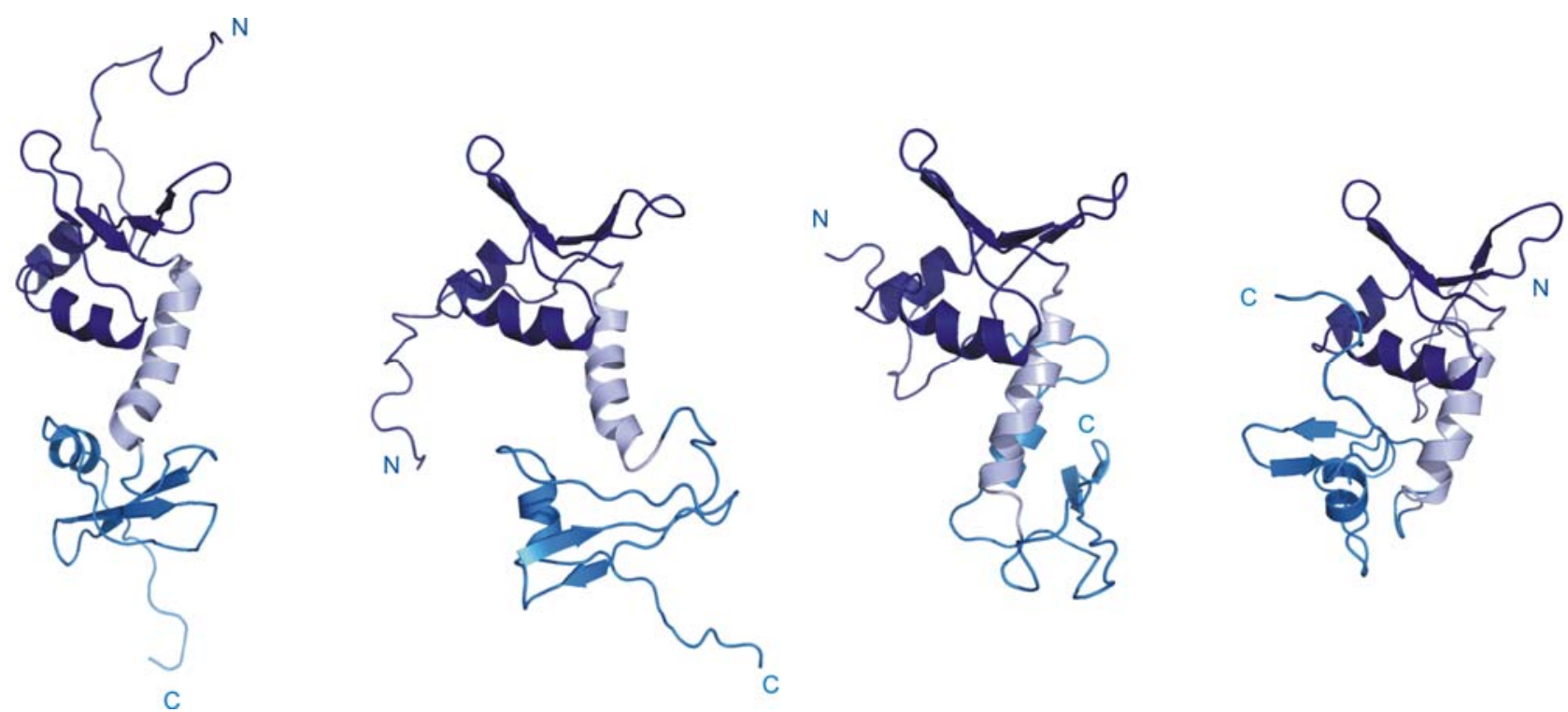

Figure.1. 4. High flexibility of the $\mathbf{N}$-terminal domain of human elF5. The $\mathrm{N}$-terminal $\alpha / \beta$ subdomain depicted in dark blue is connected via an $\alpha$-helix (light blue) to the C-terminal subdomain (marine blue) containing the zinc finger. The four structures are different conformations of the same protein in solution extracted from the NMR structure (PDB code $2 \mathrm{G} 2 \mathrm{~K}$ ) and aligned based on the N-terminal subdomain.

A

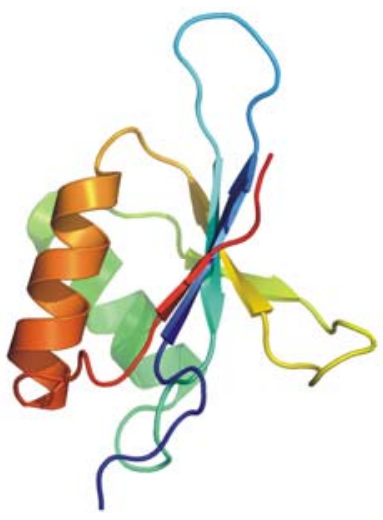

B

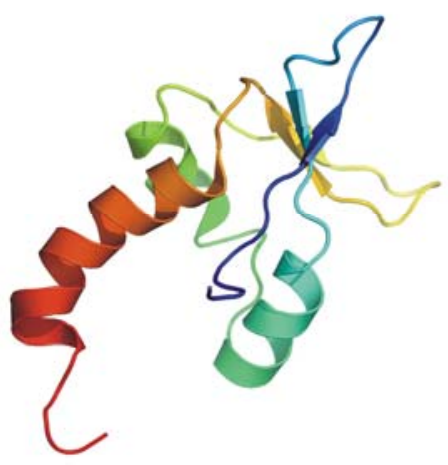

C

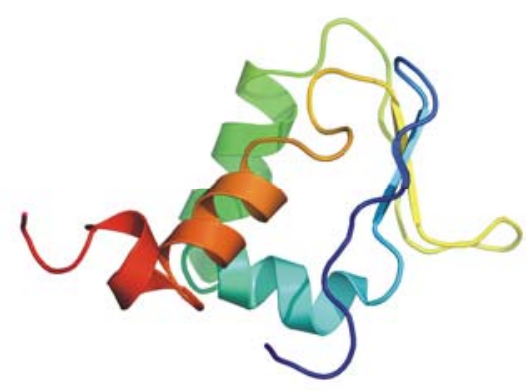

Figure.1. 5. Structural similarity between elF1, elF5-NTD and e/aIF2 $\beta$. (A) NMR structure of yeast elF1 (PDB code $2 \mathrm{OGH}$ ). The $\mathrm{N}$-terminal disordered tail is removed for the sake of clarity. (B) The N-terminal subdomain of human elF5-NTD (PDB code 2G2K). (C) The middle domain of archaeal alF2 $\beta$ containing the $\alpha / \beta$ domain (PDB code $3 C W 2$ ).

the incorporation of eIF5 into MFC. Mutations in this domain reduce the recruitment of both mRNA and Met-tRNA ${ }_{i}^{\text {Met }}$ to the ribosome which might be due to the disruption of the interactions of eIF5 with eIF4G and eIF2 $\beta$, respectively. Simultaneous interaction of eIF5-CTD with eIF4F (via eIF4G) and eIF3 (via Nip1) may enhance association of eIF4F with eIF3 and promote mRNA binding to the ribosome (Asano et al. 2001). The C-terminal domain of eIF5 
possesses an atypical HEAT motif similar to the C-terminus of the catalytic $(\varepsilon)$ subunit of eIF2B (Figure 1.6), with three distinct charged areas on the surface (Wei et al. 2006; Bieniossek et al. 2006). The first area is made up of two acidic/aromatic residue-rich regions (AA-boxes) which are located in a negatively charged patch on the surface of the protein. Two AA boxes are conserved between the $\mathrm{C}$-termini of eIF5 and eIF2B $\varepsilon$, and are required for their interactions with three lysine-rich regions (K boxes) of eIF2 $\beta$ (Asano et al. 1999). Interestingly, eIF5 and eIF2B $\varepsilon$ are absent in archaea and archaeal aIF2 $\beta$ lacks the K-boxes. The binding of eIF5-CTD to eIF4G competes with its binding to eIF2 $\beta$ (Asano et al. 2001). Therefore Asano and colleagues suggested that the binding site for eIF4G overlaps with that of eIF2 $\beta$ on eIF5-CTD (Yamamoto et al. 2005). The second charged area on the surface of eIF5-CTD is a positively charged region composed of several lysine residues. This area is proposed to interact with eIF1 and the Nterminal domain of Nip1 (Asano et al. 2000; Yamamoto et al. 2005). The third charged area of eIF5-CTD is another acidic patch is located almost on the opposite side of the positively charged area and is suggested to interact with other K-boxes on eIF2 $\beta$ (Wei et al. 2006).

Recently, the linker region (LR) between the N- and C-termini of eIF5 was shown to interact directly with eIF2 and exert GDI activity, independent of the GAP function of the N-terminal domain. Despite the lethality of the mutations affecting the GAP function, mutations in the linker region, which impair the GDI function in vitro are not lethal in vivo. This proposes that the GAP, and not the GDI, activity is the essential function of eIF5. The eIF5 GDI function is suggested to be a novel component of the inhibitory eIF2-phosphorylation pathway which restricts the recycling of the eIF2 to ternary complex. Therefore it acts in addition to the wellknown inhibition of the GEF activity of the eIF2B (Jennings and Pavitt 2010).

A

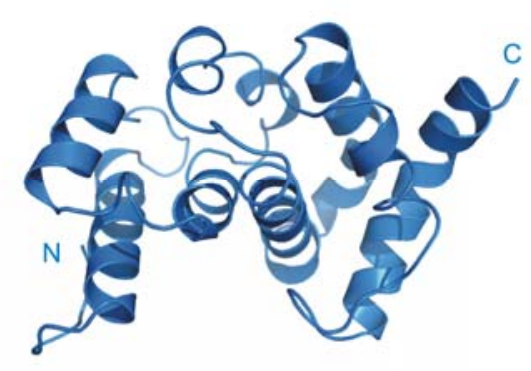

B

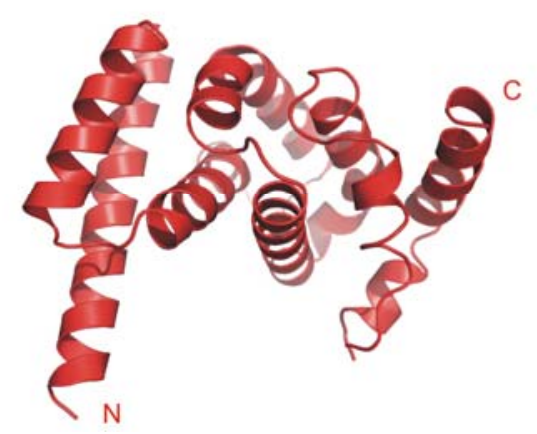

C

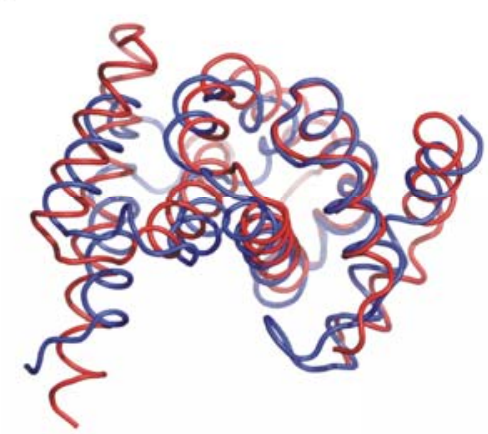

Figure.1. 6. Structural comparison of the C-terminal domains of elF5 and elF2B $\varepsilon$. (A) Yeast elF5-CTD is highly $\alpha$-helical, creating several surfaces for interaction with different proteins (PDB code 2FUL) (B) Human elF2B $\varepsilon$-CTD is also $\alpha$-helical, with a long $\alpha$-helix at the $N$-terminus in comparison with elF5-CTD (PDB code 3JUI) (C) Structure based alignment of the c-termini of yeast elF5 and human elF2B $\varepsilon$ shows high structural similarity . 


\subsubsection{5. eIF3; a versatile scaffold}

eIF3 is the largest initiation factor with five stoichiometric subunits in budding yeast and twelve in human. In spite of its complexity, human eIF3 shares a common core with yeast eIF3 composed of eIF3a, b, c, g and i. The sixth subunit of yeast eIF3 is eIF3j, a loosely associated member of the eIF3 complex, which also has functions in ribosomal biogenesis. Hinnebusch and colleagues have shown that eIF3 makes critical interactions with other initiation factors (Figure 1.7). Through its eIF3a/Tif32 and eIF3c/Nip1 subunits, eIF3 binds to the N-terminal domain of eIF2 $\beta$ and the C-terminal domain of eIF5, respectively (Valásek, Nielsen, and Hinnebusch 2002; Valásek et al. 2003; Nielsen et al. 2004). Yeast eIF3, eIF1, eIF5 and the ternary complex can be isolated in a multifactor complex (MFC) free of the 40S ribosomal subunit (Asano et al. 2000) suggesting their cooperative binding to the 40S subunit (Hinnebusch 2006).

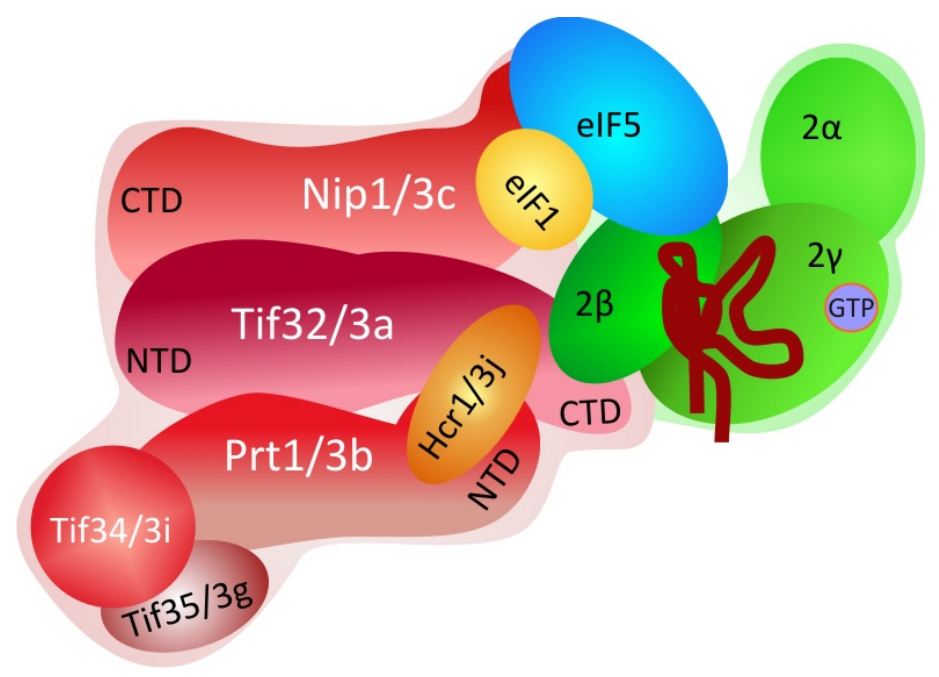

Figure.1. 7. The schematic model of yeast multi-factor complex. This model is based on binary interactions between isolated recombinant subunits and the affinity purification of sub-complexes. elF3 subunits are colored red, purple or orange. elF2 is depicted in green. elF5 and elF1 are shown in blue and yellow, respectively. tRNA is depicted as a twisted dark red line of elF2.

\subsection{The role of eIF3 in $\mathrm{mRNA}$ recruitment and scanning}

eIF3 functions in mRNA recruitment and scanning. Depletion of eIF3a and eIF3b in yeast degron mutants as well as the Prt1-1 mutation in yeast eIF3b impair mRNA binding to the $40 \mathrm{~S}$ subunit (Phan et al. 2001; Jivotovskaya et al. 2006). Direct interaction of mammalian eIF3 with eIF4G, the scaffolding subunit of eIF4F, is implicated in mRNA recruitment to the ribosome 
(Korneeva et al. 2000). However, the eIF3-interacting domain is not conserved in yeast eIF4G and there has not been any reported interaction between yeast eIF3 and eIF4G. Depletion of eIF2 or eIF3 impairs mRNA binding to the free 40S subunit, while the depletion of eIF4G from yeast leads to the accumulation, rather than the depletion, of mRNAs in the native yeast 48S PIC (Jivotovskaya et al. 2006). Thus, eIF3 can promote the recruitment of some mRNAs independently of eIF4G in yeast. In budding yeast, eIF5-CTD binds to eIF4G and eIF3, and thus could form a bridge between them to promote mRNA recruitment (Asano et al. 2001). Mammalian and yeast eIF4B are reported to interact with eIF3a and eIF3g, respectively (Methot et al. 1996; Vornlocher et al. 1999). This interaction may provide another connection between eIF3 and eIF4. Some of the eIF3's subunits have RNA binding activity and can be crosslinked to mRNA in 48S PIC and in mRNA-eIF3 complexes in vitro (Asano et al. 1997a; Asano et al. 1997b; Hinnebusch 2006; Kolupaeva et al. 2005; Unbehaun et al. 2004; Khoshnevis, Neumann, and Ficner 2010). Therefore, in addition to serve as an adaptor between eIF4F and the ribosome, eIF3 may bind to mRNA directly. Mutations in yeast eIF3c-NTD, which disrupt its interactions with eIF5 and eIF1 facilitate translation initiation at UUG codon in vivo. Such a phenotype suggests that eIF3 is also involved in scanning and AUG recognition after mRNA recruitment (Valásek et al. 2004). eIF3b is believed to be the major scaffolding subunit of eIF3 since it interacts with $3 \mathrm{a}, 3 \mathrm{c}, 3 \mathrm{~g}, 3 \mathrm{i}$ and $3 \mathrm{j}$ both in yeast and in mammals. Mutation of the ribonucleoprotein (RNP) 1 motif within eIF3b-RRM increases leaky scanning, proposing a role in AUG recognition. Strong leaky scanning phenotype is also observed if the N-terminal domain of eIF $3 \mathrm{j}$ is deleted which is suppressible by overexpression of eIF1A. This strongly suggests that eIF3j stays bound to scanning ribosome even after mRNA recruitment.

\subsection{Ribosome binding by eIF3}

Yeast eIF3 binds the ribosome through direct interactions between the N-terminal domain of eIF3a/TIF32 with the ribosomal protein RPS0A. The C-terminal domain of eIF3a can also specifically bind to a fragment of $18 \mathrm{~S}$ rRNA encompassing helices 16-18. These interactions propose that eIF3 associates with the solvent-exposed side of the $40 \mathrm{~S}$ subunit, in the vicinity of the mRNA exit channel (Valásek et al. 2003). In accordance with the yeast model, cryoelectron microscopy of human eIF3 in combination with modeling has proposed a five-lobed particle which together with eIF4G occupies the solvent exposed surface of 40S ribosome (Siridechadilok et al. 2005). Mass spectrometry analysis of human eIF3 has revealed three stable modules, one of which resembles that of yeast eIF3 core complex (Zhou et al. 2008). UV crosslinking and hydroxyl radical probing of mammalian eIF3 with 40S-bound mRNA has 
shown specific interactions between eIF3a and position ${ }^{-14}$ and between eIF3d and position - 8 - $^{-}$ 17 of the mRNA. This suggests that eIF3d contributes to the formation of the mRNA binding channel and that eIF3a likely forms an extension of it, which together might contribute to the processivity of the scanning (Pisarev et al. 2008). If eIF3 can hold to the solvent exposed side of $40 \mathrm{~S}$ subunit for a few elongation cycles - maybe in association with mRNA and eIF4G- then it is an ideal candidate to promote reinitiation after the translation of short regulatory uORFs (Hinnebusch 2006).

\subsection{The sub-stoichiometric $j$ subunit}

The whole eIF3 complex is capable of stably binding to the $40 \mathrm{~S}$ ribosome in the absence of other initiation factors. However, removal of its loosely associated subunit eIF3j/Hcr1 diminishes or weakens this binding. Interestingly, addition of certain oligonucleotides such as oligo(rU) or oligo(dT) can stabilize eIF3-40S interaction even in the absence of 3j (Kolupaeva et al. 2005). The N-terminal RRM domain of eIF3b is implicated in eIF3j binding and hence 40S association of eIF3. eIF3j/Hcr1 is a protein with dual functions in translation initiation as well as ribosomal RNA processing. Recent structural insight into the interaction of eIF3b-RRM and eIF3j in human has revealed that the N-terminal acidic motif of eIF3j binds to a positively charged surface of the RRM domain. This interaction is assisted by the insertion of a highly conserved tryptophan of eIF3j into a hydrophobic pocket on the surface of RRM domain. Interestingly, the exposed surface of the $\beta$-sheet of RRM, which is the RNA docking site in canonical RRMs, possesses a negatively charged surface, making its RNA binding activity unlikely (Elantak et al. 2007; Elantak et al. 2010).

Hydroxyl radical probing and modeling has placed the C-terminus of mammalian eIF3j directly in the mRNA entry channel and the ribosomal A-site. Even after TC recruitment, the C-terminus of eIF3j remains in the mRNA binding cleft. This proposes its role during scanning and/or AUG recognition by antagonizing binding of eIF1A and mRNA to the ribosome. The reduced affinity for the mRNA in presence of eIF3j is retrieved upon binding of eIF1, 1A and TC to the 40S subunit, indicating the regulatory role of eIF3j in the mRNA recruitment (Fraser et al. 2007).

\subsubsection{The role of eIF3 in ribosome dissociation}

In addition to translation initiation, eIF3 functions in disassembly of $80 \mathrm{~S}$ ribosome. After peptide release induced by eRF1/eRF3/GTP complex, eIF3 promotes dissociation of post-translational complexes (post-TCs) into the $60 \mathrm{~S}$ subunit and the tRNA/mRNA-bound $40 \mathrm{~S}$ subunit. This 
function is enhanced by eIF1, eIF1A and its loosely associated subunit eIF3j. eIF1 and eIF3j subsequently induce the release of the P-site tRNA and dissociation of mRNA, respectively (Pisarev, Hellen, and Pestova 2007). In yeast eIF3j/Hcr1 has been linked to Rli1 (RNAse L Inhibitor 1), an iron-sulfur-cluster containing member of the ABC (ATP-binding cassette) superfamily of ATPases (Kispal et al. 2005; Chen et al. 2006). The fact that Rli1 interacts with components of translation initiation (eIF3j) and termination (eRF3) might indicate a functional or regulatory link between translation initiation, termination and ribosomal recycling. Later in this chapter the function of Rli1 in translation termination in yeast is discussed.

\subsection{Modularity of eIF3}

Mass spectrometry of the natively purified human eIF3 at different salt concentrations has resulted in the dissociation of several sub-complexes as a function of the ionic strength, revealing the modularity of the complex. By comparing the subunit composition of these subcomplexes, Robinson and colleagues have suggested an interaction map of eIF3 that is composed of three stable modules: eIF3 (a:b:i:g), eIF3 (c:d:e:l:k) and eIF3 (f:h:m). These modules are linked by simultaneous interactions of eIF3c with eIF3b and eIF3h (Zhou et al. 2008). In the module eIF3 (a:b:i:g), sub-modules of eIF3 (b:i:g) and eIF3 (g:i) were present, suggesting them to form a stable sub-complex together. Interestingly, one of the observed subcomplexes, eIF3 (a:b:c:i:g), is equal to the core of yeast eIF3 and has also been generated in baculovirus (Masutani et al. 2007). This indicates that the module eIF3 (a:b:i:g) forms the core of mammalian eIF3 and interacts with the other two modules via eIF3c. The other seven "functionally dispensable" subunits ( $\mathrm{d}, \mathrm{e}, \mathrm{f}, \mathrm{h}, \mathrm{k}, \mathrm{l}$ and $\mathrm{m}$ ) are located in the periphery of the core module.

Characterization of the post-translational modifications of human eIF3 has defined 29 phosphorylation sites and several other modifications within the eIF3 complex. Most of the phosphorylations occur on eIF3a, eIF3b, eIF3c, eIF3f, eIF3j and, to a much lesser extent, eIF3g. Four subunits (h, i, k and $\mathrm{m}$ ) were found to dissociate preferentially and therefore likely to be on the periphery of the complex. Interestingly, none of these four subunits were found to be phosphorylated, pointing to an intriguing link between phosphorylation and location within the complex (Damoc et al. 2007).

\subsubsection{6. eIF3, proteasome and COP9 signalosome}

Together with two other multi-subunit complexes, COP9 signalosome (CNS) and 26S 
proteasome, eIF3 controls the life span of proteins. In multicellular eukaryotes, the "lid" subcomplex of $26 \mathrm{~S}$ proteasome, CNS and eIF3 share a common architecture. It is comprised of six subunits harboring the hallmark PCI (Proteasome, $\underline{\mathrm{CNS}}$, Initiation factor) domain and two subunits with MPN (MprI/ $\underline{\operatorname{PadI}} \underline{\mathrm{N}}$-termini) domain (Scheel and Hofmann 2005). The PCI domain is defined by several TPR-like helical repeats followed by a globular winged helix subdomain (Figure 1.8 A and B). It is believed to serve a scaffolding function, which regulates the proper complex assembly (Scheel and Hofmann 2005; Dessau et al. 2008). The MPN domain is more conserved and might have originated from a metal-binding motif (Figure 1.8 C; Pick, Hofmann, and Glickman 2009; Maytal-kivity et al. 2002).

Despite similarities in the subunit architecture, each complex performs a distinct function in the cell. eIF3 enhances the translation initiation by binding to other initiation factors, mRNA and the small ribosomal subunit. CNS regulates diverse cellular processes including signal transduction, gene expression and cell proliferation (Schwechheimer and Deng 2001). The best understood function of CNS is to promote recycling of cullin-RING ubiquitin ligases by removal of the ubiquitin-like Nedd8/Rub1 modifier from the cullin subunit (Wei, Serino, and Deng 2008). The proteasome lid carries out proteolysis of polyubiquitin-conjugated substrates (Sharon et al. 2006). The proteasome lid and the CNS show a high degree of analogy, with a clear 1:1 correspondence between the paralogous sets of MPN and PCI subunits (Kim et al. 2001). There is also a close functional resemblance between them: both Rpn11 of the proteasome lid and Cns 5 of the CNS have isopeptidase activity in their zinc-coordinated MPN motifs. eIF3, however, diverges from the other two complexes by having additional non-PCI/MPN subunits (eIF3b, d, $\mathrm{g}, \mathrm{i}, \mathrm{j}$ ). Its two MPN subunits (eIF3f and eIF3h) lack the residues necessary for metal binding and therefore are believed to be catalytically inactive. These two subunits are absent in several unicellular eukaryotes such as budding yeast (Maytal-kivity et al. 2002).

Yeast eIF3 is composed of two PCI domain-containing subunits (eIF3a and eIF3c) and four nonPCI/MPN subunits (eIF3b, g, i, j). Affinity purification and highly sensitive LC-MS/MS analysis of fission yeast eIF3 has revealed its interactome containing 230 proteins which are assembled into a large supercomplex; the translosome. The translosome is composed of different proteins involved in protein synthesis as well as degradation, such as translation initiation and elongation factors, tRNA synthetases, $40 \mathrm{~S}$ and $60 \mathrm{~S}$ subunits, chaperones, proteasome and ribosome biogenesis factors (Sha et al. 2009). 
A

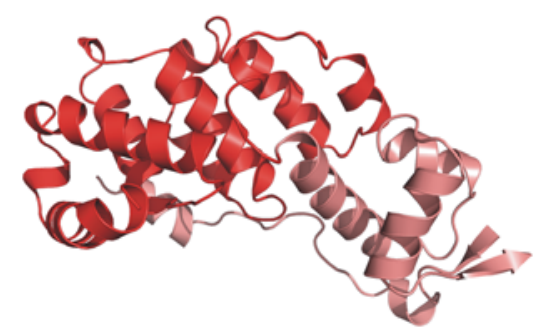

B

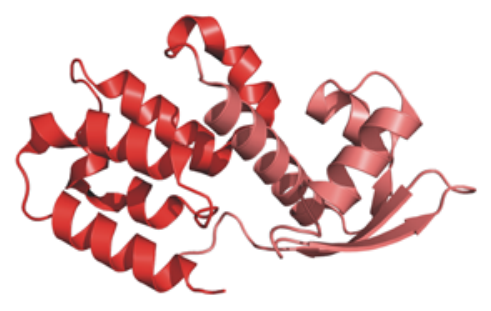

C

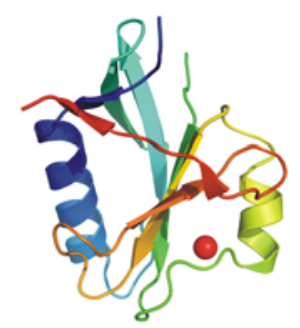

Figure.1. 8. Structure of some of the $\mathrm{PCl}$ and MPN domains within $\mathrm{PCl}$ complexes. (A) Crystal structure of human elF3K (PDB code 1RZ4) shows an example of a PCI domain. TPR-like repeats and the winged helix motif are depicted in dark red and salmon red, respectively. (B) Csn7, a subunit of COP9 signalosome, is another example of a PCl domain (PDB code 3CHM). The color codes is similar to panel A. (C) Crystal structure of MPN domain as found in Rpn11 and Csn5, the catalytic subunits of proteasome lid and COP9 signalosome, respectively, shows similarity to the metaloproteases (PDB code 1R5X). The catalytic zinc atom is depicted as a red sphere.

\subsubsection{Translational control at initiation level}

Initiation step of translation is a key point for regulation of protein synthesis as it prevents synthesis of proteins which are not immediately needed or are wrongly translated. There are two general strategies to achieve this: by mechanisms which affect initiation factors and ribosome, or mechanisms which specifically target some mRNAs either through sequence-specific RNAbinding proteins or via microRNAs (miRNAs). The first mechanism impacts on almost all scanning-dependent initiation events whereas the second one is selective for some mRNAs (for a comprehensive review see Jackson, Hellen, and Pestova 2010). The well-understood examples of initiation factor-dependent regulation are control of availability of eIF2 and eIF4 by phosphorylation.

\subsubsection{1. eIF2 phosphorylation and translation of uORFs}

As mentioned before, eIF2 has to be recharged with GTP via the function of eIF2B in order to participate in the next round of initiation. Phosphorylation of eIF2 $\alpha$ on Ser51 converts eIF2-GDP into an inhibitor, rather than a substrate, of eIF2B, leading to a decrease in the amount of available TC. The decrease in TC levels in the cell generally has a negative impact on overall translation, but induces translation of certain mRNAs, which contain several short upstream open reading frames (uORFs). The best example in this regard is the activation of the translation of yeast transcriptional activator GCN4 which has four short uORFs. After translation of the 5'most uORF (uORF1), post-termination 40S subunit resumes scanning and will reinitiate at uORFs2, 3 and 4 if there are enough eIF2-TCs. The scanning and reinitiation, however, do not 
take place after initiation at the last uORF which leads to translational silencing of the GCN4 gene. eIF $2 \alpha$ phosphorylation by amino acid starvation-activated Gen 2 reduces the levels of TC, leading to bypassing of uORFs2-4 and resuming initiation at GCN4 gene. Gcn4 is a transcription factor which induces biosynthesis of amino acids. In mammalian cells four different stressactivated kinases phosphorylate eIF2 $\alpha$ on Ser51: haem-regulated kinase (EIF2AK1) in erythroid cells, PKR (EIF2AK2) in response to double-stranded RNA in viral infections, PERK (PRL-like endoplasmic reticulum kinase or EIF2K3) in response to misfolded proteins in ER lumen and GCN2 (EIF2K4), a homolog of yeast GCN2, in amino acid starvation. Phosphorylation of eIF2 $\alpha$ at Ser51 by any of these kinases results in translation of ATF4 mRNA by a mechanism which is fundamentally similar to that of GCN4 in yeast, leading to transcriptional activation of stress response genes.

\subsubsection{Translational control by affecting cap recognition}

Cap binding by the eIF4F complex can be inhibited by members of eIF4E homolog proteins family, 4E-HPs (Raught and Gingras, 2007). These proteins compete with eIF4G for a shared binding site on eIF4E (Marcotrigiano et al. 1999). Hypophosphorylated 4E-BPs bind strongly to eIF4E, whereas its phosphorylation, mainly by mTOR, weakens this interaction (Raught and Gingras, 2007). eIF4E can also get phosphorylated on Ser209 by MNK1 and MNK2, which bind eIF4G-CTD, affecting translational efficiency.

\subsubsection{Translational control by mRNA binding proteins}

Sequences at both 5' and 3' untranslated regions are prone to regulation by sequence specific proteins. Two examples of translation regulation by 5' UTR-binding proteins are the poly-A binding protein (PABP) mRNA and many ribosomal proteins and translation elongators' mRNAs. There is a stretch of poly-A close to the 5' cap of PABP mRNA to which PABP binds and down regulates its own translation when there is too much of it present (De Melo Neto et al. 1995). PABP also binds the 3' poly-A tail of mRNAs and upon interaction with eIF4G promotes circularization of mRNA which is believed to confer a translational advantage to polyadenylated mRNAs over non-polyadenylated mRNAs when the amount of initiation factors or ribosome is limited (Proweller and Butler 1997; Borman, Michel, and Kean 2000). Another strategy for 3'specific regulation of translation initiation is sequence-specific binding of protein $\mathrm{X}$ to the 3 , UTR which via an adaptor protein $\mathrm{Y}$ interacts with a cap-recognizing protein $\mathrm{Z}$, resulting in formation of an inhibitory closed loop. Protein $\mathrm{Z}$ can be either canonical eIF4E or its paralogs. This mechanism plays an important role during development of many multi-cellular organisms; 
e.g. on progesterone-induced maturation of Xenopus laevis many of these factors get phosphorylated and subsequently degraded, leading to the activation of translation of their mRNAs (Minshall et al. 2007).

\subsubsection{Translational control via microRNAs}

MicroRNAs (miRNAs) are short ( 22 nt) oligonucleotides, which bind to mRNA in a sequencespecific manner and either inhibit translation or destabilize the mRNA or both. It is estimated that approximately half of the human genome is controlled by miRNAs. After being processed from their precursors, miRNAs are loaded on RNA-induced silencing complex (RISC) which targets and inhibits protein translation from specific mRNAs (Hammond et al. 2001; Hutvágner et al. 2001). Argonaute protein (AGO) is associated with paired miRNA-mRNA and binds to other peripheral proteins like RCK helicase and GW182. It was recently shown that the GW182 silencing domain binds PABP, which in turn recruits the complex of deadenylating enzymes (Fabian et al. 2009; Zekri et al. 2009). This interaction also competes with eIF4G-PABP which facilitates translation by formation of the closed loop. Therefore, disruption of the closed loop may also play a role in translational repression.

\subsection{Translation elongation}

During elongation, a new amino-acyl tRNA is recruited to the ribosomal A-site in a complex with eukaryotic elongation factor1A (eEF1A). eEF1A is the homologous to the prokaryotic EFTu and binds aminoacyl tRNA in a GTP-dependent manner to form a ternary complex (TC). The correct codon-anticodon base-pairing triggers the hydrolysis of GTP by eEF1A. GDP-eEF1A has low affinity for the aminoacyl tRNA and leaves the ribosome. In order to participate in the next round of elongation, eEF1A has to be recharged with GTP, in a manner which requires eEF1B, the functional homolog of bacterial EF-Ts.

As soon as aminoacyl-tRNA is accommodated in the A-site, it forms a peptide bond with the Psite peptidyl-tRNA. This is mediated by the peptidyl transferase center (PTC) on the 60S subunit, which is built up of highly conserved rRNA elements. Following peptide bond formation, the subunits undergo a ratchet-like relative rotation, in which the 3 ' ends of the tRNAs in the $\mathrm{A}$ and $\mathrm{P}$ sites move to the $\mathrm{P}$ and $\mathrm{E}$ sites, respectively, assuming a hybrid state. Translocation is accelerated by eEF2, the eukaryotic homolog of bacterial EF-G in a GTP dependent manner. After each translocation, the peptidyl-tRNA and a new codon are accommodated in the P and A sites, respectively (reviewed in Rodnina and Wintermeyer 2009). 
Elongation will continue until a stop codon enters the A-site. Since elongation was out of the scope the work of this thesis, it is not mentioned in further details.

\subsection{Translation termination and ribosomal recycling}

Translation termination and ribosomal recycling are well characterized in prokaryotes. The Asite stop codon deciphering and subsequent peptide release are mediated by the prokaryotic class I release factors RF1 or RF2. According to the direct recognition model based on a tripeptide anticodon, a conserved linear Pro-Ala-Thr (PAT) motif in RF1 decodes UAA and UAG, whereas RF2 uses a Ser-Pro-Phe (SPF) motif to decipher UAA and UGA (Ito et al, 2000, Nakamura and Ito, 2002). In case of RF1, it has recently been shown that the stop codon is recognized in a pocket formed by conserved PAT motif of RF1 and elements of 16S ribosomal RNA. The codon and the 30 subunit A-site undergo an induced fit which results in stabilization of a conformation of RF1 that promotes its interaction with the peptidyl transferase center (Laurberg et al, 2008). The class II procaryotic release factor RF3 uses GTP hydrolysis to recycle class I factors upon peptide release (Zavialov et al, 2001). RF3 binds GDP three orders of magnitude stronger than GTP which implies that the free RF3 exists in the GDP-bound form. Therefore, RF3 must enter the ribosome in the GDP-bound state, and the ribosome with a class I RF bound to the ribosomal A-site programmed with a proper stop codon serves as its guanine nucleotide exchange factor (Zavialov et al, 2001). After the hydrolysis of the peptidyl-tRNA ester bond, GTP binds to RF3 and induces conformational changes, which in turn pushes the class-I RF out of the ribosome. Subsequent hydrolysis of GTP yields GDP-bound RF3 that has low affinity for the ribosome and dissociates easily. RF3 dissociation leaves out the post-TC, which is composed of 70S ribosome, mRNA and the P-site deacylated tRNA (for review see Kisselev et al, 2003 and the references therein).

Eukaryotes have only one class-I RF (eRF1), which recognizes all three stop codons. eRF1 consists of three distinct domains (Song et al, 2000). Domain I recognizes stop codon in the small subunit A-site and harbors the highly conserved TASNIKS and YxCxxxF motifs (Bertram et al, 2000; Frolova et al, 2002; Sei-Nebi et al, 2002). Domain II possesses the highly conserved GGQ motif that interacts with the peptidyl transferase center (PTC) and triggers peptidyl-tRNA hydrolysis (Frolova et al, 1999; Seit-Nebi et al, 2001). Finally, domain III interacts with eRF3 (Ito et al, 1998; Kisselev and Buckinbham, 2000). eRF3 is an essential protein in eukaryotes with a GTP binding domain which shares homology to that of prokaryotic RF3. It has ribosomedependent GTPase activity that is stimulated by eRF1 in a codon-independent manner (Frolova 
et al, 1996). A short sequence (GILRY) at the C-terminal domain of eRF1 is crucial for its binding to eRF3 and subsequent induction of its GTPase activity (Merkulova et al, 1999; Frolova et al, 2000). This short sequence is suggested to act as an arginine finger which is common to all GTPase activating proteins (Kisselev et al, 2003).

Recently, eIF3, eIF1 and eIF1A were shown to be involved in eukaryotic ribosomal recycling at low $\mathrm{Mg}^{2+}$ concentration (Pissarev et al, 2007). In their model of ribosome recycling, Pestova and colleagues have suggested that eIF3 is the principal factor, which promotes dissociation of $40 \mathrm{~S}$ and $60 \mathrm{~S}$ subunits. eIF1 and eIF1A help eIF3 by binding to the intersubunit surface of $40 \mathrm{~S}$ subunit. eIF3 exerts its function either by a direct penetration of one of its lobes to the intersubunit surface (Siridechadilok et al, 2005) or by mediating changes in the subunit interaction that allow eIF1 and eIF1A to access their binding interface. Dissociation of deacylated tRNA and mRNA is later mediated by eIF1 and eIF3j, respectively (Pissarev et al, 2007).

The essential ATP-binding cassette (ABC) protein RLI is one of the most highly conserved proteins in evolution. Most of the proteins belonging to the $\mathrm{ABC}$ superfamily are membrane transporters (McKeegan et al., 2003) that mediate ATP-driven unidirectional transport of a variety of molecules across cell and organelle membranes. Unlike these transporters, the yeast protein Rli1p is a soluble protein (Dong et al., 2004; Kispal et al., 2005; Yarunin et al., 2005) and in this organism, it is mainly localized in the cytosol (Kispal et al., 2005; Yarunin et al., 2005). Also, in contrast to other members of the ABC superfamily, Rlilp contains two additional $\mathrm{N}$-terminal $[4 \mathrm{Fe}-4 \mathrm{~S}]^{2+}$ clusters (Barthelme et al., 2007), which are essential for protein function. The protein was originally identified in human as an inhibitor of RNase L, which cleaves double-stranded RNA in the interferon response of human cells to viral infection (Bisbal et al., 1995). Hence, RNase L Inhibitor (RLI) was thought to represent a new component of the interferon-regulated 2'-5' oligo-A pathway. Moreover, Rli1p was shown to perform a crucial function in the post-translational assembly of HIV-1 capsids in vitro by direct binding to several virus proteins including gag (Zimmerman et al., 2002). All these proposed functions, however, do not explain why Rli1p is essential since RNase L neither exists in lower eukaryotes such as yeast nor in archaebacteria.

Notably, the C-terminal domain of yeast Rlilp shares $25 \%$ to $30 \%$ identity on amino acid level to four other soluble yeast proteins of the ATP-binding cassette family that interact with ribosomes and function in translation elongation (YEF3, HEF3; (Triana-Alonso et al., 1995)) 
translational control (GCN20 (Vazquez de Aldana et al., 1995), (Marton et al., 1997)) or stimulation of 40S and 60S ribosome biogenesis (ARB1; (Dong et al., 2005)). Recent evidence indicates that Rlilp complements this list of soluble ABC proteins in yeast with a proposed function in protein synthesis and ribosome biogenesis (Dong et al., 2004; Kispal et al., 2005; Yarunin et al., 2005). Depletion of Rlilp was shown to result in strong nuclear export defect of both the small and the large ribosomal subunit (Kispal et al., 2005; Yarunin et al., 2005). Depletion of Rlilp also diminished the protein synthesis activity of cytosolic ribosomes (Kispal et al., 2005), and evidence was provided that Rlilp is required for efficient formation and stabilization of 43S and 48S pre-initiation complexes (Dong et al., 2004).

A role in translation was further proposed for the vertebrate as well as Drosophila and Caenorhabditis RLI orthologs (Andersen and Leevers, 2007; Chen et al., 2006; Coelho et al., 2005). In yeast, Rlilp was specifically found to be associated with components of the eukaryotic translation initiation machinery: eIF2, eIF5 and, in particular, the translation initiation complex eIF3. One component of the eIF3 complex, the protein Hcr1/eIF3j, was visibly enriched in Rli1p-TAP purifications (Yarunin et al. 2005). This, together with preliminary yeast-two-hybrid experiments (Kispal et al. 2005) suggest that Hcr1p and Rli1p may directly bind to each other and that Hcrlp may link Rlilp to the eIF3 complex and translation initiation.

A novel interaction between Rli1 and eRF3 and, to a lesser extent to eRF1, was recently discovered. This finding suggested the function of Rli1 in translation termination in yeast and proposed Rli1 as a candidate source of energy in ribosomal recycling. The fact that Rli1 interacts with components of translation initiation (Hcr1/eIF3j) and termination (eRF3) might indicate a functional or regulatory link between translation initiation, termination and ribosomal recycling (Khoshnevis et al. 2010). In a parallel work, Pestova and colleagues showed that ribosome recycling in a wide range of $\mathrm{Mg}^{2+}$ concentration requires $\mathrm{ABCE} 1$, the mammalian homologue of Rli1. ABCE1 was shown to dissociate post-TCs into free 60S subunits and mRNA- and tRNAbound 40S subunits. To exert its function, ABCE1 hydrolyzes ATP, GTP, UTP and CTP, thus providing the source of energy for dissociation of the subunits (Pisarev et al. 2010). In the cytoplasm of yeast, eukaryotic elongation factor 3 (eEF3), a protein found in yeast and other fungi but missing in higher eukaryotes, disassembles post-translational ribosomes into their subunits, mRNA and tRNA in an ATP dependant manner (Kurata et al.2010). Interestingly, eEF3 also belongs to the ABC superfamily of ATPases and has been shown to bind to the ribosome at a position covering both subunits near the E site (Andersen et al. 2006). This position makes it suitable for its disassembling function. 


\subsection{Aims of this work}

In order to understand the mechanism of translation initiation at molecular level, structural elucidation of initiation factors is a prerequisite. One of the major challenging factors in this regard is eIF3, owing to its large size and complex network of interactions. To address the structural features of eIF3, we set out to recombinantly purify it from E.coli to get large amounts of highly pure complex. Having the recombinant complex in hand, two combinatorial approaches of single-particle cryogenic electron microscopy (cryoEM) and X-ray crystallography could be used to shed light on the structure of yeast eIF3. In vitro reconstitution of eIF3 would allow studying the kinetics and thermodynamics of the interactions between different subunits or sub-complexes. Controlled proteolytic digestion would also provide a powerful tool to determine the stable fragments or sub-complexes within eIF3, which could be later used for crystallization.

eIF3 interacts with many different proteins. One of its interesting interaction partners is Rli1, an Fe-S cluster-containing ABC ATPase. New functions for Rlil in translation termination were investigated using different biochemical means. 


\section{Chapter $2 \bullet$ In vitro Reconstitution of Yeast}

\section{elF3}

This manuscript is in preparation for submission.

In vitro reconstitution and preliminary structural and kinetic insights into the yeast elF3 complex.

Sohail Khoshnevis ${ }^{1}$, Pohl Milon ${ }^{2}$, Florian Hauer ${ }^{3}$, Holger Stark ${ }^{3}$, Marina V. Rodnina ${ }^{2}$ and Ralf Ficner $^{1 \neq}$

\footnotetext{
${ }^{1}$ Department of Molecular Structural Biology, Institute for Microbiology and Genetics, Georg-August-University Göttingen, Germany

${ }^{2}$. Department of Physical Biochemistry, Max Planck Institute for Biophysical Chemistry, Göttingen, Germany

3. 3D Electron Microscopy Group, Max Planck Institute for Biophysical Chemistry, Göttingen, Germany

† To whom correspondence should be addressed: E-mail: rficner@uni-goettingen.de
}

Authurs Contribution:

SK: designof the experiments, cloning, purifications, ribosome preparation, thermodynamic and kinetic measurments and analyses, limited proteolysis and manual particle selection for EM. PM: ribosome purification, design of the transient kinetic experiments and data analysis, $\mathrm{FH}$ : preparation of EM grids and initial image analysis, MVR: design of the transient kinetic experiments and data, HS: analysis of EM data, RF: design of the project and analysis of data. 


\begin{abstract}
Eukaryotic translation initiation is a complex event involving the orchestrated interaction of several initiation factors (eIFs). The largest of these factors, eIF3, has five stoichiometric subunits in Saccharomyces cerevisiae. These five subunits are also believed to form the core of mammalian eIF3 which is composed of thirteen subunits in total. eIF3 plays a scaffolding role for other initiation factors and promotes their binding to the 40S ribosomal subunit. Given the complexity of eIF3, all studies on translation initiation involving eIF3 so far have been performed using the natively purified complex, which does not allow to study the role of the individual subunits in details. Here, we describe in vitro reconstitution of eIF3 from Saccharomyces cerevisiae. The purified complex is active as it binds to the $40 \mathrm{~S}$ subunit in the absence of other factors. Site specific fluorophore-labeling of eIF3 also demonstrates the functionality of the reconstituted EIF3 and provides us with novel labeling strategies to study the kinetics of translation initiation. Reconstituted complex is used for cryogenic single particle electron microscopy studies and the preliminary analysis has obtained class averages which will be used for 3D reconstruction of eIF3. By applying limited proteolysis to individual subunits of eIF3 we determined stable truncated subcomplexes which could facilitate the crystallization of the complex.
\end{abstract}

\title{
2.1. Introduction
}

The initiation of translation is the most complex step in ribosome-mediated protein synthesis requiring multiple protein interactions and thus allowing an intricate regulation. In eukaryotes, this process requires the orchestrated function of up to nine different initiation factors, eIFs. eIF2 in complex with GTP recognizes the initiator methionyl tRNA to form a ternary complex (TC) and positions it in the P-site of the small ribosomal subunit. This process is enhanced by several other initiation factors. In yeast, eIFs 3, 2, 5, 1 and $1 \mathrm{~A}$ have been shown to bind to each other prior to the ribosomal binding to form a multi-factor complex (MFC) which binds to the $40 \mathrm{~S}$ ribosome to form 43S pre-initiation complex (43S-PIC) (Sonenberg and Hinnebusch 2009; Jackson, Hellen, and Pestova 2010).

eIF3 is the largest of the initiation factors with five stoichiometric subunits in budding yeast (Tif32/eIF3a, Prt1/eIF3b, Nip1/eIF3c, Tif35/eIF3g and Tif34/eIF3i) and thirteen in human. Despite its complexity, human eIF3 shares a common core with yeast eIF3 composed of eIF3a, $b, c, g$ and $i$. The sixth subunit of yeast eIF3 is Hcr1/eIF3j, a loosely associated member of the eIF3 complex which also has functions in ribosomal biogenesis (Hinnebusch 2006). eIF3 forms 
critical interactions with other initiation factors in the MFC. Tif32 and Nip1 bind to the $\beta$ subunit of eIF2 and eIF5, respectively (Valasek, Nielsen, and Hinnebusch 2002). Direct interactions between the N- and C-termini of TIF32 with RPS0A and helices 16-18 of the 18S rRNA, respectively, promote the binding of eIF3 to the $40 \mathrm{~S}$ ribosomal subunit. These interactions propose that eIF3 associates with the solvent-exposed side of the 40S subunit (Valásek et al. 2003). Cryoelectron microscopy of human eIF3 in combination with modeling revealed a fivelobed particle which together with the eIF4G occupies the solvent exposed surface of 40S ribosome (Siridechadilok et al. 2005). This model is in accordance with the yeast model (Valasek et al. 2003). Mass spectrometry analysis of human eIF3 has proposed three stable modules, one of which resembles that of the yeast eIF3 core complex (Zhou et al. 2008). UV crosslinking and hydroxyl radical probing of mammalian eIF3 with the 40S-bound mRNA has revealed specific interactions between eIF3a and position -14 and between eIF3d and position -8 to -17 of mRNA. It is therefore suggested that eIF3d contributes to the formation of mRNA binding channel and eIF3a likely forms an extension of it, which together might contribute to the processivity of the scanning of the mRNA (Pisarev et al. 2008).

The loosely associated subunit of eIF3, Hcr1/eIF3j, is shown to be critical for the ribosome binding by eIF3. Removal of Hcr1/eIF3j diminishes or weakens the interaction so that it cannot withstand the gradient centrifugation anymore. The N-terminal RNA recognition motif (RRM) domain of eIF3b interacts with eIF3j and promotes 40S subunit binding of eIF3. Moreover, mutation of the ribonucleoprotein (RNP) 1 motif within the eIF3b-RRM increases leaky scanning, proposing a role in AUG recognition. Recent structural insight into the interaction of human eIF3b-RRM with eIF3j revealed the engagement of the N-terminal acidic motif of eIF3j in binding to a positively charged surface of the RRM domain away from the exposed surface of the beta sheet. This interaction is assisted by insertion of a highly conserved tryptophan of eIF $3 \mathrm{j}$ into a hydrophobic pocket in the RRM domain. Interestingly, the RNA binding surface of human eIF3b-RRM possesses a negatively charged surface, making its RNA binding activity unlikely (Elantak et al. 2010). Analysis of eIF3b-RRM domain in yeast has suggested that although most probably it uses the same mechanism of interaction with eIF3j as in human, it is a canonical RNA binding protein. This enables it to play a role in either recruitment of eIF3 to the ribosome or $48 \mathrm{~S}$ complex formation by facilitating mRNA interaction with eIF3 (Khoshnevis et al, 2010).

Here we report on the in vitro reconstitution of yeast eIF3 assembled from the recombinantly expressed subunits. In vitro reconstitution allowed us to study the sub-complexes within eIF3 and the thermodynamics of their interactions, gaining insights into the order of the complex 
formation. The structure of the reconstituted complex was studied by single-particle cryogenic electron microscopy (cryo-EM). Analysis of different sub-complexes by EM will enable us to determine the position of different subunits within the complex. Site-specific labeling of recombinant eIF3a/Tif32 by a fluorescent probe prior to the reconstitution of the complex provided us with new and invaluable tool to study translation initiation events by transient kinetic methods. Limited proteolysis of different sub-complexes of eIF3 allowed defining stable cores inside the complex which will facilitate future structural studies of the complex by X-ray crystallography.

\subsection{Results}

\section{Protein expression and purification}

Different subunits of yeast eIF3 were cloned into either N- or C-terminal His-tag and N-terminal GST-tag containing expression vectors. The protein expression of each of the DNA constructs were tested in different E.coli expression strains and the best ones were chosen based on the highest expression of soluble protein. A list of the DNA constructs as well as their expression strains which were finally used for protein purification are provided in table 2.

Most of the eIF3 components including Tif32, Nip1, Tif34 and Tif35 could be purified individually. However, attempts to purify Prt1 with a C-terminal His-tag failed, mainly yielding a degradation product starting at amino acid 181 and preserving the His-tag. Therefore, Prt1 was purified in a complex with two other large subunits of eIF3, Tif32 and Nip1. These three proteins form a stable complex, which is stable in the presence of $500 \mathrm{mM} \mathrm{NaCl}$. The only remaining impurities of this complex are minor degradation products as well as the chaperone Hsp70. Incubation with $\mathrm{ATP} / \mathrm{MgCl}_{2}$ removes the majority of the chaperone contamination (data not shown). The most prominent degradation product is a band at about $70 \mathrm{kDa}$ which was determined to be the same degradation product of Prt1/3b that we had observed before.

\section{Formation of eIF3 and its subcomplexes}

eIF3 reconstitution was achieved by mixing two main sub-complexes of it: Tif32/Prt1/Nip1 and Tif34/Tif35. As depicted in figure 2.1, a shift to a lower elution volume of the mixture of the sub-complexes, distinct from the void volume of the column, was observed indicating the formation of the hetero-pentameric eIF3. Analysis of this peak by SDS-PAGE confirmed the presence of all five subunits. 
A

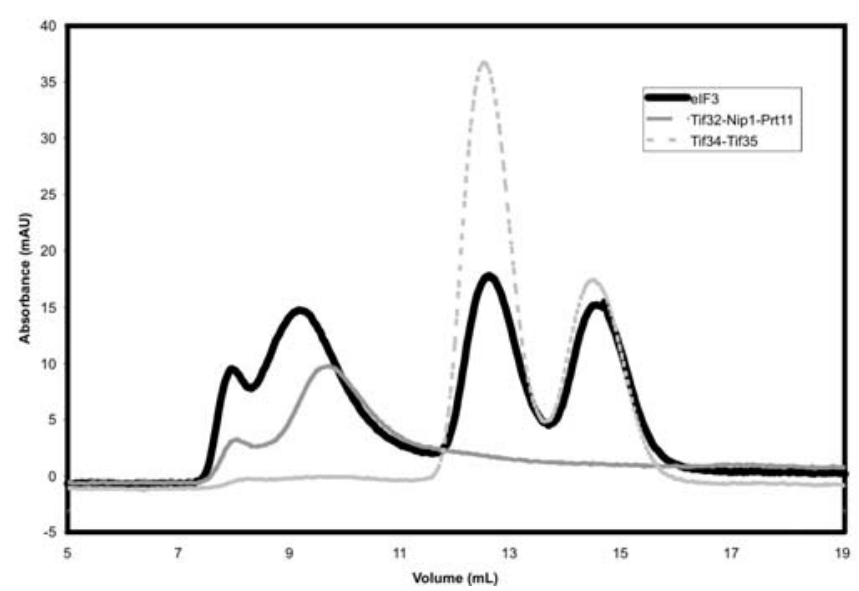

B

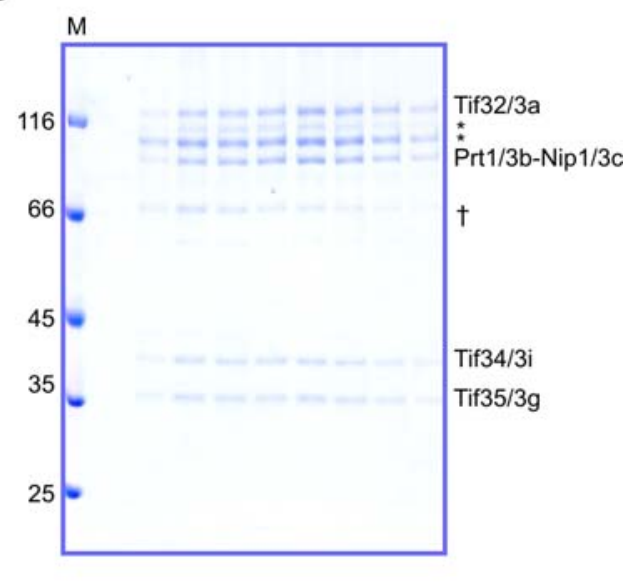

Figure.2. 1. In vitro reconstitution of yeast elF3. (A) Overlay of three size exclusion chromatograms: the dashed light grey line represents the Tif34/Tif35 complex, dark grey line belongs to the Tif32/Prt1/Nip1 complex and solid black line depicts the hetero-pentameric elF3 complex. (B) Analysis of the elF3 peak (panel A) on SDS-PAGE indicates the presence of all five subunits. Two truncations of Tif32 are marked by asterisks. + stands for a stable degradation product of Prt1 starting from amino acid 181 to the end.

In vitro reconstituted eIF3 (eIF3 ${ }^{\text {rec }}$ ) was compared to natively purified eIF3 (eIF3 ${ }^{\text {nat }}$ ) by SDSPAGE and mass-spectrometry (Figure 2.2). While Tif32 in eIF3 ${ }^{\text {rec }}$ degrades to produce two slightly shorter fragments, it remains preserved in eIF $3^{\text {nat }}$. For eIF ${ }^{\text {nat }}$ there are two bands which both correspond to eIF5 contamination. However, no difference was detected between them by mass-spectrometry. There are also two bands for Tif34 in eIF3 ${ }^{\text {nat }}$ whose differences could not be distinguished by mass-spectrometry.

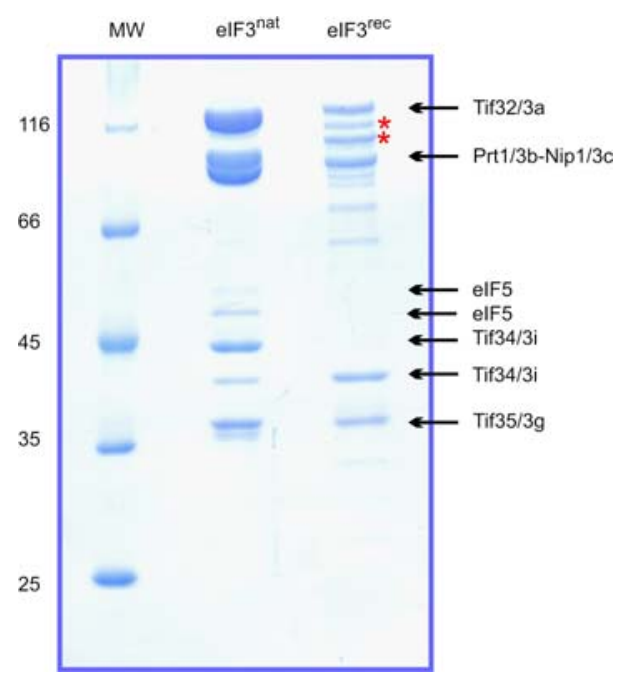

Figure.2. 2. Comparison of the recombinant and native elf3. Note that the native preparation is contaminated with elF5. There is an additional band for Tif34 which is larger than the recombinant Tif34 which might have been produced by post-translational modifications. Two degradation products of Tif32 are marked by red asterisks. MW stand for molecular weight marker, numbers for MW are in KDa. 
The stability of different sub-complexes of eIF3 was studied by analytical gel filtration. As expected, a stable binary complex was assembled by mixing Tif34 and either Tif35 or a Cterminal deletion mutant of Prt1, denoted as Prt ${ }^{181 \mathrm{C}}$. However, upon mixing Tif35/3g and Prt ${ }^{181 \mathrm{C}}$ only the elution peak broadening and no change in the retention volume was observed (Figure 2.3 A). SDS-PAGE analysis of the fractions of the elution peak revealed that in fact there are two adjacent peaks, corresponding to each of the proteins alone (Figure $2.3 \mathrm{~B}$ ). Despite the reports that Prt1 and Tif35 bind to each other, we did not observe any interaction in vitro. Hence, it is tempting to speculate that a third protein, most probably Tif34 which has been shown to interact with both of them, is bridging these two proteins in eIF3 complex.

A

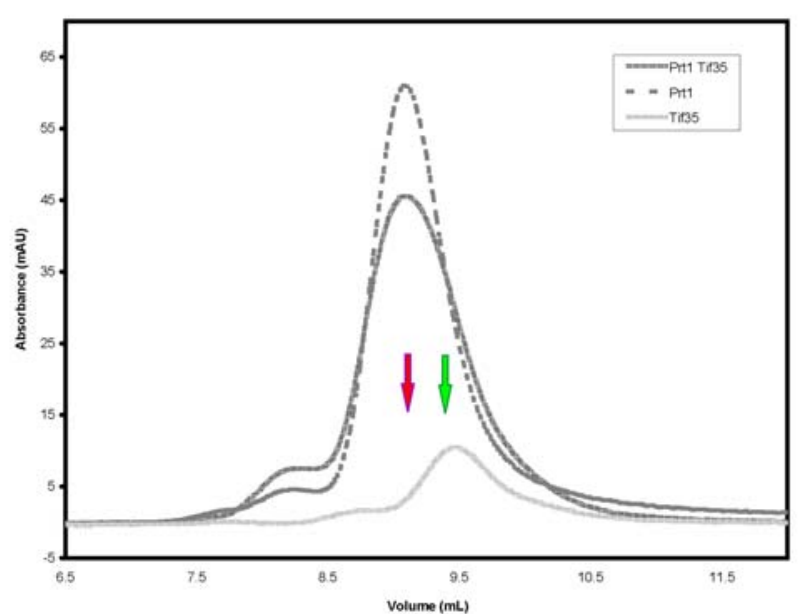

B

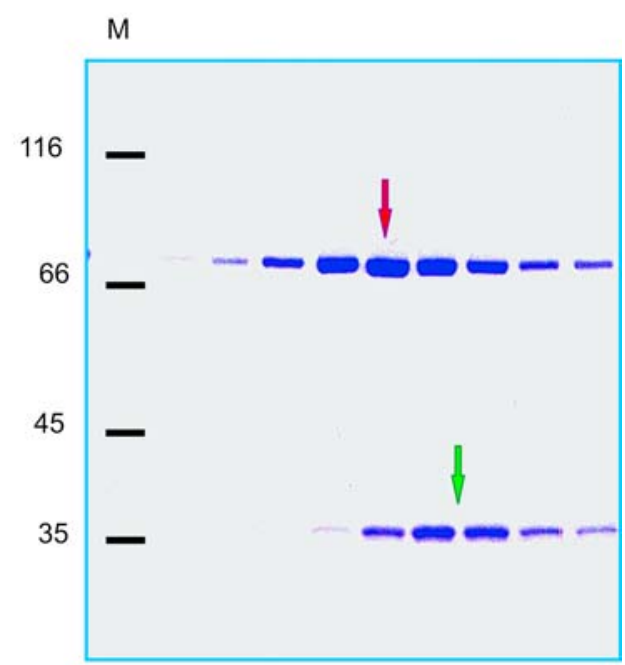

Figure.2. 3. Prt1-CTD and Tif35 do not interact in vitro. (A) Overlay of the analytical gel filtration profiles of Prt1 ${ }^{181 C}$ (dashed dark grey), Tif35 (dashed light grey) and Prt1 ${ }^{181 \mathrm{C}}-\mathrm{Tif35}$ mixture (continuous dark grey) shows a broadening of the peak in the case of the mixture without any considerable shift the retention volume compared to individual components. (B) SDS-PAGE analysis of the peak of the mixture of Prt ${ }^{181 C}$-Tif35 shows that it is in fact composed of two adjacent peaks (marked by red and green arrows) as would be judged by the broadening of the peak. Red and green arrows indicate fractions of the gel filtration peak of the mixture which are indicated by the same arrows in the panel $A$.

\section{Ribosome binding assay of eIF $3^{\text {rec }}$}

eIF3 forms a scaffold for the binding of other proteins and the ribosome. Hence, the ability to bind to the ribosome has been considered as an activity assay for eIF3. Hcr1, a substoichiometric subunit of eIF3, is known to promote the binding of eIF3 to the 40S subunit. To test whether the reconstituted eIF3 has preserved its ribosomal binding properties, the purified yeast ribosomal $40 \mathrm{~S}$ subunit was mixed with Hcrl and eIF ${ }^{\text {rec }}$ in a 4:2:1 ratio to ensure that $\mathrm{eIF}^{\text {rec }}$ is saturated with Hcr1 and the ribosome. The complex was analyzed by native-PAGE 
followed by a western blot and immunostaining against the His-tag of Tif32. A shift of the observed signal towards higher molecular weights upon addition of the 40S subunit and Hcr1 indicates the formation of a complex between eIF $3^{\text {rec }}$ and $40 \mathrm{~S}$ subunit. The same procedure was repeated for eIF $3^{\text {nat }}$. The resulting complex of eIF $3^{\text {nat }}-40 \mathrm{~S}$ had the same apparent molecular weight as eIF3 ${ }^{\text {rec }}-40 \mathrm{~S}$ complex (Figure 2.4).

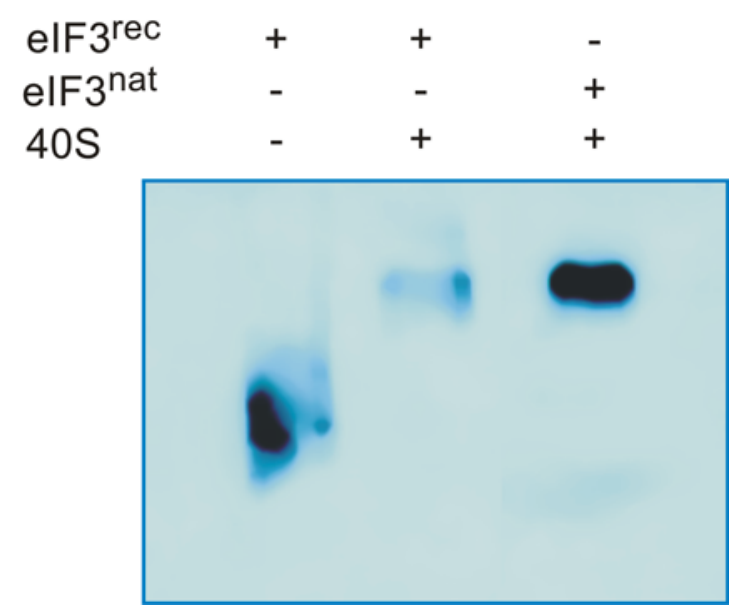

Figure.2. 4. Analysis of the binding of elF 3 to the $40 \mathrm{~S}$ ribosomal subunit on native PAGE. A shift towards high molecular weights upon mixing $40 \mathrm{~S}$ subunit and elF $^{\text {rec }}$ indicates the binding of two complexes. The resulting complex has almost the same size compared to the native elf3 bound to the $40 \mathrm{~S}$.

\section{Transient kinetic studies}

Transient kinetic methods in combination with fluorescently labeled proteins have been widely used in the field of protein synthesis (Milon et al. 2007). The most common approach for protein labeling is the usage of thiol-reactive derivatives of fluorophores to attach them to single cysteins on the surface of the proteins. Tif32 carries a single cysteine at position 190 which makes it a suitable target for the labeling with a maleimide derivative of Alexa488 (Tif32 ${ }^{*}$ ). The labeling efficiency under the working condition was about $10 \%$, which given the low concentration of the protein that was applied, yielded a low intensity signal. However, detectable signals for different reactions could be obtained, including the complex formation between Tif32* and Nip1 (Figure $2.5 \mathrm{~A}$ ). The binding scheme is biphasic: a rapid phase with a small contribution to the amplitude, and a slower phase contributing to the most of the change in amplitude, with an apparent binding constant of $1.83 \mathrm{~s}^{-1}$. Chasing of the Tif32*/Nip1/Prt1/Hcr1 complex with an excess of unlabeled Tif32 in presence or absence of the $40 \mathrm{~S}$ ribosomal subunit resulted in a decrease in the fluorescence signal only in the ribosome-bound state with a $k_{\text {off }}$ of $0.025 \mathrm{~s}^{-1}$. This indicates a probable structural rearrangement of the complex upon interaction with the ribosome (Figure $2.5 \mathrm{~B}$ ). 


\section{Initial preparations for electron microscopy}

The eIF3 ${ }^{\text {rec }}$ was used to prepare negative stain grids for electron microscopy. Initial images showed high degree of heterogeneity within the sample, showing particles with a wide range of size and shape (Figure 2.6 A).

A

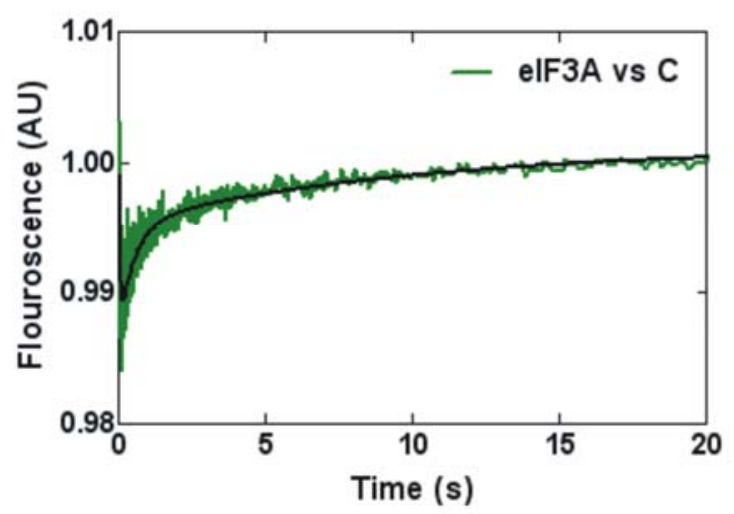

B

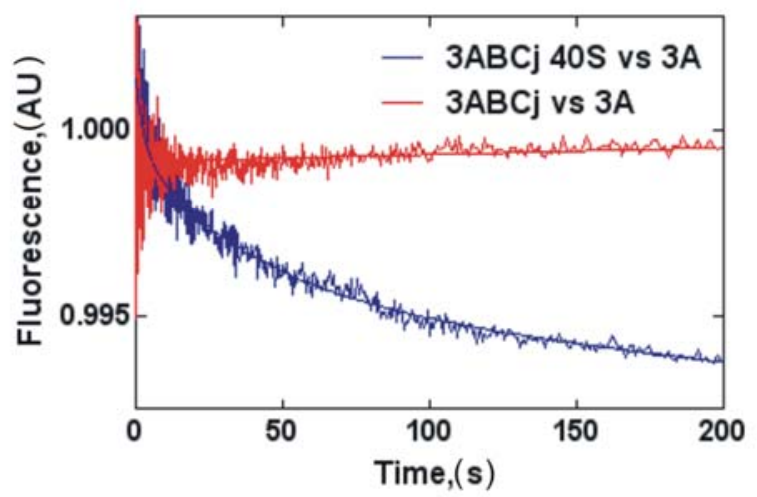

Figure.2. 5. The use of fluorescently labeled Tif32 in kinetic analysis. (A). Binding of Tif $32^{\text {Alexa488 }}$ to unlabeled Nip1. (B). Dissociation of Tif32 $2^{\text {Alexa488 }}$ from Tif32/Nip1/Prt1/Hcr1 complex in the presence of unlabeled Tif32 is enhanced in the presence of $40 \mathrm{~S}$ ribosomal subunit.

A

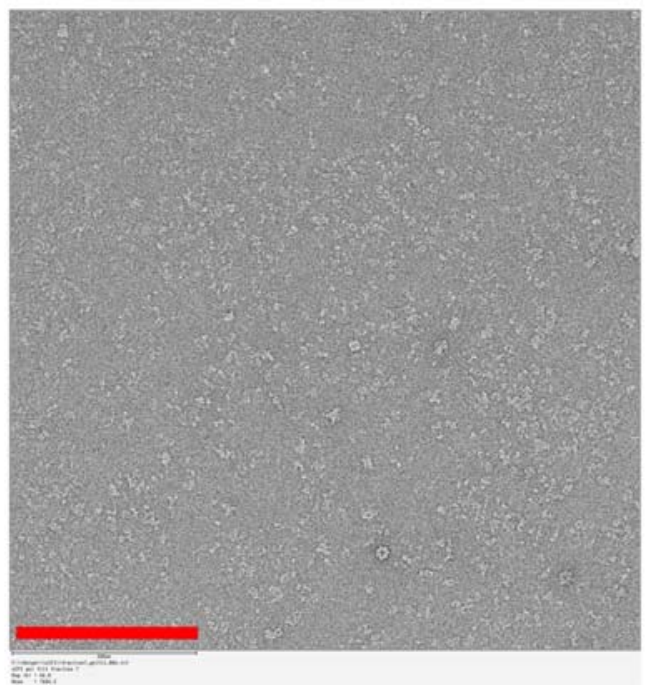

B

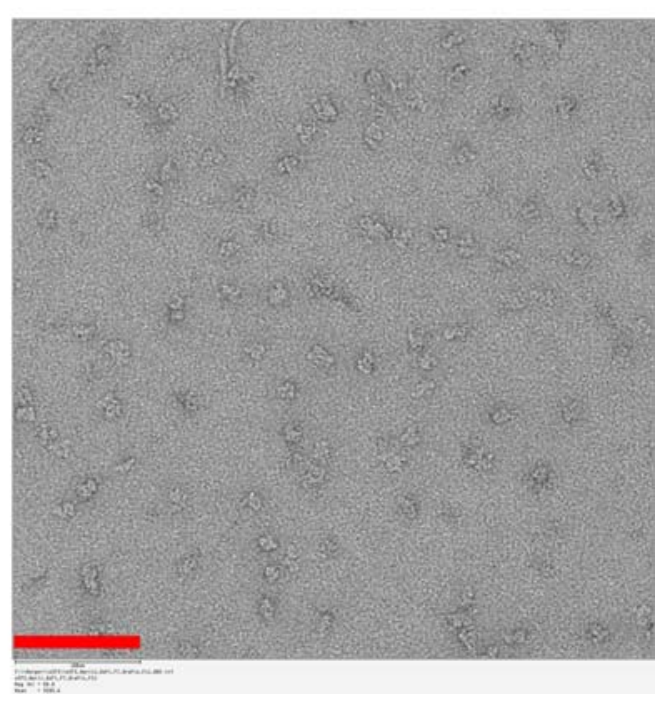

Figure.2. 6. Comparison of the EM grids before and after GraFix. (A). The purified complex shows high level of heterogeneity on the negative stain grids. Several complexes as well as GroEL contaminations are present. The red bar represents $200 \mathrm{~nm}$. (B). sample prepared by GraFix method is quite homogenous and particles are more or less of the same size. The GroEL contamination is also removed. The red bar represents $100 \mathrm{~nm}$. 
The heterogeneity of the sample on the grids might come either from the sample itself, or be due to the conditions used for grid preparation which could have resulted in dissociation of the complex. To address the source of heterogeneity in the sample and find a way to overcome it, the eIF3 $^{\text {rec }}$ complex was analyzed by size exclusion chromatography and the resulting peak containing all proteins, as verified by SDS-PAGE, was applied again on a size exclusion chromatography column. As depicted in figure 2.7, the complex has partially dissociated again. The interaction between Tif34 and Prt1 within the complex therefore seems to be not strong enough to withstand the gel filtration conditions, whereas Tif34 and Tif35 are strongly bound to each other.

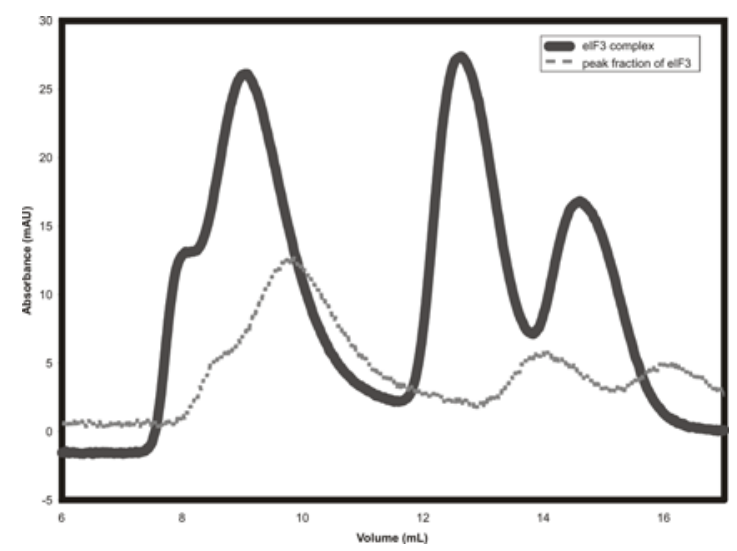

Figure.2. 7. Dissociation of elF3 into sub-complexes. The peak fraction of the gel filtration run for elF3 reconstitution was applied immediately on the gel filtration. The result suggests partial dissociation of elF3 into a subcomplex composed of Tif32/Nip1/Prt1 and Tif34 and Tif35.

Thermodynamic studies of the complex formation between Tif34 and Tif35 using isothermal titration calorimetry (ITC) reveals the formation of a strong complex in a 1:1 ratio with a $K_{d}$ of $\sim 100 \mathrm{nM}$. The formation of this complex is driven by a net enthalpy change of more than 33 $\mathrm{kcal} \cdot \mathrm{mole}^{-1}$. In comparison, the thermodynamic analysis of Tif $34 / \mathrm{Prt}^{181 \mathrm{C}}$ interaction shows the formation of a 1:1 complex with the $K_{d}$ of $\sim 400 \mathrm{nM}$ and a net enthalpy change of about 28 $\mathrm{kcal} \cdot \mathrm{mole}^{-1}$ (Figure 2.8). This comparison provides a thermodynamic explanation for the low stability of the eIF3 ${ }^{\text {rec }}$ complex on EM grids. Together with the lack of interaction between Tif35 and Prt1 (Figure 2.3), it also suggests the existence of two distinct thermodynamically stable sub-complexes of eIF3, shedding light on the order of complex formation.

To overcome the intrinsic tendency of eIF ${ }^{\text {rec }}$ to dissociate into sub-complexes, the Grafix method was applied before preparing the EM grids (Kastner et al. 2005). The fixed samples 
showed great enhancement in the images (Figure 2.3 B). In total 31,359 particles were selected for creation of class averages. The resulting classes had high contrast and could be totally distinguished from the background (Figure 2.7). These classes will be used for future 3D reconstruction of the complex.

A

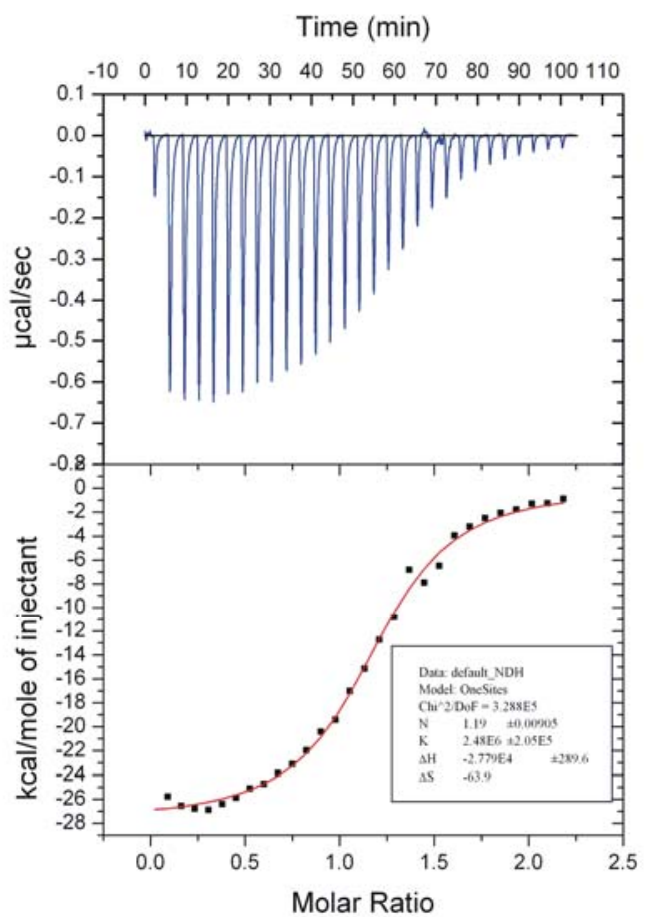

B

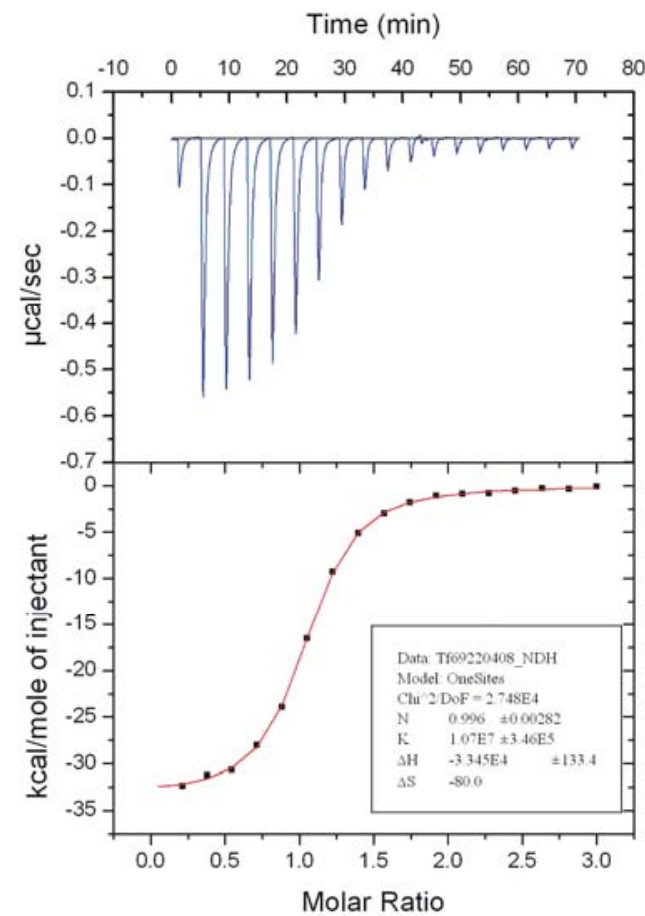

Figure.2. 8. Thermodynamic dissection of the interactions within Prt1/Tif34/Tif35 sub-complex by ITC. (A). The equimolar interaction of Prt1 ${ }^{181 C}$ and Tif34 is driven by the release of heat which compensates for the decrease in entropy. (B). Interaction of Tif34 with Tif35 is also equimolar and enthalpy driven. Comparison of the $K_{d} S$ of both interactions suggests the formation of four times tighter complex between Tif34 and Tif35. In both cases, the upper panels show raw data of heat effect (in $\mu \mathrm{cal} \cdot \mathrm{s}^{-1}$ ) of injections of Tif34 into $1.5 \mathrm{ml}$ of Prt1 ${ }^{181 \mathrm{C}}$ (A) or Tif35 (B) performed at $300 \mathrm{~s}$ intervals. The lower panels show the fitted binding isotherms. The data points were obtained by integration of heat signals plotted against the molar ratio of Tif34 to either of the interaction partners in the reaction cell. The solid lines represent calculated curves using the best fit parameters obtained by a nonlinear least squares fit.

\section{Determination of structurally stable fragments using limited proteolysis and mass spectrometry}

In order to determine the stable truncations of eIF $3^{\text {rec }}$, its different sub-complexes were subjected to limited proteolysis using various proteases and analyzed by SDS-PAGE. Stable fragments or truncated sub-complexes were analyzed via analytical gel-filtration for their structural integrity. 


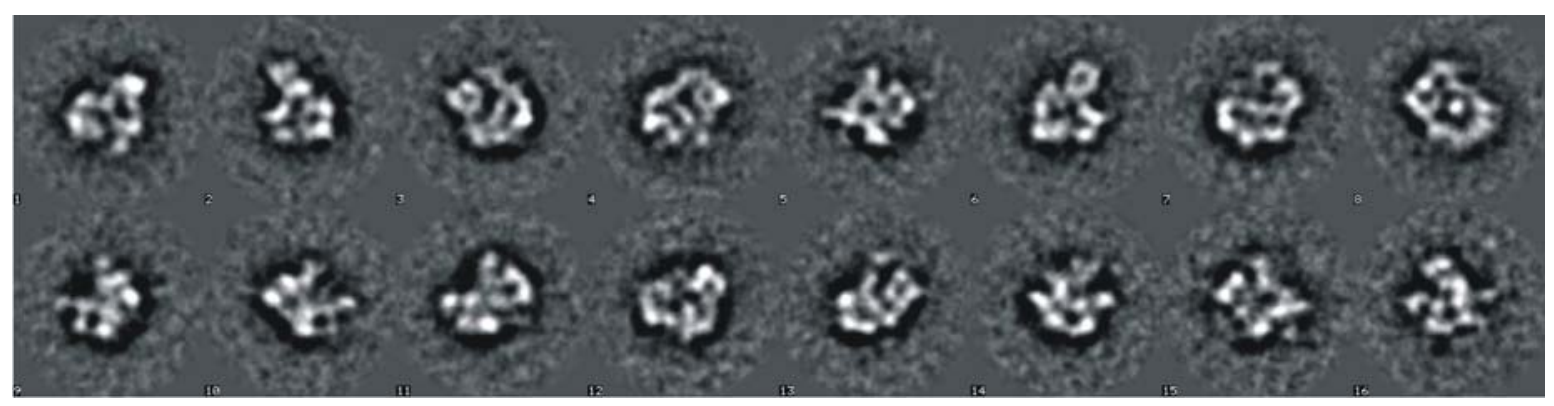

Figure.2. 9. 2D analysis of the recombinantly reconstituted elF3 complex. Multiple class averages of negatively stained samples of elF3 show a somewhat globular complex.

The digestion of the sub-complex of $\mathrm{Prt}^{181 \mathrm{C}} / \mathrm{Tif34/Tif35}$ with thermolysin generated three distinct bands after $24 \mathrm{~h}$ of incubation (Figure $2.10 \mathrm{~A}$ ), with the uppermost one corresponding to Prt $1^{181 \mathrm{C}}$ which is lacking its very C-terminal part. The middle band consisted of almost undigested Tif34 and the third one of truncated Tif35. Interestingly, the protease-treated sample when applied on gel filtration chromatography was eluted as two distinct peaks containing either

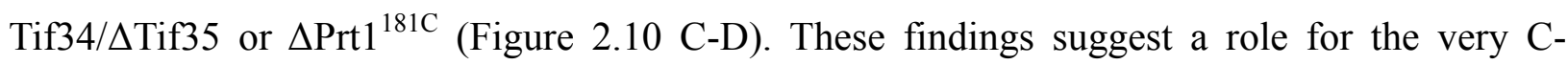
terminal part of Prt1 in the interaction with Tif34. Based on these results and the predictions of the secondary structure organization of Prt1 and Tif35, two truncated fragments of Prt1, starting at amino acid 670 ranging to the end and Tif35 spanning amino acids 14 to 150 were designed. Prt1 ${ }^{670 \mathrm{C}}$ was purified alone, whereas Tif3 $5^{14-150}$ had to be co-purified with Tif34. A stable ternary complex was formed between Prt1 $1^{670 \mathrm{C}} / \mathrm{Tif} 34 / \mathrm{Tif} 35^{14-150}$ by mixing equimolar amounts of the proteins.

Proteolytic digestion of a second subcomplex, namely Tif32/Nip1 complex with GluC generated stable fragments (Figure 2.10 B), which were eluted as a stable complex on the analytical gel filtration column (Figure 2.10 E-F). Mass spectrometric analysis of these fragments revealed that the N-terminal domain of Tif32 and the C-terminal domain of Nip1 are preserved. Using a similar rationale as described above, truncations of Tif32 and Nip1 were designed covering residues 1-494 and 240-812, respectively. The complex was purified by expressing the proteins individually and mixing the cell pellets prior to the cell lysis as described for the Tif32/Prt1/Nip1 complex.

\subsection{Discussions}

So far all the structural and biochemical studies on eukaryotic translation initiation involving eIF3 have been performed using natively purified protein. This approach has certain advantages 
such as the presence of all possible post-translational modifications which might be of importance for the proper function of the protein in vivo. However, the fact that there are substantial amounts of eIF5 co-purified with the natively purified eIF3 makes it difficult to reliably use it in biochemical and functional experiments dealing with the GTPase activity of eIF2 (Acker et al. 2007). In vitro reconstitution of eIF3 not only overcomes the problem of copurification of other initiation factors, but also shed light on the step-wise assembly of the complex. Moreover, step-wise reconstitution of the eIF3 complex also allows to compare different sub-complexes structurally, e.g. using electron microscopy, and define the position of different subunits in the complex. In addition, having all individual protein in hand and formation of different sub-complexes and their stable truncations increase the chance of looking at the structure of eIF3 by X-ray crystallography.

During the preparation of the grids for negative-stain EM, we noticed the low stability of the eIF $3^{\text {rec }}$ complex. Dissection of the thermodynamics of the individual interactions between Tif34 with either Tif35 or Prt1 revealed the formation of a stronger complex between Tif34 and Tif35, explaining why the eIF3 ${ }^{\text {rec }}$ dissociates into two stable sub-complexes. This result is in accord with the mass-spectroscopic studies on human eIF3 showing that the counterparts of Tif34 and Tif35 in human are located at the periphery of the complex, excluded from its core and tend to leave the complex more easily (Damoc et al. 2007; Zhou et al. 2008). Tif32, Nip1 and Prt1 therefore assemble the core of eIF3, forming a stable sub-complex of eIF3, which resists high concentrations of salt. This is consistent with the observation that a subcomplex of eIF3 composed of Tif32, Nip1 and Prt1 together with eIF5 is sufficient for binding to the ribosome (Valásek et al. 2003). Interestingly, despite previous reports, no interaction was observed between Prt $1^{181 \mathrm{C}}$ and Tif35 at physiological salt concentrations, suggesting that Tif34 bridges the interaction between Tif32/Nip1/Prt1 and Tif34/Tif35 sub-complexes. Using limited proteolysis a stable core within the complex of Tif32/Nip1 was determined, composed of Tif32-NTD and Nip1-CTD, which withstood proteolytic digest. The same method helped us to define the last 94 residues of Prt1 and residues 14-150 of Tif35 to be responsible for their interactions with Tif34, hence forming another stable truncated sub-complex. 
A

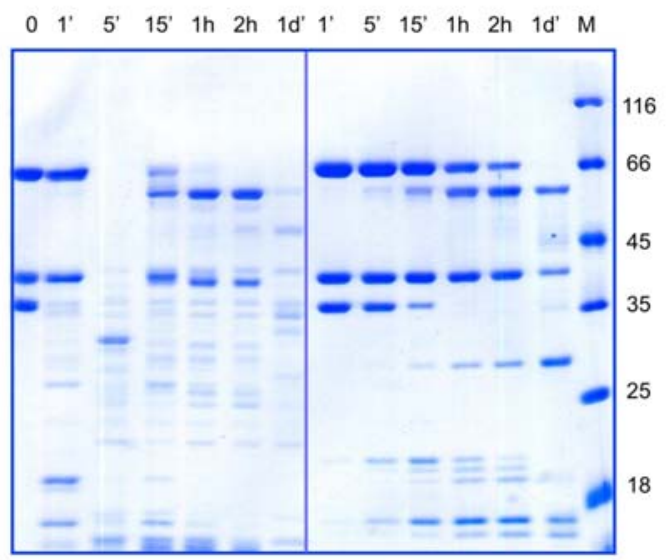

C

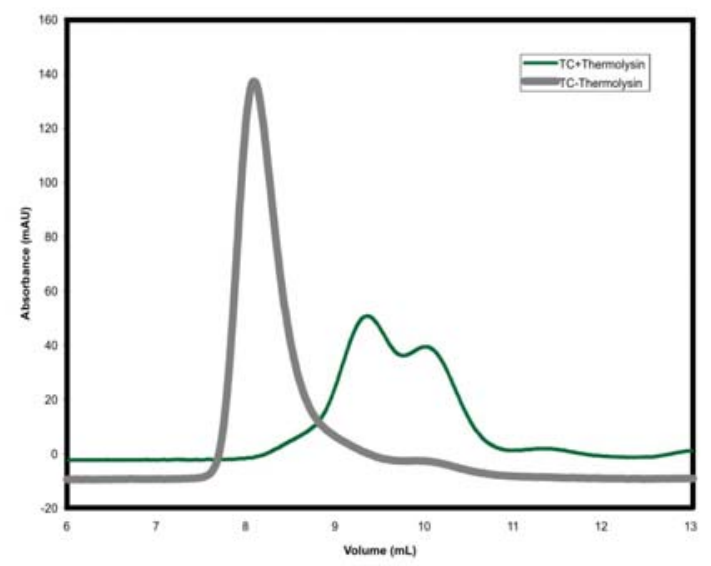

$E$

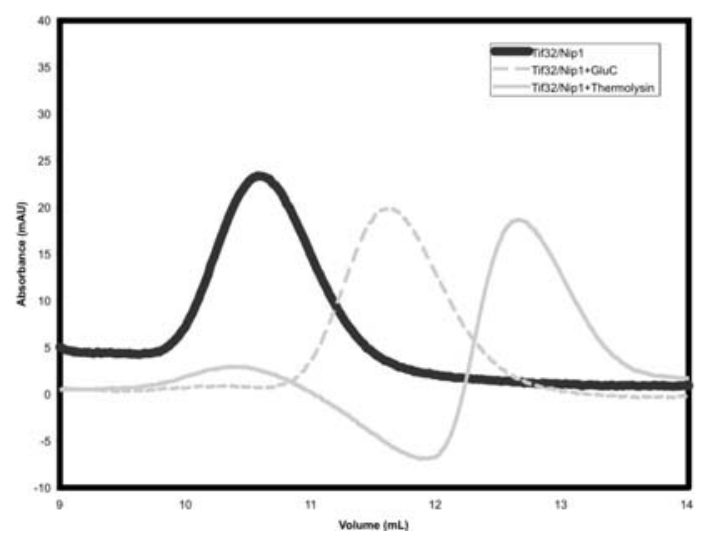

B

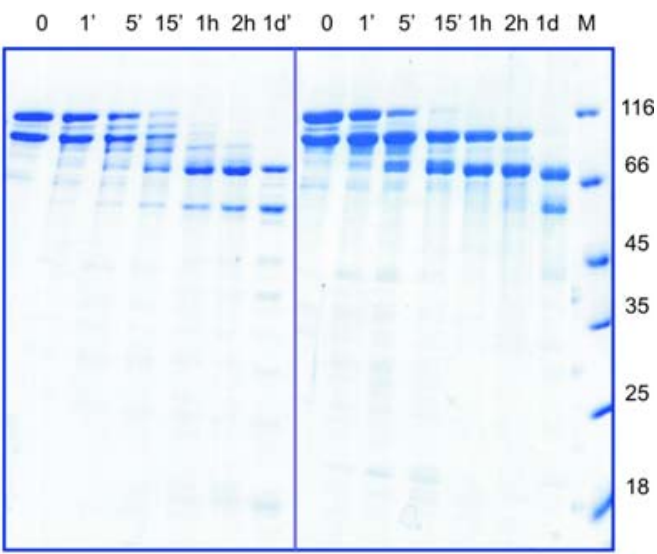

D

$\begin{array}{llllllllllll}M & 0 & 1 & 2 & 3 & 4 & 5 & 6 & 7 & 8 & 9 & 10\end{array}$

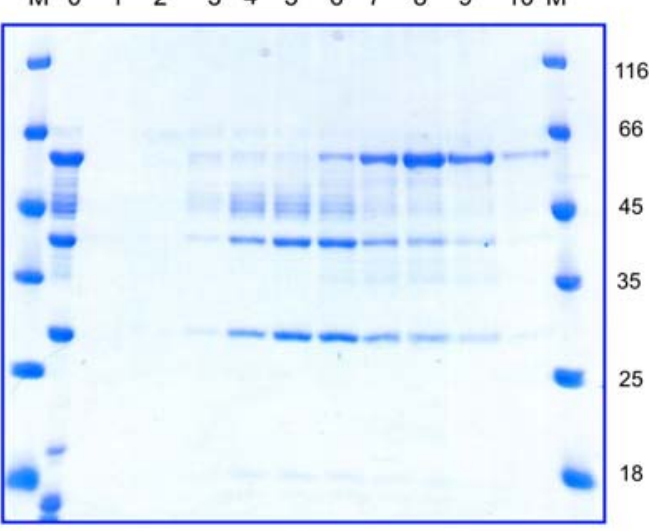

$\mathrm{F}$

$\begin{array}{lllllllllllll}0 & 1 & 2 & 3 & 4 & 5 & 0 & 1 & 2 & 3 & 4 & 5\end{array}$

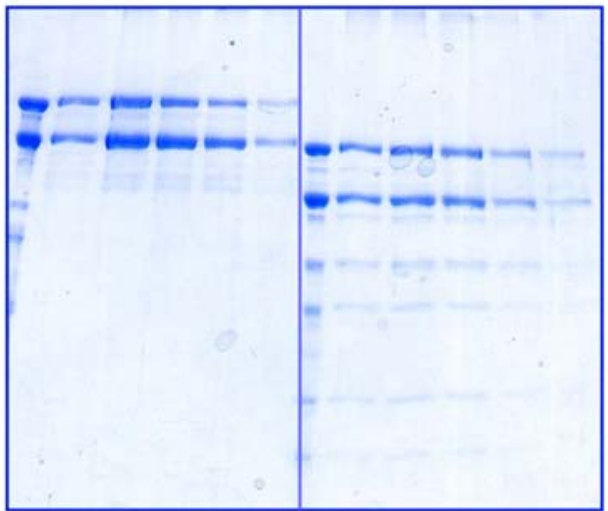

Figure.2. 10. limited proteolysis of the sub-complexes of elF3. SDS-PAGE of the proteolysis of $\mathrm{Prt1}^{181 \mathrm{C}} / \mathrm{T}$ if34/Tif35 complex (A) and Tif32/Nip1 complex (B) in the time course of one minute to one day. Results obtained by GluC and thermolysin are depicted in the left and right panels of each image, respectively. (C). Analytical gel filtration profile of the Prt1 ${ }^{181 C} /$ Tif34/Tif35 complex digested with thermolysin after one day. Resulted fragments do not form the complex as manifested by the presence of two peaks shifted to the right (dark grey) compared to the non-cleaved complex (light grey). (D). SDSPAGE analysis of gel filtration run in panel $C$ suggests the dissociation of the complex into a truncation of 
Prt1 ${ }^{181 C}$ and Tif34 in complex with a truncation of Tif35. Numbers correspond to different fractions of the peak. 0 is the sample prior to gelfiltration. (E). Analytical gel filtration of Tif32/Nip1 complex treated either with GluC for two hours (solid light grey) or with thermolysin for one day (dashed light grey). Comparison with the non-cleaved complex (solid dark grey) proposes the existence of only one complex in each case, indicating the preservation of the interactions between resulted fragments. (F). SDS-PAGE analysis of gel filtration runs in panel $E$ shows the proteolytic fragments to be bound together. Left and right panels are different fractions of the peaks after GluC and thermolysin treatments, respectively. In each case 0 is the sample prior to the gelfiltration. In all cases, $M$ stands for molecular weight marker and numbers are in $\mathrm{kDa}$.

In vitro reconstitution of eIF3 complex allowed us in this study to fluorescently label it by attaching a fluorophore to its Tif32 subunit at position $\mathrm{C}^{190}$ prior to the reconstitution. The initial results indicated $10 \%$ efficiency of labeling. Several factors can influence the labeling efficiency including the accessibility of the targeted cystein, the $\mathrm{pH}$ of the reaction buffer and the nature of the fluorophore. Moreover, the more surface-exposed the cystein is, the higher is the chance of meeting and accepting the fluorophore. The fact that there is already $10 \%$ of labeling efficiency without any optimization suggests that the Cys ${ }^{190}$ is to some extent exposed. The efficiency of cysteine labeling with maleimide fluorescent derivatives depends on the degree of protonation of its sulfur group, therefore an acidic medium is preferred. However, the optimal $\mathrm{pH}$ for high efficiency also depends of the stability of the target protein at a certain $\mathrm{pH}$. In the case of Tif32*, the protein was kept at $\mathrm{pH} 7.5$. Hence, further studies are needed to screen a range of $\mathrm{pH}$ and fluorophores for a compromise between the stability of the protein and its labeling efficiency. Our initial results indicated the power of Tif32-labeling in looking at the kinetics of interactions within eIF3 as well as eIF3 and ribosome. Binding of labeled Tif32* to unlabeled Nip1 was studied by means of stopped-flow method. The binding scheme fits to a two exponential model with an apparent binding constant of $1.83 \mathrm{~s}^{-1}$ for the main phase. The complex of Tif32 $2^{*}$ Prt1/Nip1/Hcr1 was chased with unlabeled Tif32 in absence and presence of the small ribosomal subunit. Interestingly, only in the presence of $40 \mathrm{~S}$ subunit decay in the measured signal was observed. This indicates that the dissociation rate constant of Tif32* from the Tif32*/Prt1/Nip1/Hcr1 complex is much lower than the dissociation of Tif32*, and possibly the whole complex, from the 40S. This may also indicate that upon binding of the complex to the $40 \mathrm{~S}$ subunit, a structural rearrangement occurs leading to a lower affinity of Tif $32 *$ to the ribosome and/or the complex. 


\subsection{Materials and methods}

\section{Cloning}

A general strategy was applied for the cloning of all proteins and their truncation. DNA fragments corresponding to the desired open reading frames were generated by PCR amplification using yeast genomic DNA as the template and appropriate pairs of primers. A complete list of all oligo-primers used in this study is shown in Table 1. The resulting fragments were digested using the suitable restriction enzymes for which the restriction sites had been incorporated into the oligo-primers. The digested fragments were ligated into the vectors which were cut with the same enzymes and the whole ligation mixture was transformed into XL1-Blue cells (Stratagene). Several colonies of each plate were individually grown overnight in LB medium supplemented with the antibiotics and their plasmids were purified using Qiaprep Spin Miniprep Kit (Qiagen). Small amounts of the plasmids were subjected to enzymatic digestion followed by visualization on agarose gel to select for the positive constructs. Sequences of selected constructs were verified by DNA sequencing.

\section{Protein expression and purification}

In the case of single subunit proteins, each of the constructs was transformed into at least three different E.coli expression strains. The best expressing hosts were chosen based on two criteria: the expression level and the solubility of the protein after disruption of the cells. Both criteria were judged by eye on SDS-PAGE. T7-promoter driven protein expression was induced either using IPTG in $2 \times$ YT medium or by auto-induction (Studier, 2005).

Tif34 and Tif32 were expressed as N-terminally His-tagged proteins. In each case, cells were resuspended in buffer $\mathrm{A}$ (500 mM NaCl, $50 \mathrm{mM}$ HEPES, 5\% glycerol, $2 \mathrm{mM} \beta$-mercaptoethanol, $\mathrm{pH}$ 7.5) supplied with RNase A (Roche Applied Science), a mixture of protease inhibitors including aprotinin, leupeptin and pepstatin (ALP) and $20 \mathrm{mM}$ imidazole. Cells were lysed microfluidizer (Microfluidics, USA) and cell debris was removed by centrifugation at 30,000 $\times \mathrm{g}$ for 30 minutes on a JA-20 rotor (Beckman). Proteins were purified by applying the supernatants on a HISTrap column (GE Healthcare, Freiburg Germany) using Äkta Prime (GE Healthcare). After loading the sample, the column was washed with two column volumes of buffer $\mathrm{A}$. The protein was eluted by increasing the concentration of imidazole in several steps up to $500 \mathrm{mM}$. Tif32 was further purified by pooling and concentrating the fractions containing the least amount 
of impurities and applying it on a Superdex S-200 size exclusion column (GE Healthcare) equilibrated in $200 \mathrm{mM} \mathrm{NaCl}, 20 \mathrm{mM}$ Tris-HCl, 5\% glycerol and $2 \mathrm{mM}$ DTT at pH 8 . The leftmost fractions of the eluted peak which had the least amount of degradation were merged, concentrated and flash-frozen. The N-terminal His tag of Tif34 was removed using TEV protease while dialyzing overnight against dialysis200 buffer (200 mM NaCl, $30 \mathrm{mM}$ HEPES (pH 7.5), $5 \%$ glycerol and $2 \mathrm{mM} \beta$-mercaptoethanol). The residual His-tagged protein as well as the TEV protease was removed by a second step of Ni-NTA affinity purification using the same buffer as dialysis 200 buffer but with $150 \mathrm{mM} \mathrm{NaCl}$. The flow-through of the column was collected, concentrated and applied to a Superdex S-75 column (GE Healthcare) equilibrated in GF100 buffer (100 mM NaCl, $10 \mathrm{mM}$ HEPES (pH 7.5) and $2 \mathrm{mM}$ DTT). Fractions containing pure protein were pooled, concentrated and flash-frozen.

Tif35 and Nip1 were expressed as GST-fusion proteins. Cell resuspension and lysis were carried out essentially as that of Tif32 with the difference that no imidazole was added. The supernatant after centrifugation was applied to a GSTrap column (GE Healthcare) equilibrated in lysis buffer. After binding of the proteins, the column was washed with two column volumes of the lysis buffer and the fusion protein was eluted by washing the column with elution buffer (lysis buffer plus $30 \mathrm{mM}$ reduced glutathione). In both cases the GST tags were removed by treating the eluants with PreScission protease (GE Healthcare) in a 1:100 weight ratio of protease:protein while being dialyzed against dialysis200 buffer. The cleaved GST, PreScission protease and uncleaved proteins were removed by a second step of GSH sepharose. The flow-through of the GSTrap column containing the cleaved protein was collected, concentrated and applied to either a Superdex S-200 (for Nip1/3c) or S-75 (for Tif35/3g) equilibrated with GF150 buffer (150 mM $\mathrm{NaCl}, 10 \mathrm{mM}$ HEPES (pH 7.5), 5\% glycerol and $2 \mathrm{mM}$ DTT). Fractions containing pure protein were merged, concentrated and flash-frozen.

Prt1 ${ }^{181 \mathrm{C}}$ was expressed as an N-terminally His-tagged protein and purified in a way similar to Tif34. Hcr1/3j was purified as previously described (Khoshnevis, Neumann, and Ficner 2010).

To purify the complex of Tif32/Prt1/Nip1, each of the components were expressed individually as mentioned in the table 2.2. Cell pellets were suspended in buffer $\mathrm{B}(500 \mathrm{mM} \mathrm{NaCl}, 50 \mathrm{mM}$ HEPES, $5 \%$ glycerol, $2 \mathrm{mM} \beta$-mercaptoethanol, $0.05 \%$ tween-20, $1 \mathrm{mM} \mathrm{MgCl} 2$ and $0.5 \mathrm{mM}$ ATP) supplied with RNase A and Complete Protease Inhibitor Cocktail (Roche) and mixed prior to the lysis. Lysis was performed using a microfluidizer. Cell debris was removed by centrifugation at $30,000 \times \mathrm{g}$ for 30 minutes. Complex was purified by applying the supernatants 
on a GSTrap column, followed by washing with two column-volumes of buffer B, one columnvolume of buffer B-ATP/ $\mathrm{Mg}^{2+}$ (buffer B with $5 \mathrm{mM}$ ATP and $10 \mathrm{mM} \mathrm{MgCl}_{2}$ ) and again two column-volumes of buffer B. The complex was eluted by applying buffer B containing $30 \mathrm{mM}$ reduced glutathione instead of ATP and $\mathrm{MgCl}_{2}$. The GST tag was removed using PreScission protease while dialyzing against dialysis 300 buffer (the same as dialysis 200 but with $300 \mathrm{mM}$ $\mathrm{NaCl}$ ). The complex was further purified by removal of the GST, PreScission protease and noncleaved protein using a second GST affinity purification. The flow-through of the second GSTrap column was collected and subjected to a Ni-NTA affinity column against the His-tag of Tif32. The HISTrap column was washed with Wash200 buffer (200 mM NaCl, $30 \mathrm{mM}$ HEPES, $5 \%$ glycerol, $2 \mathrm{mM} \beta$-mercapto ethanol) containing $20 \mathrm{mM}$ imidazole and the bound proteins were eluted using a step gradient of the same buffer with increasing concentration of imidazole upto $500 \mathrm{mM}$. The elution peak was analyzed on SDS-PAGE and fractions containing the most pure complex were merged, concentrated and applied to a Superdex S-200 column equilibrated with buffer GF150. Fractions containing all three proteins were pooled, concentrated and flashfrozen.

\section{In vitro reconstitutions}

Tif34/Tif35/Prt ${ }^{181 \mathrm{C}}$ was reconstituted by mixing the three proteins in a molar ratio of 1:1.5:2. eIF3 $^{\text {rec }}$ (Tif32/Prt1/Nip1/Tif34/Tif35) was reconstituted by mixing the complex of Tif32/Prt1/Nip1 with Tif34 and Tif35 in a molar ratio of 1:2:2 to make sure that there is no unbound Tif32/Prt1/Nip1 complex. All in vitro complex reconstitutions were carried out in GF150 buffer at $4^{\circ} \mathrm{C}$.

\section{Isothermal titration calorimetry}

Tif34, Tif35 and Prt1 ${ }^{181 \mathrm{C}}$ were extensively dialyzed against ITC buffer (150 mM NaCl, $10 \mathrm{mM}$ HEPES pH 7.5, 5\% glycerol) and concentrated to $110,5.2$ and $10 \mu \mathrm{M}$, respectively. The experiment was performed on a VP-ITC calorimeter (Microcal). $20 \mu \mathrm{L}$ aliquots of Tif34 were injected into the cell containing either Tif35 or Prt ${ }^{181 \mathrm{C}}$ every 30 seconds, during which the titration peak returned to the base line. Separately, seven injections of the same concentration of Tif34 into the buffers were performed under the same conditions to determine the heat of dilution. The titration data were analyzed using the ORIGIN software to calculate the thermodynamics parameters.

\section{Interaction studies using analytical gel filtration}


Interaction studies were performed on analytical Superdex S-75 (10/300) or S-200 (10/300) columns (GE Healthcare). In each case a total amount of at least $50 \mu \mathrm{g}$ protein in the maximum volume of $500 \mu \mathrm{L}$ was loaded on the column to make the detection easy while preventing the broadening of the peak.

\section{Limited proteolysis}

Eight different proteases (thermolysin, elastase, GluC, aminopeptidase, carboxypeptidase, trypsin, chymotrypsin and proteinase K; Roche, Germany) were mixed with the indicated proteins or protein complexes in a weight ratio of 1:500. Samples were taken at different time points (1', 5', 15', 1 hour, 2 hours and 1 day) and analyzed by SDS-PAGE. The stable protein fragments/subcomplexes were tested for stability/integrity by analytical gel filtration. Borders of stable truncations were determined using Mass Spectroscopy.

\section{Native eIF3 and yeast ribosome purification}

Native preparations of yeast eIF3 (eIF3 ${ }^{\text {nat }}$ ) as well as $40 \mathrm{~S}$ and $60 \mathrm{~S}$ ribosomal subunits were carried out as previously described (Acker et al. 2007).

\section{Ribosome binding studies}

To study the interaction of eIF $3^{\text {rec }}$ to the ribosome, the complex was first reconstituted, mixed with Hcr1/3j and 40S subunit and applied on a $4 \%$ native acrylamide/poly-acrylamide gel as previously described (Acker et al. 2007). The gel was blotted on the nitrocellulose membrane and immunostained against the His-tag on Tif32/3a with horse-radish peroxidase conjugated antibody.

\section{Preparation and initial structural analysis of eIF3}

For single particle EM, 0.5-1 nmole of the freshly prepared complex was subjected to $10-30 \%$ glycerol gradient centrifugation and 0-0.1\% glutaraldehyde. Samples were prepared from the peak eIF3-containing fractions. Negative-staining was carried out with uranyl formate (Kastner et al. 2008). Images were recorded at $200 \mathrm{kV}$ and a magnification of 119,000 fold on a CM200 FEG electron microscope (Philips/FEI, Eindhoven, The Netherlands) equipped with a coarsened $4 \mathrm{k} \times 4 \mathrm{k}$ CCD camera operated with $2 \times$ pixel binning (F415 TVIPS, Gauting, Germany). 31,359 particles were selected manually. The untilted single particle images were aligned using exhaustive multireference alignment based on resampling to polar coordinates (Sander et al. 2003) and subjected to multivariate statistical analysis (MSA) and hierarchic ascendant 
classification, generating classes with 15-40 members on average in the context of the software IMAGIC-5 (van Heel et al. 1996). This procedure was iteratively repeated until the result was stable. In the final iteration, the aligned single particle images were divided into 1000 classes. Projection angle was assigned to class averages by angular reconstitution and subsequently the 3D structure was computed by an "exact fit" back-projection algorithm.

\section{Fluorescent labeling of Tif32/3a and transient kinetic studies}

Stopped-flow measurements were performed using the SX-20 MV stopped-flow apparatus (Applied Photophysics, Leatherhead, UK) and rapidly mixing equal volumes (60 $\mu 1$ each) of reactants at $20^{\circ} \mathrm{C}$. The working concentrations for Tif32 ${ }^{*} / \mathrm{Nip} 1$ complex formation were $0.1 \mu \mathrm{M}$ and $0.3 \mu \mathrm{M}$, respectively. For the chase experiments to study the binding of eIF3 to the $40 \mathrm{~S}$ subunit, $0.125 \mu \mathrm{M}$ Tif32 ${ }^{*}$ was mixed with Nip1, Prt1 ${ }^{181 \mathrm{C}}$ and Hcr1 in a 1:1.2:1.2:2.4 ratio. In case needed, $0.2 \mu \mathrm{M}$ of $40 \mathrm{~S}$ subunit was added. The complex was chased with $30 \mu \mathrm{M}$ of unlabeled Tif32. Alexa488 was excited at $465 \mathrm{~nm}$ and fluorescence emission was measured after passing cut-off filters (Schott AG, Mainz, Germany) KV500. Time courses (1,000 data points each, acquired in logarithmic sampling mode) were measured at pseudo-first-order condition and were evaluated by fitting an exponential function $F=F_{\infty}+A \times \exp \left(-k_{\text {app }} \times t\right)$, with a time constant $\left(k_{\text {app }}\right)$, the amplitude of the signal change $(A)$, the final signal $\left(F_{\infty}\right)$ and the fluorescence signal at time $\mathrm{t}(F)$. In case needed, extra exponential terms were added to the function. Calculations were performed using Prism (Graphpad Software, La Jolla, CA, USA). 
Table 2. 1. List of the oligo-primers used in this work. In each case, the forward primers used for cloning of a certain protein or fragment are mentioned first (denoted F) followed by the corresponding reverse primers (denoted R).

\begin{tabular}{|c|c|c|}
\hline Tif32 & F-Smal & $5^{\prime}$ T CCC CCG GGT ATG GCC CCC CCA CCA TTC 3' \\
\hline & R-Xhol & $5^{\prime}$ CCG CTC GAG TTA CCT GCC CCC CTT GGC $3^{\prime}$ \\
\hline $\begin{array}{l}\text { Tif32 } \\
578\end{array}$ & R-Xhol & $\begin{array}{l}5^{\prime} \text { CCG CTC GAG TTA CTT TTC CAT GTA TGA AGC ATT } \\
\text { GTT } 3^{\prime}\end{array}$ \\
\hline $\begin{array}{l}\mathrm{Tif}_{49} 2^{1-} \\
494\end{array}$ & R-Xhol & $\begin{array}{l}5^{\prime} \text { CCG CTC GAG TTA ATC TTT AGC GAA TGT AAC CTT } \\
\text { AGC } 3^{\prime}\end{array}$ \\
\hline Nip1 & F-Smal & $\begin{array}{l}5^{\prime} \text { T CCC CCG GGT ATG TCC CGT TTC TTT TCG TCT } \\
\text { AAT TAC } 3^{\prime}\end{array}$ \\
\hline $\operatorname{Nip}^{240-C}$ & F-BamHI & $5^{\prime}$ CCG GGA TCC ATT TCT TCG TCT CAA GGC AAT G 3' \\
\hline \multirow[t]{2}{*}{$\mathrm{Nip1}^{247-\mathrm{C}}$} & F-BamHI & $5^{\prime}$ CCG GGA TCC GAC CAA GCG GTA CAA GAA G $3^{\prime}$ \\
\hline & R-Xhol & $5^{\prime}$ CCG CTC GAG TCA ACG ACG ATT TGA TGG TGG G 3' \\
\hline Prt1 & F-Ndel & $\begin{array}{l}5^{\prime} \text { GG GAA TTC CAT ATG AAA AAT TTT CTT CCA CGC } \\
\text { ACA TTG AAA A } 3^{\prime}\end{array}$ \\
\hline \multirow[t]{2}{*}{$\operatorname{Prt1}^{670-\mathrm{C}}$} & F-BamHI & $5^{\prime}$ CCG GGA TCC AAC GCC GAA AGG AAA AAG GTT A $3^{\prime}$ \\
\hline & R-Xhol & $\begin{array}{l}5^{\prime} \text { CCG CTC GAG TTA TTC GAC CTT TTC CTT TGT TTC } \\
\text { TTC C } 3^{\prime}\end{array}$ \\
\hline \multirow[t]{2}{*}{$\operatorname{Prt1}^{181-\mathrm{C}}$} & F-Ndel & $\begin{array}{l}5^{\prime} \text { GG GAA TTC CAT ATG CCT ACA TTC GTT CCA TCT } \\
\text { AGT } 3^{\prime}\end{array}$ \\
\hline & R-Xhol & $\begin{array}{lllllllll}5^{\prime} & \text { CCG } & \text { CTC GAG TTC GAC } & \text { CTT } & \text { TTC } & \text { CTT } & \text { TGT } & \text { TTC TTC } \\
3 & & & & & & & \end{array}$ \\
\hline \multirow[t]{2}{*}{ Tif34 } & F-Ndel & $\begin{array}{l}5^{\prime} \text { GG GAA TTC CAT ATG AAG GCT ATC AAA TTA ACA } \\
\text { GGT CAT G } 3^{\prime}\end{array}$ \\
\hline & R-Xhol & $\begin{array}{l}5^{\prime} \text { CCG CTC GAG TTA ATT AGC TTC TTG CAT GTG CTC } \\
\text { TTT A } 3^{\prime}\end{array}$ \\
\hline \multirow[t]{2}{*}{ Tif35 } & F-Smal & $5^{\prime}$ T CCC CCG GGT ATG AGT GAA GTT GCA CCA GAG 3' \\
\hline & R-Xhol & $\begin{array}{l}5^{\prime} \text { CCG CTC GAG CTA TTC CTT AAC CTT AGG TTT GGA } \\
\mathrm{C}^{\prime}\end{array}$ \\
\hline $\begin{array}{l}\text { Tif35 } \\
150\end{array}$ & F-BamHI & $\begin{array}{lllllll}5^{\prime} & \text { CCG GGA TCC GAT GGA TCT AGA TCT ATC ATT ACA } \\
\text { TA } & 3^{\prime}\end{array}$ \\
\hline $\begin{array}{l}\operatorname{Tif3}_{150}^{14-} \\
150\end{array}$ & R-Xhol & $\overline{5^{\prime}}$ CCG CTC GAG TTA CTT CTC TTC ACT GGC GGC $3^{\prime}$ \\
\hline \multirow[t]{2}{*}{ Hcr1 } & F-BamHI & $5^{\prime}$ CCG GGA TCC ATG TCT TGG GAC GAC GAA G $3^{\prime}$ \\
\hline & R-Xhol & $\begin{array}{l}5^{\prime} \text { CCG CTC GAG TTA CAT AAA GTC GTC ATC ACC GAA } \\
3^{\prime}\end{array}$ \\
\hline
\end{tabular}


Table 2. 2. The list of expression vector and strains used in this study. This table lists the optimal combination of the vector, position and type of the tag, as well as the expression strain which was used to obtain the highest amount of soluble protein.

\begin{tabular}{|c|c|c|c|}
\hline Tif32 & pET28b & $\mathrm{N}-\mathrm{His}$ & Rosetta2 \\
\hline Tif32 $2^{1-494}$ & pET28b & $\mathrm{N}$-His & Rosetta2 \\
\hline Tif32 $2^{1-57} 8$ & pET28b & $\mathrm{N}$-His & Rosetta2 \\
\hline Nip1 & pGEX-6P-1 & N-GST & $\mathrm{Bl} 21$ (DE3) \\
\hline $\mathrm{Nip} 1^{240-\mathrm{C}}$ & pGEX-6P-1 & N-GST & $\mathrm{B} \mid 21$ (DE3) \\
\hline $\mathrm{Nip}^{247-\mathrm{C}}$ & pGEX-6P-1 & N-GST & $\mathrm{Bl} 21$ (DE3) \\
\hline Prt1 & pET22b & $\mathrm{C}-\mathrm{His}$ & Rosetta2 \\
\hline Prt1 ${ }^{181-C}$ & pET15b & $\mathrm{N}-\mathrm{His}$ & Rosetta2 \\
\hline Prt1 ${ }^{670-C}$ & pGEX-6P-1 & N-GST & Rosetta2 \\
\hline Tif34 & pET15b & $\mathrm{N}-\mathrm{His}$ & $\mathrm{B} \mid 21$ (DE3) \\
\hline Tif35 & pGEX-6P-1 & N-GST & Rosetta2 \\
\hline Tif35 $5^{14-150}$ & pGEX-6P-1 & N-GST & $\mathrm{B} \mid 21$ (DE3) \\
\hline Hcr1 & pGEX-6P-1 & N-GST & Rosetta2 \\
\hline
\end{tabular}

\subsection{Acknowledgment}

We would like to thank J.R. Lorsch and A.G. Hinnebusch for providing us with the yeast strains for the purification of ribosome and native eIF3, respectively. We are thankful to B. Schmidt for the analysis of eIF3 fragments by Mass spectrometry. We express our gratitude to A. Dickmanns and T. Monecke for critically reading the manuscript. 


\section{Chapter $3 \bullet$ Crystal Structure of Yeast elF3b-}

\section{RRM}

This manuscript has originally been published in PLOS ONE

\section{Crystal Structure of the RNA Recognition Motif of Yeast Translation Initiation Factor elF3b reveals differences to human elF3b.}

Sohail Khoshnevis, Piotr Neumann, Ralf Ficner

Department of Molecular Structural Biology, Institute for Microbiology and Genetics, Georg-August-University Göttingen, Germany

${ }^{\ddagger}$ To whom correspondence should be addressed: E-mail: rficner@uni-goettingen.de PLoS ONE, 2010, 5(9): e12784.

Received July 4, 2010; Accepted August 19, 2010; Published September 16, 2010

Copyright: (c) 2010 Khoshnevis et al. This is an open-access article distributed under the terms of the Creative Commons Attribution License, which permits unrestricted use, distribution, and reproduction in any medium, provided the original author and source are credited.

Authurs contributions:

SK: design of the experiments, cloning, purification, crystallization, structure determination, ITC, RNA binding assay, data analysis and writing the manuscript. PN: structure determination and writing the manuscript. RF: design of the experiments, data analysis and writing the manuscript. 


\section{Abstract}

Background: The multi-subunit eukaryotic initiation factor3 (eIF3) plays a central role in the initiation step of protein synthesis in eukaryotes. One of its large subunits, eIF3b, serves as a scaffold within eIF3 as it interacts with several other subunits. It harbors an RNA Recognition Motif (RRM), which is shown to be a non-canonical RRM in human as it is not capable to interact with oligonucleotides, but rather interacts with eIF3j, a sub-stoichiometric subunit of eIF3.

Principal Finding: We have analyzed the high-resolution crystal structure of the eIF3b RRM domain from yeast. It exhibits the same fold as its human ortholog, with similar charge distribution on the surface interacting with the eIF $3 \mathrm{j}$ in human. Thermodynamic analysis of the interaction between yeast eIF3b-RRM and eIF3j revealed the same range of enthalpy change and dissociation constant as for the human proteins, providing another line of evidence for the same mode of interaction between eIF3b and eIF3j in both organisms. However, analysis of the surface charge distribution of the putative RNA-binding $\beta$-sheet suggested that in contrast to its human ortholog, it potentially could bind oligonucleotides. Three-dimensional positioning of the so called "RNP1" motif in this domain is similar to other canonical RRMs, suggesting that this domain might indeed be a canonical RRM, conferring oligonucleotide binding capability to eIF3 in yeast. Interaction studies with yeast total RNA extract confirmed the proposed RNA binding activity of yeast eIF3b-RRM.

Conclusion: We showed that yeast eIF3b-RRM interacts with eIF3j in a manner similar to its human ortholog. However, it shows similarities in the oligonucleotide binding surface to canonical RRMs and interacts with yeast total RNA. The proposed RNA binding activity of eIF3b-RRM may help eIF3 to either bind to the ribosome or recruit the mRNA to the 43S preinitiation complex.

\subsection{Introduction}

Translation initiation in eukaryotes is intimately regulated by a set of proteins known as eukaryotic initiation factors (eIFs), which exploit diverse functions from positioning of the initiator tRNA in the ribosomal P-site to the recognition of mRNA (Sonenberg and Hinnebusch 2009). The largest of these factors, eIF3, is a multi-subunit complex consisting of five stoichiometric subunits in yeast (eIF3a, b, c, g and i). Mammalian eIF3 has thirteen subunits, five of which are homologous to the yeast subunits of eIF3, hence believed to form the core of eIF3 and fulfill its essential functions (Hinnebusch 2006). The sixth subunit of eIF3 in yeast, 
eIF3j (Hcr1), is a loosely associated subunit and is suggested to play a role in recruitment of eIF3 to the ribosome (Nielsen et al. 2006). In addition to its role in translation initiation, eIF3j is also involved in the biogenesis of ribosomal RNA (Valásek et al. 2001). It has also been suggested that eIF3j might serve a cross-talk function between translation initiation and termination by its interaction with translation termination factor eRF1 as well as eIF3 (Khoshnevis et al. 2010). eIF3j interacts with the C-terminal domain (CTD) of eIF3a as well as the RNA recognition motif of eIF3b (Valásek et al. 2001). Solution structure of human eIF3bRRM with a short peptide of eIF3j revealed the binding site for eIF3j to be located in a cleft between two helices of the RRM, distant from the exposed surface of the $\beta$-sheet which in other RRMs binds to the target RNA or DNA (Elantak et al. 2010). Interestingly, human eIF3b-RRM was shown to be a non-canonical RRM due to the high distribution of the negative charges around the canonical oligonucleotide binding site which makes it very unlikely to be able to accommodate any oligonucleotide (Elantak et al. 2007). Comparing the predicted isoelectric points of human and yeast eIF3b-RRM shows that in contrast to negatively charged human eIF3b-RRM, the yeast homolog is highly positively charged. Here, by using X-ray crystallography we studied the structure of yeast eIF3b-RRM and showed that it corresponds to the canonical RNA recognition motif. This domain also exhibited binding to the yeast total RNA, further confirming the proposed function. Nonetheless, determination of its specific physiological target remains out of the scope of this work. We further studied the thermodynamics of the interaction between eIF3b-RRM and eIF3j in yeast and showed that similar to human, this interaction is enthalpy driven. Given the similarity in surface charge distribution at the eIF3j binding site between yeast and human eIF3b-RRM as well as the conservation of the eIF3j peptide interacting with this domain, we propose that yeast applies the same mode of interaction with eIF3j as human.

\subsection{Results and Discussion}

\section{Structure of Yeast eIF3b-RRM}

Two different truncations of the N-terminal domain of yeast eIF3b were designed based on homology to the published structure of its human ortholog (Elantak et al. 2007). Crystals of the shorter truncation (rsidues 76-161) diffracted to $2.6 \AA$ A. However, they were not easily reproducible as bunches of needles were obtained instead. The second construct encompassing residues 76-170 was designed to have an extra helix according to the secondary structure predictions. Highly reproducible crystals were obtained in space group $\mathrm{P} 2{ }_{1} 2_{1} 2_{1}$ with two 
molecules per asymmetric unit which diffracted to $1.25 \AA$. Crystallographic phases were obtained by molecular replacement using the structure of the sex-lethal RRM domain as search model. Data processing and refinement statistics are provided in Table 1. The structure is composed of four $\beta$-strands and two $\alpha$-helices which are arranged in a $\beta-\alpha-\beta-\beta-\alpha-\beta$ sequential order. The last $\beta$-strand is followed by a short $\alpha$-helix which connects this domain to the rest of the protein (Figure 3.1.A). This $\alpha$-helix is absent in the NMR structure of human eIF3b-RRM. The two yeast eIF3 $b^{76-170}$ monomers occupying the asymmetric unit are related by a two-fold non-crystallographic symmetry axis, forming a closely packed dimer (Figure 3.1.B).

A

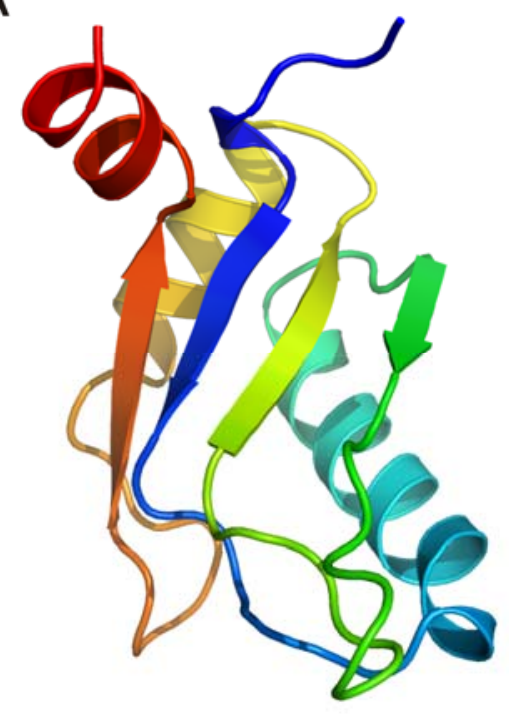

B

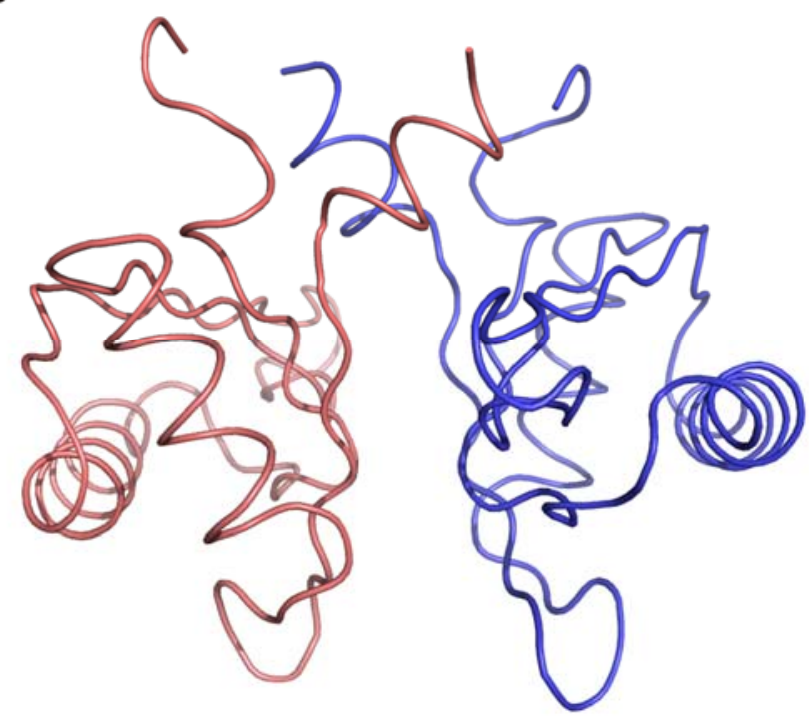

Figure.3. 1. Overall structure of yeast elF3b-RRM. (A) Overall fold of the yeast elF3b-RRM showing the canonical $\beta-\alpha-\beta-\beta-\alpha-\beta$ fold which at the $C$-terminus is followed by an extra helix which connects this domain to the rest of the protein ( $\mathrm{N}$ - and $\mathrm{C}$-termini are colored blue and red, respectively). (B) Relative orientation of two monomers in the asymmetric unit. Two monomers are held in place by interactions between residues in their $\beta$-sheets as well as the c-terminal helices. A 180 o non-crystallographic symmetry axis exists between two monomers.

Analysis of the structure by PISA (http://www.ebi.ac.uk/msd-srv/prot_int/pistart.html) (Krissinel and Henrick 2007) shows that $14.8 \%\left(1805 \AA^{2}\right)$ of the total surface of the two molecules (12193 $\AA^{2}$ ) gets buried upon the dimer formation. Two monomers in the asymmetric unit are held in place by interactions between their $\beta$-sheets and between each $\beta$-sheet and the extended $\alpha$-helix of the neighboring chain. Comparison of the relative orientation of the monomers between eIF3b-RRM ${ }^{76-170}$ and eIF3b-RRM ${ }^{76-161}$ showed that chain $B$ in eIF3b-RRM ${ }^{76-161}$ has been displaced by $\sim 9 \AA$ relative to the same chain in eIF3b-RRM ${ }^{76-170}$ (Figure 3.2). Therefore this dimer is most probably an artifact of the crystallization and not the functional unit of the protein 
in the solution. This is in agreement with the gel-filtration profile of the protein showing that it eluted from the column at a volume corresponding to the size of about $10 \mathrm{kDa}$ (data not shown).

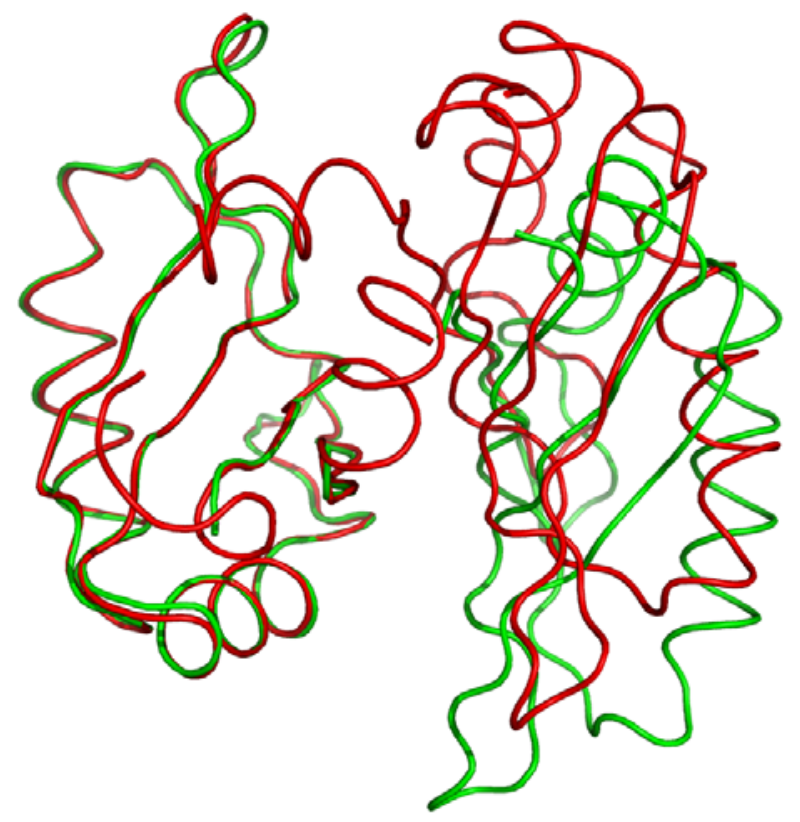

Figure.3.2. Different dimer formation between two different truncations of yeast elF3b-RRM. Superimposition of the chains $A$ of the yeast $R_{R M}{ }^{76-161}$ (red) and $R_{R M}{ }^{76-170}$ (green) indicates the difference in the relative orientation of the dimers between two molecules, indicating that the formation of the dimer is probably an artifact of the crystallization.

\section{Yeast eIF3b-RRM Interacts with eIF3j in a Similar Fashion to Its Human Homolog}

Analysis of the surface charge distribution of the yeast eIF3b-RRM revealed a high degree of similarity to its human homolog with the surface made up of two $\alpha$-helices packed against the $\beta$ sheet is rich in positively charged residues, creating an overall basic region distal to the accessible surface of the $\beta$-sheet (Figure 3.3.A-B). The negatively-charged eIF3j peptide which interacts with human eIF3b-RRM (Elantak et al. 2010) reveals amino acid conservation in yeast (Figure 3.4) which suggests the same mode of interaction between yeast eIF3b-RRM and eIF3j. Superposition of reported structure with that of human in complex with a short peptide of eIF3j (PDB code $2 \mathrm{KRB}$ ) suggests that lysines 97, 105, 142, 147 and arginine 148 provide a positively charged surface for the accommodation of the negatively charged peptide of eIF3j. Another line of evidence for the same mechanism of the interaction between yeast $3 b-R R M$ and $3 \mathrm{j}$ comes from the analysis of the thermodynamics of their interaction using isothermal titration calorimetry (ITC), showing that the $K_{d}$ of their interaction lies in the $\mu \mathrm{M}$ range, similar to the reported value for the human proteins. The raw ITC data and the integrated areas under each peak as a function of molar ratio of eIF3b-RRM to eIF3j are plotted in the upper and lower 
panels of Figure 3.5, respectively. The interaction is characterized by a large negative enthalpy change $\left(\Delta H=-9278 \pm 217.3 \mathrm{kcal} . \mathrm{mol}^{-1}\right)$, indicating that similar to the human $3 \mathrm{~b}$-RRM, it is predominantly mediated through electrostatic interactions (Elantak et al. 2007). The binding curve is sigmoidal and best fitted to a single binding site model with $\sim 1: 1$ stoichiometry, yielding a dissociation constant $\left(K_{d}\right)$ of $7.5 \pm 0.5 \mu \mathrm{M}$.

A

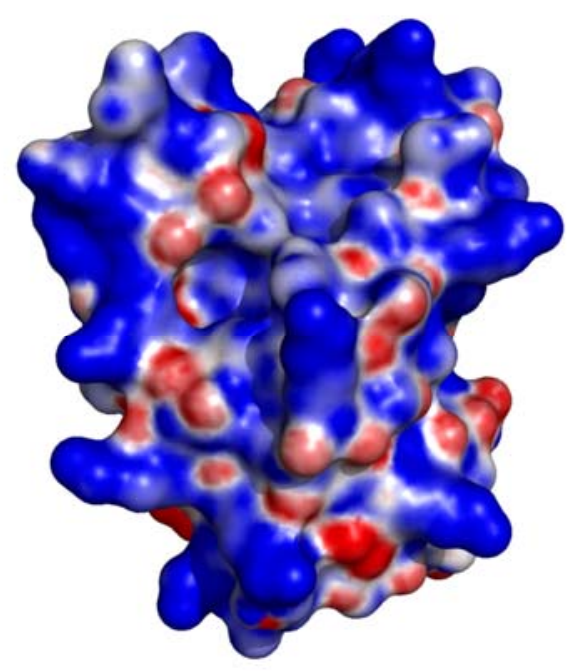

C

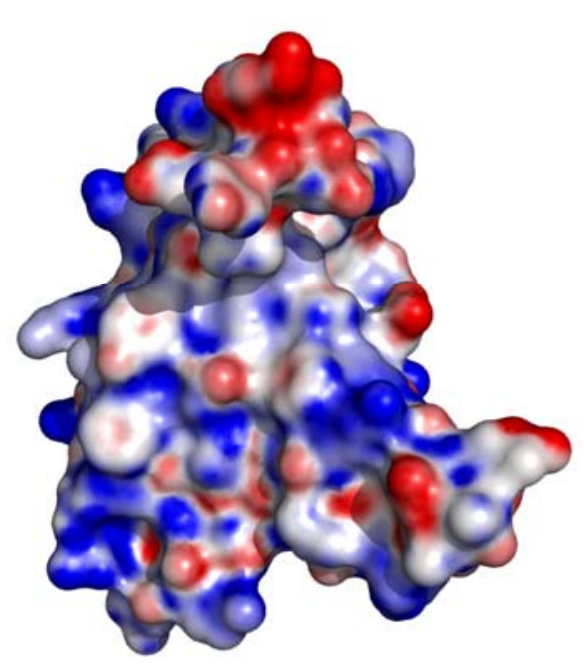

\section{B}

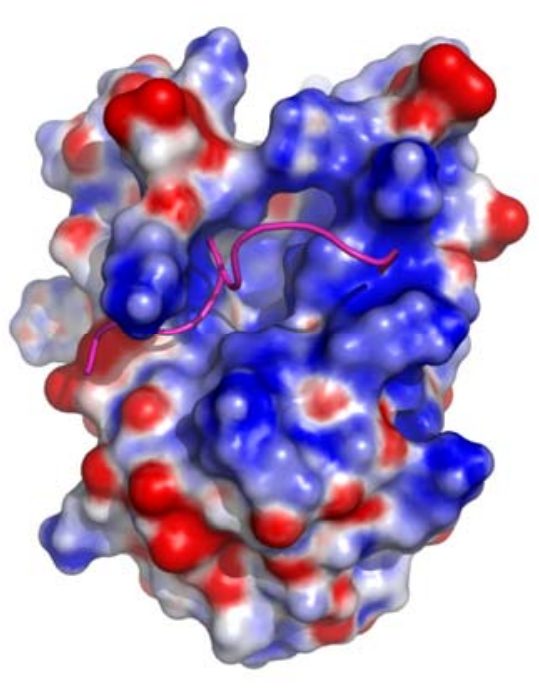

D

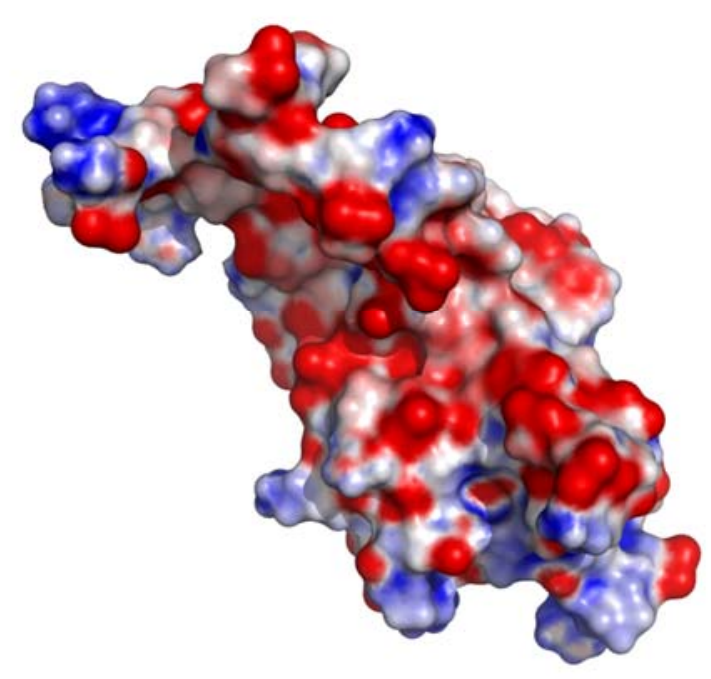

Figure.3.3. Surface charge distribution of yeast and human elF3b-RRM. (A) Surface charge distribution of yeast elF3b-RRM viewed from the $\alpha$-helical side of the domain (distal to the oligonucleotide binding side) showing the accumulation of positive charges which would provide a suitable binding site for the negatively charged peptide of elF3j. (B) Surface charge distribution of human elF3b-RRM from the same view as (A). The short negatively charged peptide of elF3j (magenta) sits in a basic cleft on elF3b-RRM. (C). Surface charge distribution on the nucleotide binding side of the yeast elF3b-RRM indicates the dominance of the positive over negative charges. This suggests that this motif can accommodate oligonucleotides. (D). Surface charge distribution on the nucleotide binding side of the humanelF3bRRM. Accumulation of acidic side-chains leaves no room for accommodation of any oligonucleotide. 


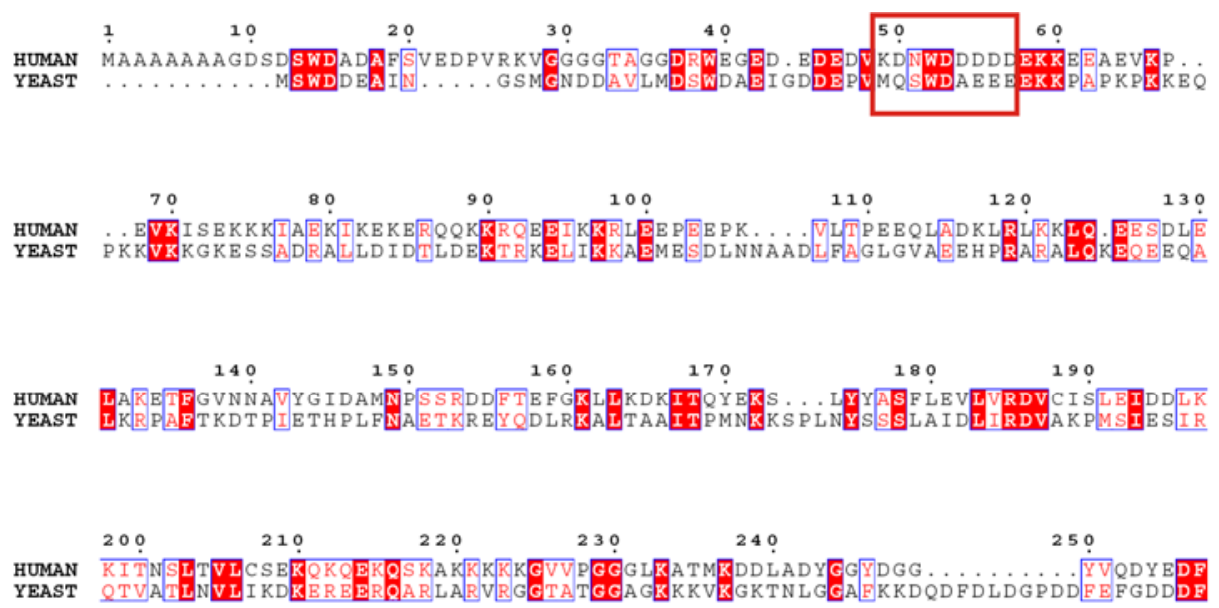

$\underset{\text { HEAST }}{\text { HUMAN }}$

Figure.3.4. Sequence-based alignment of human and yeast elF3j. The boxed sequence is the fragment which was solved in complex with human elF3b-RRM (PDB code 2KRB). Conservation of the sequence of this region suggests the same mode of binding between yeast eIF3j and elF3b-RRM. The alignment and the color representation were performed using ClustalW2 (www.ebi.ac.uk/Tools/clustalw2) (Larkin et al. 2007) and ESPript 2.2 (www.espript.ibcp.fr/ESPript) (Gouet et al. 2000) respectively.

\section{Oligonucleotide Binding Potentials of Yeast eIF3b-RRM}

Analysis of the surface charge distribution of the yeast eIF3b-RRM revealed that in contrast to the human eIF3b-RRM, the solvent accessible surface of the $\beta$-sheet (distal to its surface which is packed against $\alpha$-helices) is either neutral or positively charged (Figure 3.3.C). This surface contains the "RNP1" motif, known for the canonical RRMs, comprised of the consensus sequence of [RK]-G-[FY]-[GA]-[FY]-[ILV]-X-[FY] (Cléry et al. 2008). Superposition of the structures of the monomer of yeast eIF3b-RRM with six canonical RRMs (PDB codes 1B7F, 2AD9, 2KH9, 2RQC, 3D2W and 2UP1) (Handa et al. 1999; Oberstrass et al. 2005; MartinTumasz et al. 2010; Tsuda et al. 2009; Kuo et al. 2009; Ding et al. 1999) bound to oligonucleotide (either RNA or DNA) suggests the conservation of these residues in three dimensions, which together with the appropriate charge distribution make should allow for binding to oligonucleotides (Figure 3.6). Surprisingly, the human homologue of this protein also shows three-dimensional conservation of this motif, however due to the accumulation of negatively charged residues on the surface of its $\beta$-sheet, it is not capable of accommodating an oligonucleotide (Figure 3.3.D). 


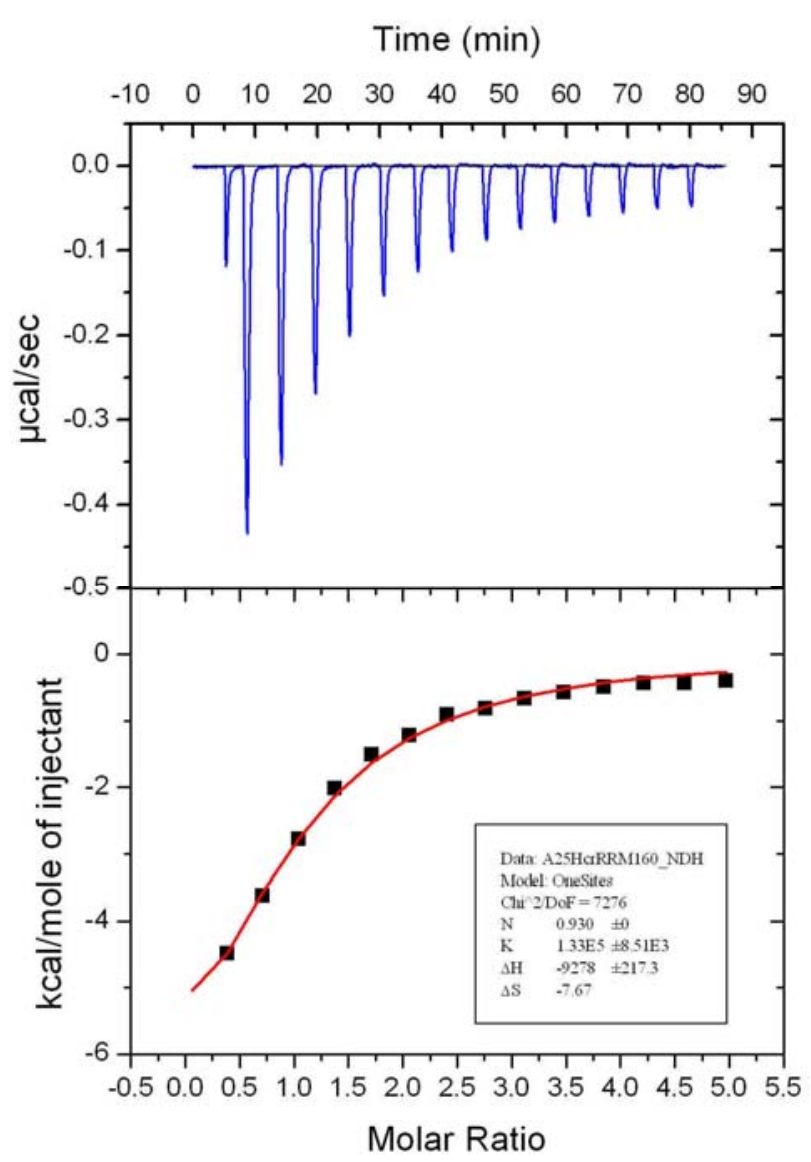

Figure.3.5. Isothermal calorimetric titration of elF3j with elF3b-RRM. The upper panel shows raw data of heat effect (in $\mu \mathrm{cal} \cdot \mathrm{s}^{-1}$ ) of $20-\mu \mathrm{l}$ injections of $229 \mu \mathrm{M}$ elF3b-RRM into $1.5 \mathrm{ml}$ of $10 \mu \mathrm{m}$ elF3j performed at $300 \mathrm{~s}$ intervals. The lower panel shows the fitted binding isotherms. The data points were obtained by integration of heat signals plotted against the molar ratio of elF3b-RRM to elF3j in the reaction cell. The solid line represents a calculated curve using the best fit parameters obtained by a nonlinear least squares fit.

Superpositioning of structures of yeast eIF3b-RRM with hnRNP A1 (UP1) complexed with single-stranded telomeric DNA (PDB code 2UP1) suggests it to bind in the same manner to an oligonucleotide, with Phe 126 stacking against the sugar pocket of one nucleotide and Phe 128 against the base of the next nucleotide, whilst the Lys 124 would neutralize the backbone phosphate (Figure 3.7.B). Interestingly, comparison of the dimer of eIF3b-RRM with hnRNP A1 (UP1) shows that Tyr 158, Asp 162 and Phe166 of one monomer interact with the RNP1 motif of the second monomer in the crystal, mimicking the presence of the oligonucleotide (Figure 3.7.A and C). 

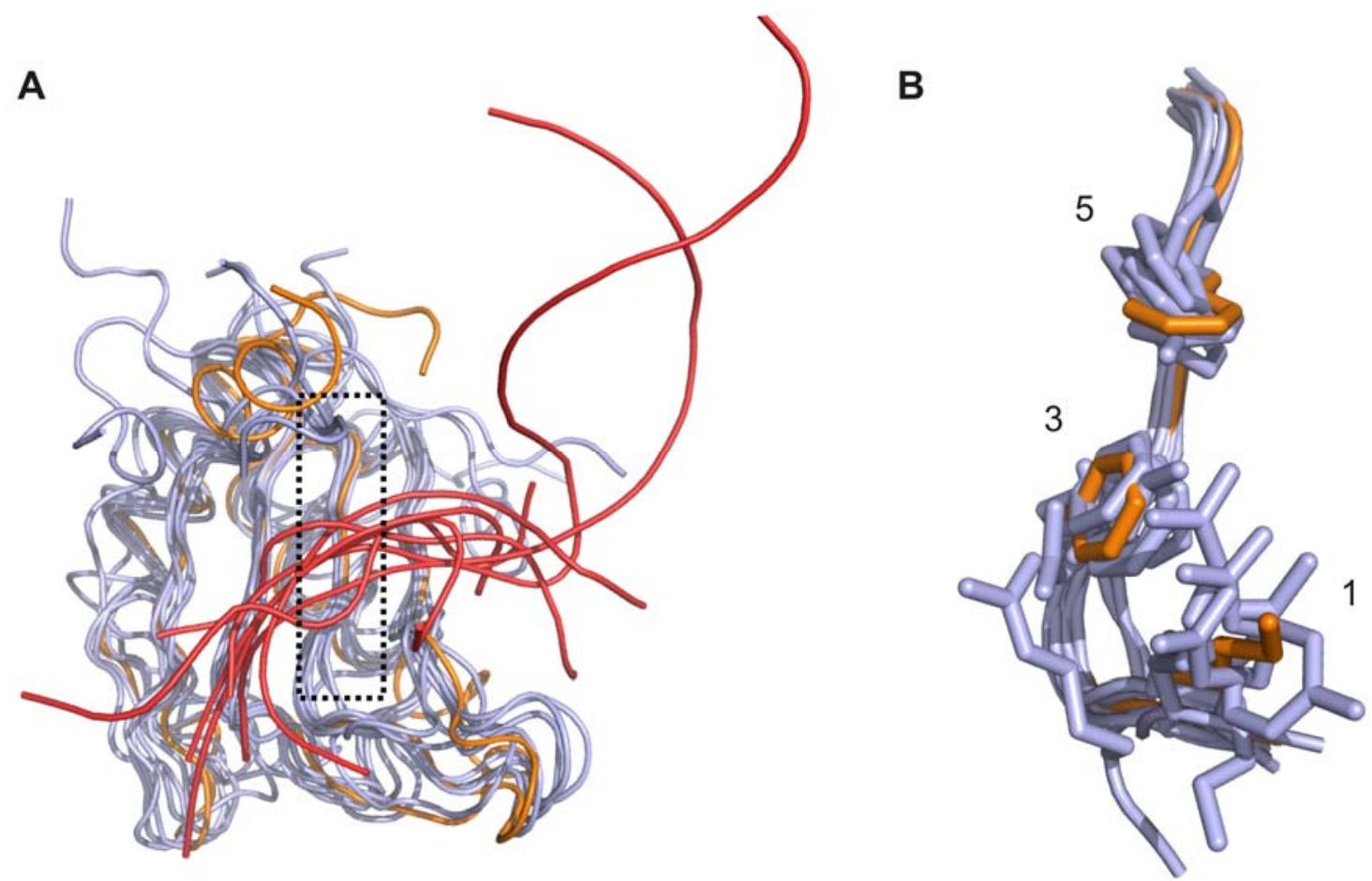

Figure.3.6. Superimposition of yeast elF3b-RRM with several canonical RRMs bound to oligonucleotides from PDB. (A) Six different canonical RRMs (PDB codes 1B7F, 2AD9, 2KH9, 2RQC, 3D2W and 2UP1, all in grey) superimposed with yeast elF3b-RRM (orange). As shown, oligonucleotides (red loops) occupy more or less the same position on the solvent exposed side of the $\beta$-sheet. (B) Three dimensional conservation of the elements of RNP1 (the black box on the panel A). The numbers correspond to the position of the amino acids in the motif $[\mathrm{RK}]_{1}-\mathrm{G}_{2}-[\mathrm{FY}]_{3}-[\mathrm{GA}]_{4}-[\mathrm{FY}]_{5}-[\mathrm{ILV}]_{6}-\mathrm{X}_{7}-[\mathrm{FY}]_{8}$.

Another motif known as "RNP2" with the sequence [ILV]-[FY]-[ILV]-X-N-L also plays a role in the interaction with the oligonucleotide, though it is not as conserved as the RNP1. For instance, in the structure of sex-lethal protein in complex with RNA (PDB code 1B7F) (Handa et al. 1999) the first RRM shows deviation from this consensus sequence, whereas the second RRM harbors the exact consensus motif. Therefore deviation of this motif from the consensus in yeast eIF3bRRM does not exclude its oligonucleotide binding capacities. Nonetheless, in the superimposed structure, Asn82 which belongs to RNP2 motif in an alternative conformation is close to the putative position of the base of another nucleotide, which in the crystal is occupied by Phe156 from another monomer in the asymmetric unit (Figure 3.7.C). 

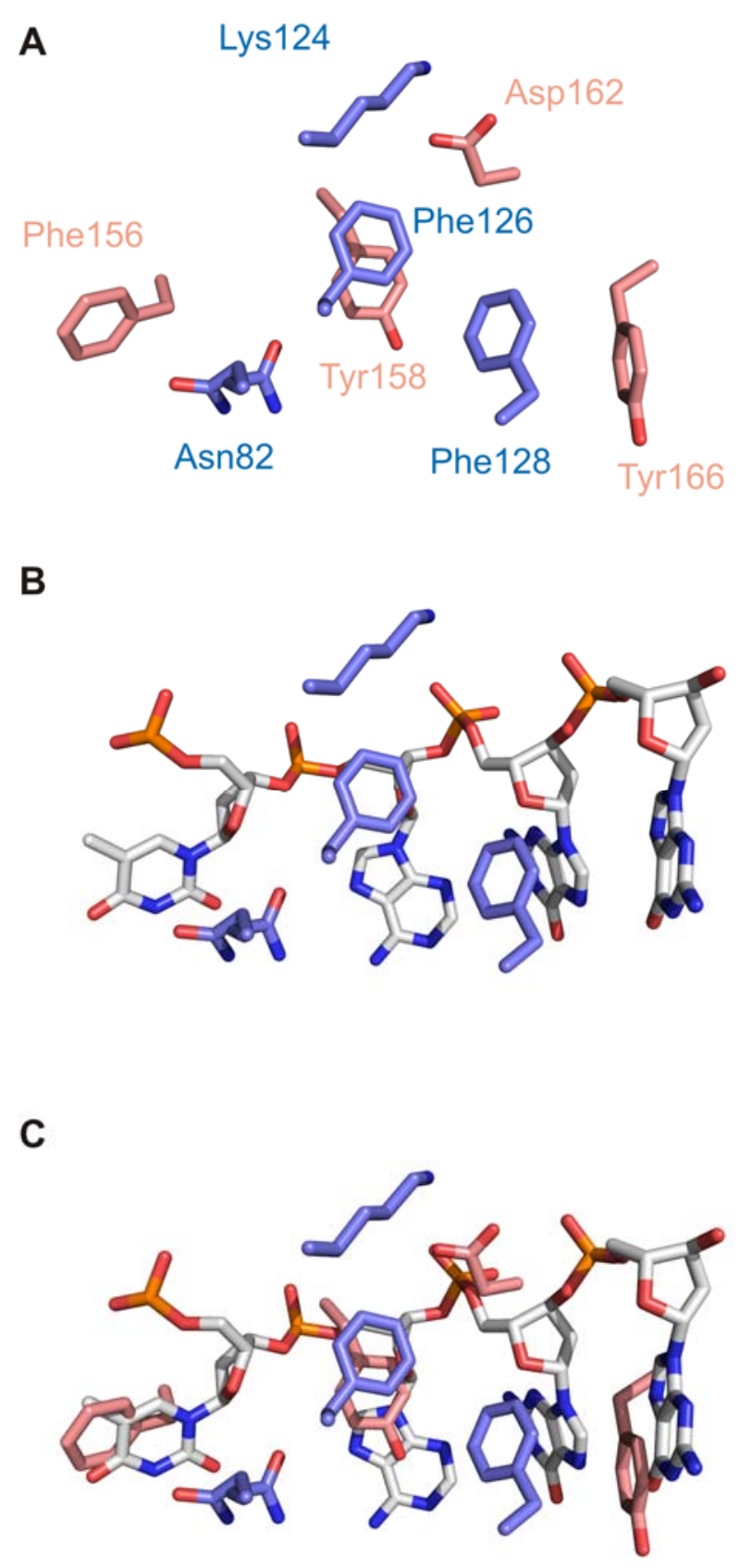

Figure.3. 7. Critical interactions between two monomers in the asymmetric unit mimic the presence of an oligonucleotide. (A). The dimer of the $\mathrm{RRM}^{76-170}$ is held by several interactions, some of which involve components of the RNP1 (Lys 124, Phe 126 and Phe 128) or RNP2 (Asn 82). (B) Superposition of elF3bRRM with hnRNP A1 (UP1) in complex with single-stranded telomeric DNA (PDB code 2UP1) shows that different elements of RNP1 and RNP2 can interact with RNA bases (Asn82 in one of its alternative conformations and Phe128), sugar pocket (Phe126) or phosphate backbone (Lys124). (C) The interacting partners for the residues depicted in panel $B$ assume the position of certain elements of the docked oligonucleotide; e.g. Asp 162 sits where the negatively charged phosphate group would sit, Phe 156 and Phe 166 occupy the position of bases of the oligonucleotide and Tyr 158 mimics the sugar pocket.

To test whether the proposed RNA binding activity occurs in vitro, the protein was mixed with the weight excess of the yeast total RNA to saturate the protein with RNA as much as possible 
and studied the interaction at two different salt concentrations by EMSA on a $5 \%$ native PAGE. Due to the overall positive charge of the protein, the gel had to be run in a positive-to-negative direction to get it into the gel. Results showed that in comparison to a sharp single band for the RRM alone, the addition of RNA resulted in smearing of the band upwards, indicating the formation of several RNA-protein complexes. The smearing was more pronounced at $80 \mathrm{mM}$ than $160 \mathrm{mM}$ salt (Figure 3.8.A). To confirm these results, a filter binding assay was performed using the same complex preparations as for the EMSA but with the weight excess of the protein over the RNA to bind as much RNA as possible to the membrane for the sake of better detection. Results indicated higher binding of the yeast total RNA to the membrane in the presence of the RRM. Here, we also observed more binding at lower salt concentration (Figure 3.8.B). Taken together, the results indicate the interaction between yeast eIF3b-RRM with its total RNA.

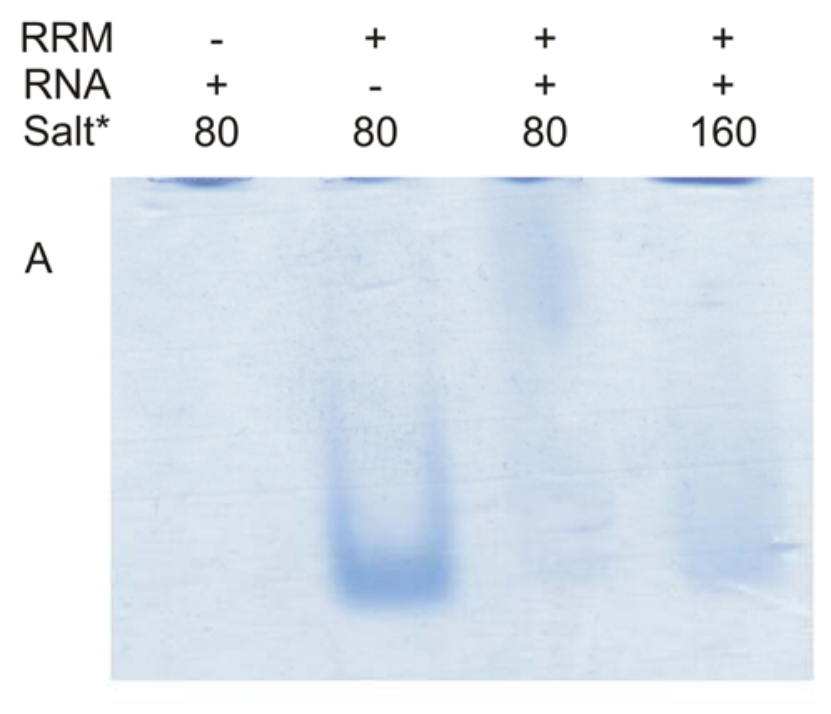

B

Figure.3. 8. Binding studies between yeast IF3b-RRM and total RNA extract. (A). Electrophoretic mobility shift assay between RRM and total RNA indicates the upwards smearing of the RRM band upon interaction with RNA. The effect is more pronounced at lower salt concentration. (B). Filter binding assay confirms the results obtained by EMSA. Drops of filter binding assay are aligned under the corresponding lanes of EMSA. * stands for mM.

Although these results propose an RNA binding function of yeast eIF3b-RRM, determination of its exact RNA target is out of the scope of this work. However, it is tempting to speculate that the RNA binding ability conferred to eIF3 by this domain might serve to either recruit it to the ribosome via interactions with certain areas of the ribosomal RNA or facilitates its interaction 
with the mRNA, hence promoting the recruitment of the mRNA to the 43S pre-initiation complex. Although the human homolog of this domain has no RNA binding capability, this function may be fulfilled by additional components of human eIF3 which are missing in yeast.

\subsection{Materials and Methods}

\section{Cloning}

Yeast eIF3b-RRM spanning amino acid 76 to 161 or 170 and Hcrlwere PCR amplified from yeast genomic DNA using following primers: 5, CCGGGATCCGATCAGT ACATCGTCGTTAATG 3' and 5' CCGCTCGAGTTAGTCGTCAGAATTATATCTTT CAACA 3' or 5' CCGCTCGAGTTATTTCATAGTATAAAGAAACAAACGATGT 3' for RRM ${ }^{76-170}$ and RRM $^{76-161}$, respectively, and CCGGGATCCATGTCTTGGGACGA CGAAG and CCGCTCGAGTTACATAAAGTCGTCATCACCGAA for eIF3j. Resulting fragments were digested by BamH1 and Xho1 and ligated into the pGEX-6P-1 vector (GE Healthcare) which was digested with the same restriction enzymes. The ligation mixtures was then transformed into XL1-Blue cells (Stratagene) and plated on LB plates containing ampicillin. Positive transformants were determined by DNA sequencing and transformed into strain Rosetta2 (DE3) (Novagen).

\section{Protein expression and purification}

For large-scale protein expression, $10 \mathrm{~mL}$ of preculture of each protein was prepared by resuspending several colonies of Rosetta2 (DE3) cells harboring either eIF3b-RRM or eIF3j in pGEX-6P-1 into LB medium supplemented with appropriate antibiotics at $37^{\circ} \mathrm{C}$. Next day, 500 $\mathrm{mL} 2 \mathrm{XYT}$ media containing chloramphenicole and ampicilline was inoculated with $5 \mathrm{~mL}$ of each preculture and incubated at $37^{\circ} \mathrm{C}$ at $220 \mathrm{rpm}$. The protein expressions were induced with $0.2 \mathrm{mM}$ IPTG at $\mathrm{OD} \sim 0.5$ and cells were subsequently transferred to $17^{\circ} \mathrm{C}$. After 20 hours, cells were harvested and the resulted cell pellets were shock-frozen in liquid nitrogen. For protein purification, cells were resuspended in lysis buffer $(300 \mathrm{mM} \mathrm{NaCl}, 50 \mathrm{mM}$ HEPES pH 7.5, $5 \%$ Glycerol and $2 \mathrm{mM} \beta$-mercaptoethanol). Prior to the lysis, a protease mixture containing aprotinin, leupeptin and pepstatin was added to the suspension. Cell rupture was carried out by passing the cells six times through the microfluidizer (Microfluidics, Newton, US). Cell debris was removed by centrifugation at $30,000 \mathrm{~g}$ for 30 minutes on a JA-20 rotor (Beckman). The proteins were purified by applying the supernatants on a $5 \mathrm{~mL}$ GSTrap column (GE Healthcare) using Äkta prime (GE Healthcare). After loading the sample, the column was washed for two 
column volumes with wash buffer (2M LiCl, 50 mM HEPES pH 7.5, 5\% Glycerol, $2 \mathrm{mM} \beta$ mercaptoethanol) to remove any bound oligonucleotide and then equilibrated back in lysis buffer. GST-fusion protein was eluted by washing the column with elution buffer (the same as lysis buffer plus $30 \mathrm{mM}$ reduced glutathione). Fractions containing the fusion protein were pooled together and treated with PreScission protease (GE Healthcare) overnight to cleave off the GST. Fro RRM purification, this sample was concentrated the next day to $5 \mathrm{~mL}$ and loaded on a Superdex S-75 (26/60) column (GE Healthcare) pre-equilibrated in GF buffer (100 mM $\mathrm{NaCl}, 10 \mathrm{mM}$ HEPES pH 7.5, 2 mM DTT). Fractions containing eIF3b-RRM were merged together and concentrated to $12 \mathrm{mg} \mathrm{mL}^{-1}$ and flash frozen in liquid nitrogen. For Hcr1 purification, the cleaved GST and the PreScission protease were removed by a second step of glutathione affinity chromatography and the GST-free Hcr1 sample was loaded on a Superdex S75 (26/60) column pre-equilibrated in GF buffer. The eluant protein was concentrated to $17 \mathrm{mg}$ $\mathrm{mL}^{-1}$ and flash frozen in liquid nitrogen.

\section{Crystallization, data collection and structure determination}

Needle-shape crystals of eIF3b-RRM ${ }^{76-170}$ were obtained at $20^{\circ} \mathrm{C}$ using sitting drop method by mixing equal volumes of protein $\left(11 \mathrm{mg} \cdot \mathrm{mL}^{-1}\right)$ with $30 \%$ PEG 4000, $200 \mathrm{mM} \mathrm{Li}_{2} \mathrm{SO}_{4}$ and 100 $\mathrm{mM}$ HEPES $\mathrm{pH}$ 8. Crystals of good diffraction quality grew after three days to the final size of $100 * 20 * 10$ micrometer. Datasets were collected at the beamline ID23-2 at ESRF, Grenoble. The data set was processed in the space group P $2{ }_{1} 2_{1} 2_{1}$ using XDS (Kabsch 2010) and scaled to the final resolution of $1.25 \AA$. The phase problem was solved by molecular replacement using the crystal structure of Drosophila melanogaster sex-lethal protein (PDB code 3SXL) (Crowder et al. 1999) as the search model in Phaser (McCoy et al. 2007). The initial model was further built and improved manually in Coot (Emsley and Cowtan 2004) and subsequently subjected to iterative steps of refinement in Phenix (Adams et al. 2010) and manual model building in Coot.

Plate-shape crystals of eIF3b-RRM ${ }^{76-161}$ were obtained by mixing the same volume of the protein $\left(17 \mathrm{mg} \cdot \mathrm{mL}^{-1}\right)$ with the reservoir containing 33\% PEG4000 and $0.1 \mathrm{M}$ Na-citrate (pH 5.6) in sitting drop plates at $20^{\circ} \mathrm{C}$. Good diffracting crystals appeared after two weeks. A dataset was collected on the home source beamline equipped with MAR345dtb detector mounted on a Micromax 007 generator operating with a copper target at $1.5417 \AA$. The dataset was indexed, scaled and reduced using XDS and SCALA (Evans 2006) in the space group P $2{ }_{1} 2_{1} 2_{1}$ to the final resolution of $2.6 \AA$. The structure of eIF3b-RRM ${ }^{76-170}$ monomer was used as the search model in Phaser. This model was subsequently subjected to iterative steps of refinement in Phenix and 
manual model building in Coot.

Both structures are deposited at the Protein Data Bank with the accession codes 3NS5 (eIF3b$\mathrm{RRM}^{76-161}$ ) and 3NS6 (eIF3b-RRM ${ }^{76-170}$ ).

\section{Isothermal titration calorimetry}

Hcr1 and eIF3b-RRM ${ }^{76-170}$ were extensively dialyzed against ITC buffer (200 mM NaCl, $10 \mathrm{mM}$ HEPES pH 7.5, 5\% Glycerol) and concentrated to 10 and $229 \mu \mathrm{M}$, respectively. The experiment was performed on a VP-ITC calorimeter (Microcal). $20 \mu \mathrm{L}$ aliquots of RRM were injected into the cell containing Hcrl every 40 second, during which the titration peak returned to the base line. Separately, seven injections of the same concentration of RRM into the buffers were performed under the same conditions to determine the heat of dilution. The titration data were analyzed using the ORIGIN software to calculate the thermodynamics parameters.

\section{RNA binding assays}

Yeast total RNA was extracted from strain InviScI (Invitrogen) using Nucleospin RNA II kit (Macherey-Nagel, Germany). $5 \%$ Acrylamide gel was made as described previously (Acker et al. 2007). To allow the detection by Coomassie Blue $2 \mu \mathrm{g}$ of the protein was applied to $5 \mu \mathrm{g}$ of the RNA in a reconstitution buffer (Acker et al. 2007) at the final volume of $10 \mu \mathrm{L}$. The salt concentration of the buffer was changed to obtain the final salt concentration of either 80 or 160 $\mathrm{mM}$. As the control, two samples, one only with RRM and the other only with RNA were prepared in the final salt concentration of $80 \mathrm{mM}$. After 15 minutes of incubation at $25^{\circ} \mathrm{C}$, samples were mixed with $2 \mu \mathrm{L}$ of $6 \mathrm{X}$ loading dye $(50 \%$ sucrose, $0.02 \%$ bromophenol blue, 0.02 $\%$ xylene cyanol) and loaded on the native gel. The gel was run at 7-10 $\mathrm{W}$ in the cold room for 30 minutes in a positive-to-negative direction. Filter binding assay was performed by mixing 10 $\mu \mathrm{g}$ of the protein with $5 \mu \mathrm{g}$ of the RNA in a final volume of $10 \mu \mathrm{L}$ in the same buffers as for the native gel. After 15 minutes of incubation at $25^{\circ} \mathrm{C}$, the whole sample was loaded as a drop on a nitrocellulose membrane connected to the vacuum. Each drop was washed two times with reconstitution buffer and stained with GelRed (BIOTIUM, USA). 
Table 3. 1. Summary of the data collection and structure refinement

\begin{tabular}{|c|c|c|}
\hline & elF3b-RRM ${ }^{76-170}$ & elF3b-RRM $^{76-161}$ \\
\hline \multicolumn{3}{|l|}{ A. Data collection } \\
\hline Space group & $\mathrm{P} 22_{1} 2_{1}$ & $\mathrm{P} 22_{1} 2_{2}$ \\
\hline \multicolumn{3}{|l|}{ Unit cell parameter } \\
\hline$a(\AA)$ & 43.71 & 30.28 \\
\hline$b(\AA)$ & 51.75 & 68.14 \\
\hline$c(\AA)$ & 77.1 & 81.89 \\
\hline Resolution $(\AA)$ & $30-1.25(1.35-1.25)$ & $34-2.6(2.73-2.6)$ \\
\hline Observed reflections & 256057 (41259) & $24819(3276)$ \\
\hline Unique reflections & 48971 (8169) & $5632(771)$ \\
\hline Completeness (\%) & $99.6(99.4)$ & $99.1(94.6)$ \\
\hline$|/ \sigma|$ & $12.42(4.23)$ & $10.8(4.3)$ \\
\hline$R_{\text {merge }}{ }^{a}$ & $8.1(50.3)$ & $11.1(32.0)$ \\
\hline Monomers in $\mathrm{AU}^{\mathrm{b}}$ & 2 & 2 \\
\hline \multicolumn{3}{|l|}{ B. Refinement } \\
\hline Resolution $(\AA)$ & 1.2 & 2.6 \\
\hline$R_{\text {work }}{ }^{c}$ & 14.16 & 22.5 \\
\hline$R_{\text {free }} d^{d}$ & 16.75 & 27.25 \\
\hline $\begin{array}{l}\text { Number of protein atoms } \\
\text { in the } A U\end{array}$ & 1538 & 1392 \\
\hline Number of solvent atoms & 301 & 50 \\
\hline $\begin{array}{l}\text { Number of ligand atoms } \\
\text { and ions }\end{array}$ & 20 & 0 \\
\hline \multicolumn{3}{|l|}{ Mean B-value $\left(\AA^{2}\right)$} \\
\hline Protein & 11.473 & 24.732 \\
\hline Solvent & 23.931 & 16.929 \\
\hline Ligands and ions & 30.611 & \\
\hline \multicolumn{3}{|l|}{ Rmsd from ideal } \\
\hline Bond length $(\AA)$ & 0.008 & 0.001 \\
\hline Bond angles $\left({ }^{\circ}\right)$ & 1.247 & 0.480 \\
\hline \multicolumn{3}{|l|}{ Ramachandran plot $(\%)^{\mathrm{e}}$} \\
\hline Favoured & 100 & 98.86 \\
\hline Outlier & 0 & 0 \\
\hline Allowed & 0 & 1.14 \\
\hline PDB codes & 3 NS6 & $3 N S 5$ \\
\hline
\end{tabular}

Values in brackets refer to the highest resolution shell.

$\left.{ }^{a} R_{\text {merge }}=\Sigma_{h} \Sigma_{1}\left|l_{i h}\left\langle I_{h}\right\rangle\right| / \Sigma_{h} \Sigma_{1}<l_{h}\right\rangle$, where $\left.<I_{h}\right\rangle$ is the mean of the observations $l_{i h}$ of reflection $h$.

${ }^{\mathrm{b}} \mathrm{AU}$ stands for asymmetric unit.

${ }^{c} R_{\text {work }}=\Sigma_{\text {hkl }}|| F_{\text {obs }}|-| F_{\text {calc }}|| / \Sigma_{\text {hkl }}\left|F_{\text {obs }}\right|$, where $F_{\text {obs }}$ and $F_{\text {calc }}$ are the observed and calculated structure factors, respectively.

${ }^{\mathrm{d}} \mathrm{R}$ factor calculated for $5 \%$ randomly chosen reflections not included in the refinement.

${ }^{\mathrm{e}}$ The geometry of the models was analyzed by Molprobity (Davis et al. 2004). 
3. Crystal Structure of yeast eIF3b-RRM

\subsection{Acknowledgment}

We are grateful to the staff at the beamline ID23-2 at ESRF for the assistance during X-ray diffraction data collection. We thank Achim Dickmanns for critically reading the manuscript. 


\section{Chapter $4 \bullet$ Rli1 functions in translation}

\section{termination}

This manuscript has originally been published in EMBO Reports

\section{The iron-sulfur protein Rli1 functions in translation termination}

Sohail Khoshnevis ${ }^{1,2}$, Thomas Gross ${ }^{2,3}$, Carmen Rotte ${ }^{2,1}$, Claudia Baierlein $^{3}$, Ralf Ficner ${ }^{1,4}$ and Heike Krebber ${ }^{3,4,5}$

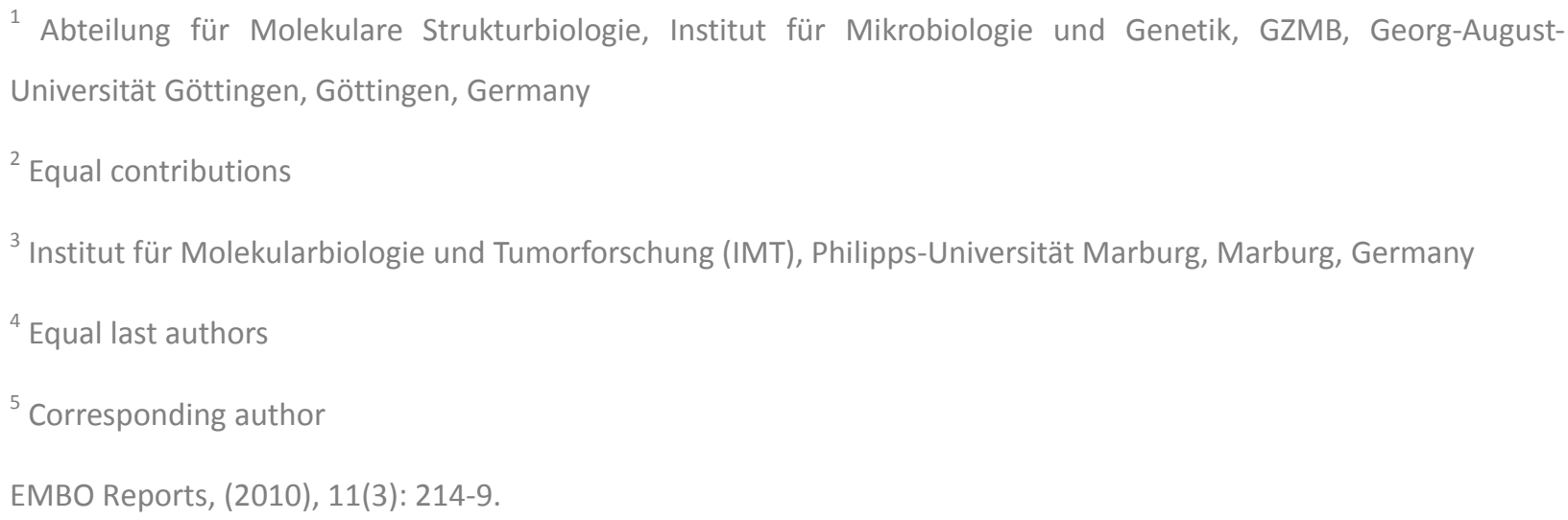

SK: cloning, protein purification, pull-down experiments, drop tests, data analysis and manuscript preparation. TG: drop-tests, dual reporter assay, IP, data analysis. CR: cloning, protein purification, Y2H, pull-down experiments, data analysis and manuscript preparation. CB: drop-tests. RF: data analysis and manuscript preparation. HK: data analysis and manuscript preparation. 


\begin{abstract}
The iron-sulfur (Fe/S) containing RNase L inhibitor (Rli1) is involved in ribosomal subunit maturation, transport of both ribosomal subunits into the cytoplasm and translation initiation through interaction with the eIF3-complex. Here we present a novel function for Rli1 in translation termination. By co-immunoprecipitation experiments we show that Rli1 physically interacts with the translation termination factors eRF1/Sup45 and eRF3/Sup35 in Saccharomyces cerevisiae. Genetic interactions were uncovered between a strain depleted for Rli1 and sup35-21 or sup45-2. Further, we show that down regulation of the RLII expression leads to defects in the recognition of a stop codon, as seen in mutants of other termination factors. In contrast, RLII overexpression partially suppresses the readthrough defects in sup45-2. Interestingly, although we find that the Fe/S cluster is not required for the interaction of Rli1 with eRF1 or its other interacting partner Hcrl from the initiation complex eIF3, it is required for its activity in translation termination, as a Fe/S cluster mutant of $R L I 1$ can not suppress the readthrough defects of sup 45-2.
\end{abstract}

\title{
4.1. Introduction
}

Translation is well-conserved in eukaryotes and can be separated into three distinct steps: initiation, elongation and termination (Kapp and Lorsch 2004b). Initiation involves the assembly of translation-competent ribosomes on mRNAs and depends on eukaryotic initiation factors (eIFs) that stimulate ribosome loading. One of these, eIF3 has 6 subunits in Saccharomyces cerevisiae (13 in humans), one of which is termed Hcrl. It functions by stimulating the formation of the $43 \mathrm{~S}$ pre-initiation complex that binds to the mRNA and scans it to identify the AUG-Start codon (Hinnebusch 2006). Interestingly, eIF3 also regulates the dissociation of the ribosome after translation termination (Pisarev et al. 2007).

Upon synthesis of the polypeptide chain in translation elongation, termination takes place. The process is mediated by recognition of a stop codon via the transfer RNA (tRNA)-mimicking protein eRF1 (Sup45 in S. cerevisiae) and subsequent hydrolysis of the ester bond connecting the polypeptide chain and the tRNA, stimulated by the GTPase-activity of eRF3 (Sup35 in $S$. cerevisiae) (Jacobson 2005). Further, the DEAD-box RNA helicase Dbp5 has recently been identified to function in translation termination (Gross et al. 2007). It assists eRF1 in stop codon recognition and controls the subsequent eRF1-eRF3 interaction through its dissociation from eRF1 (Gross et al. 2007). The activity of Dbp5 is stimulated by its co-factor Gle1 and the small molecule inositol hexakisphosphate ( $\mathrm{InsP}_{6} / \mathrm{IP6}$ ) (Bolger et al. 2008). Interestingly, Gle1 also 
influences translation initiation, as it genetically and physically interacts with subunits of the initiation factor eIF3 and mutants show defects in translation initiation (Bolger et al. 2008).

Here we have identified the essential iron-sulfur containing RNase L inhibitor (Rli1), which belongs to the family of ATP-binding cassette (ABC) proteins, as a novel translation termination factor. Rli1 requires the mitochondrial and cytosolic $\mathrm{Fe} / \mathrm{S}$ protein biogenesis machineries for its assembly and mutations in critical cysteine residues of Rlil abolish its association with Fe/S clusters, leading to the loss of cell viability (Kispal et al. 2005; Lill, 2009). Rli1 associates with polyribosomes (Dong et al. 2004) and with Hcr1, which is proposed to play a dual role in rRNA processing as well as in translation initiation. Rli1 mutants are impaired in pre rRNA processing and defective in the export of both ribosomal subunits (Kispal et al. 2005; Yarunin et al. 2005). Further, evidence was provided that Rli1 is required for efficient formation and stabilization of 43S and 48S pre-initiation complexes (Dong et al. 2004). Rli1 associates with components of the eukaryotic translation initiation machinery, eIF2, eIF5 and, in particular, the translation initiation complex eIF3. Hcr1 was visibly enriched in Rli1p-TAP purifications (Yarunin et al. 2005). This, together with preliminary yeast-two-hybrid experiments (Kispal et al. 2005) suggest that Hcr1 and Rli1 may directly interact and that Hcr1 may link Rli1 to the eIF3 complex and translation initiation.

In human cells RLI1 was originally identified as an inhibitor of RNase L (Bisbal et al. 1995). RNase L was characterized as a protein that is activated via the interferon system upon viral infection (Jacobson 2005). Recently, an interaction of RNase L with eRF3 was identified and it was consequently shown that this interaction leads to an increased translational readthrough efficiency at premature termination codons and an increased +1 frameshifting efficiency, which might play an important role in the antiviral response (Jacobson 2005; Le Roy et al. 2005). Remarkably, Rlil is highly conserved from yeast to human, which can not be explained by a conserved function in viral defence.

In this study we have identified a novel function of the RNase L inhibitor Rli1 as a translation termination factor. We show physical and genetic interactions between Rli1 and both translation termination factors, eRF1 and eRF3. We further demonstrate that the second ABC domain of Rli1p is sufficient to mediate the interaction with both Hcr1 and eRF1. Moreover we find that a functional $\mathrm{Fe} / \mathrm{S}$ cluster is necessary for its role in proper recognition of a stop codon. 


\subsection{Results and Discussion}

\section{A two-hybrid screen identifies Sup45 as an Rli1 interacting protein}

In order to identify novel Rli1 interacting proteins a yeast two-hybrid screen was performed with full length $R L I 1$ fused to the DNA binding domain of the transcriptional activator GAL4 and a cDNA library, fused to the GAL4 activation domain. Approximately $\sim 5 \times 10^{5}$ yeast clones were screened and 55 plasmids were identified that gave strong two-hybrid interaction signals with the three reporter genes, $A D E 2, H I S 3$, and lacZ (Figure S4.1). Notably, among these 55 proteins, 32 were identified to encode for HCRl indicating a favored interaction between these two proteins. In addition, genes that encode for proteins involved in ribosome biogenesis (Rpl32, Dbp10, Rps20, Rps16), glucose (Pdc1, Std1, Tdh3) and amino acid metabolism (His3) and translation termination (Sup45) were identified. Given that ribosomal proteins are known false positive candidates in yeast-two-hybrid screens (Hengen 1997), and since we do not expect a direct functional role of Rli1 in glucose- or amino acid metabolism, we focused on Hcr1, Sup45, and Dbp10 for further characterization of their interaction with Rli1. While the interactions of both, Sup45 and Hcr1 with Rli1 were verified from both positions (used as a bait or as a prey), an interaction of Rli1 with Dbp10 however, could not be confirmed (data not shown). It will be interesting to characterize the remaining two-hybrid interactions in future experiments and to potentially uncover other important novel function for Rli1. The two-hybrid interaction of Rli1 and Hcrl is in agreement with earlier results suggesting a direct binding of these proteins (Kispal et al. 2005). In contrast, the two-hybrid interaction detected between Rli1 and Sup45 has never been identified before and thus both proteins might represent novel potential interaction partners. Interestingly, a two hybrid interaction with Sup35 was not identified. This may be due to the potential absence of the gene from the two hybrid library in the first place, or due to the possibility that the tagging of the protein prevented an interaction with Rli1.

\section{Rli1 physically interacts with both translation termination factors eRF1 and eRF3 in vivo}

To investigate a potential physical interaction between Rli1 and translation termination factors we performed interaction studies with both, eRF1 (Sup45) and eRF3 (Sup35). Wildtype yeast cells were transformed with a plasmid encoding HA-tagged Rli1. Cells were grown to the logarithmic growth phase, before co-immunoprecipitations were performed with Rli1-HA. All samples were treated with RNase to ensure that a direct protein-protein interaction was detected. The eluates were analyzed on SDS-PAGE and the proteins were visualized by immunostaining. As shown in figure 4.1.A, both translation termination factors were detected to interact with 
Rli1. To confirm these interactions, we also used TAP-tagged Sup45 and Sup35 to coimmunoprecipitate Rli1-HA (Figure 4.1.B). With both methods we found a physical interaction of Rli1 with both translation termination factors.

A

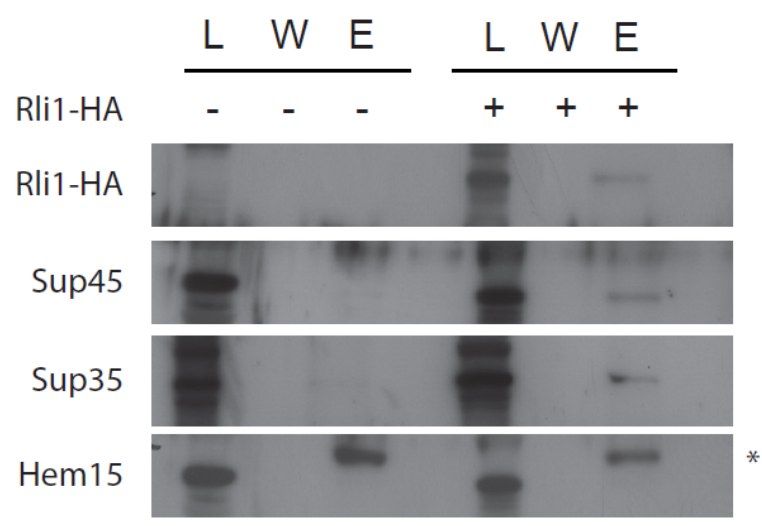

B

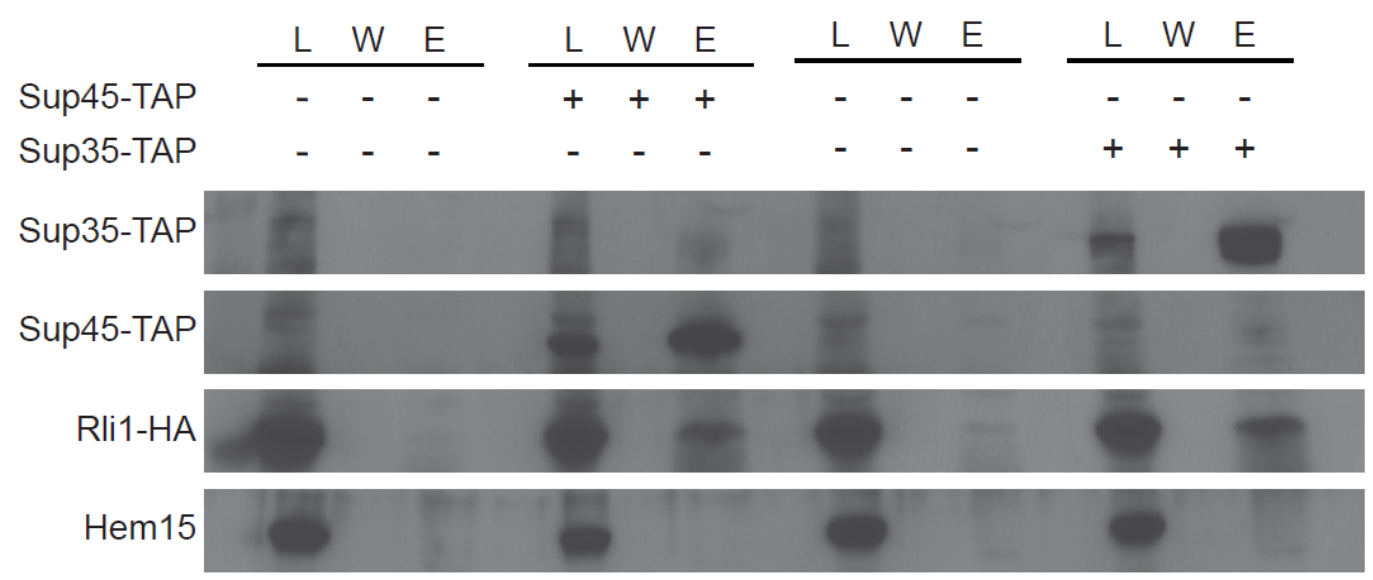

Figure 4. 1. Rli1 physically interacts with the translation termination factors eRF1/Sup45 and eRF3 Sup35. (A) Co-immunoprecipitation experiments were performed with HA-tagged Rli1 and Sup45 and Sup35 from log phase yeast cells. Lysates (L), wash fractions (W) and the eluates (E) are shown in western blot analyses. $10 \mu \mathrm{l}$ of each lysate and the complete eluates were loaded onto the gel. Each 200 $\mu \mathrm{l}$ eluate including the beads was finally resuspended in $20 \mu \mathrm{l}$ of sample buffer and entirely loaded, which represents 20 times the lysate. Antibodies against HA, Sup45, Sup35 and Hem15 (negative control) were used for detection. The bands visible in the Hem15 eluate fractions are caused by the presence of the cross-reacting antibody chains and are marked by an asterisk. (B) TAP-tagged SUP45 and SUP35 were purified from log phase yeast cells, carrying a plasmid encoding RLI1-HA. Wildtype cells expressing plasmid encoded RLI1-HA served as a negative control. Western blots of the lysates (L), wash fractions (W) and the eluates $(E)$ are shown in western blot analyses. The ratio of lysate to eluate is 1:20. Antibodies against $\mathrm{HA}$ and Hem15 (negative control) were used for detection. 


\section{Mapping of the Rli1 interaction domain}

To gain insights into the binding region of Rli1 that mediates the interaction with both, Sup45 and Hcr1, we performed additional two-hybrid analyses with truncated versions of the protein. While rlil mutants lacking the N-terminal Fe/S clusters, both $\mathrm{Fe} / \mathrm{S}$ clusters or the first $\mathrm{ABC}$ domain were able to interact with Sup45 and Hcr1, deletion mutants lacking the second Cterminal ATPase domain failed to interact with either Sup45 or Hcr1 (Figure 4.2.A). These results indicate that the $\mathrm{Fe} / \mathrm{S}$ clusters might not be required for the protein interactions. In contrast, we show that the second C-terminal ABC ATPase domain of Rli1 is crucial for the interaction between Rli1 and both, Sup45 and Hcr1 in two-hybrid analyses. From these data we can conclude that the second $\mathrm{ABC}$ domain might mediate the interaction of Rli1 with both, Hcr1 and Sup45.

Further, we confirmed the interactions between Rli1 and Hcr1 and Sup45 by in vitro binding experiments. Recombinant GST fusion of Rli1 and His-tagged Hcr1 and Sup45 were constructed. The GST-Rli1 fusion protein was co-expressed in E. coli with either His-Hcr1 or His-Sup45, respectively and purified on glutathione-Sepharose beads. After extensive washing, the beads were analyzed on SDS-PAGE and the proteins were visualized by immunostaining. While GST-Rli1 pulled down His-Hcr1 (Figure 4.2.B) and His-Sup45 (Figure 4.2.C), neither GST alone nor GST-PNC1, used as a negative control, were able to pull down His-tagged Hcr1. Similarly, GST-Rli1, but not GST alone or GST-Hcr1 interact with the His-tagged Sup45 protein. Moreover, binding of Sup45 and Hcr1 to Rli1 were still observed in the presence of higher salt concentration $(150 \mathrm{mM} \mathrm{NaCl})$, indicating a robust interaction of Rli1 with the translation factors. In addition, we conclude from these in vitro studies, that both Rli1-interactions are direct and do not require additional factors.

\section{Genetic interaction by partial repression of the Rli1 expression in combination with mutations in SUP45 and SUP35}

In order to investigate potential genetic interactions between $R L I 1$ and the termination factors, we used a strain, in which the $R L I 1$-expression can be regulated by the addition of doxocycline (tet:RLI1) (Kispal et al. 2005). Doxocycline treatment of tet:RLI1 cells, which completely represses the expression of $R L I 1$ is lethal (supplementary Figure S4.2), however, a partial suppression still allows growth of the yeast cells as shown with equal numbers of cells that were spotted in serial dilutions onto full medium (YPD) plates and plates containing different concentrations of doxocycline. As shown in figure 4.3.A, partial repression of $R L I 1$-expression 
in combination with sup45-2 is synthetically lethal, indicating a strong genetic interaction between RLI1 and SUP45. In contrast to that, the combination of down-regulated RLI1 and sup35-21 shows only a slight growth defect, which might indicate a more significant relationship between Rli1 and Sup45.

A

\begin{tabular}{|c|c|c|c|c|}
\hline \multicolumn{3}{|c|}{ Rli1 } & Hcr1 & Sup45 \\
\hline \begin{tabular}{|l|l|}
$\mathrm{Fe} / \mathrm{S}$ & $\mathrm{Fe} / \mathrm{S}$ \\
\end{tabular} & $\overline{A B C}$ & $A B C$ & + & + \\
\hline \multicolumn{3}{|c|}{\begin{tabular}{|l|l|}
$\mathrm{Fe} / \mathrm{S}$ & $\mathrm{Fe} / \mathrm{S}$ \\
\end{tabular}} & \multicolumn{2}{|c|}{ not expressible } \\
\hline $\mathrm{Fe} / \mathrm{S}$ & $\overline{A B C}$ & & - & - \\
\hline & $\overline{A B C}$ & $A B C$ & + & + \\
\hline & $\overline{A B C}$ & & - & - \\
\hline & & $\overline{A B C}$ & + & + \\
\hline
\end{tabular}

B
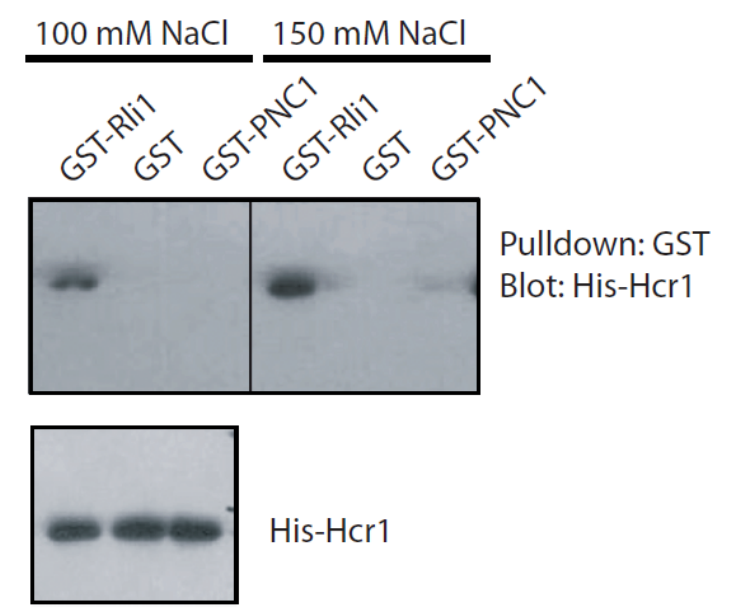

C

His-Hcr1

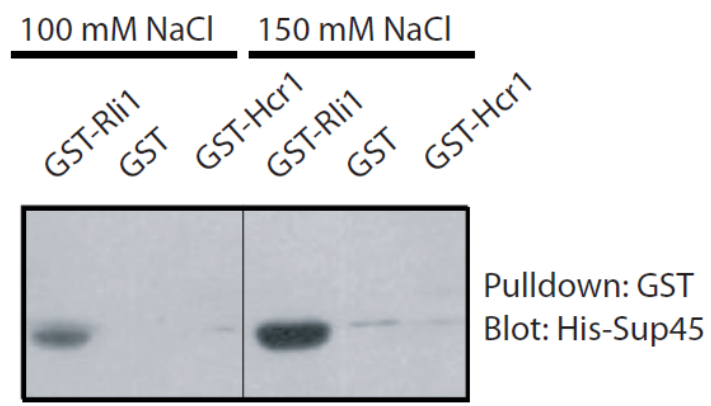

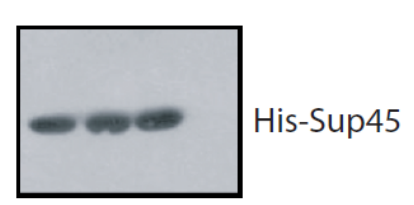

Figure 4. 2. Identification of the Rli1 protein interaction domain for Hcr1 and Sup45. (A) Yeast-twohybrid assay using either full-length or the indicated truncations of Rli1 as bait and either Hcr1 or Sup45 as prey. The domain structure of Rli1 is indicated and positive interactions are indicated as (+), no interaction as (-). (B) GST-pulldown assays with His-tagged Hcr1 performed under the indicated salt concentrations by using full-length Rli1 (GST-Rli1) or GST and GST-PNC1 as negative controls. The eluate fractions (top) and the input (bottom) are shown. (C) GST-pulldown assays with His-tagged Sup45 and GST-Rli1, GST alone or GST-Hcr1 were performed as described in (B). 


\section{Rli1 is required for proper stop codon recognition}

Physical and genetic interactions between Rli1 and the termination factors eRF1 and eRF3 indicate a novel function of Rlil in translation termination. To test this directly, we investigated if the downregulation of Rlil would lead to defects in the recognition of a stop codon. For this purpose we used an established dual reporter assay that detects read through efficiency (Bhattacharya et al. 2000). The assay is based on compared expression of $\beta$-galactosidase and luciferase open reading frames, separated by a stop codon (Figure 4.4.A). As a control we also used a reporter with a stem loop. This assay allowed us to compare the frequency of translational readthrough in different strain backgrounds, such as the rat8-2 strain, which has known mRNA export defects (Cole and Scarcelli 2006). All measured luciferase activity was therefore calculated in relation to the B-galactosidase activity (Figure 4.4.B-C and supplementary Figure S4.4.B). Similar to the rat8-2 mutant strain, the downregulation of $R L I 1$ results in a $\sim 50 \%$ reduced translation rate (supplementary Figure S4.3.A), which in case of $R L I 1$ is due to defects in translation initiation (Dong et al. 2004), however, the dual reporter construct allowed us to analyze the true defects in translation termination by calculating the ratio of luciferase activity in regard to the $\beta$-galactosidase activity (an example is shown in supplementary Figure S4.3.B). In agreement with previous results, we found a basal read through activity of $\sim 15$ to $\sim 17 \%$ in wildtype cells (Bhattacharya et al. 2000). Although the repression of RLII did not influence readthrough activities in the presence of the stem loop, Rli1 was clearly required for efficient recognition of the termination codon, similar to the other termination factors eRF1, eRF3 and Dbp5 (Figure 4.4.B).

In addition, we found that the defects of sup 45-2 in termination codon recognition were partially suppressed by overexpression of $R L I 1$ using the strong TDH3-promoter. Yeast cells containing the reporter construct and either an empty vector $(p)$ or plasmids encoding wildtype RLII (TDH3:RLI1) or a mutant defective in the Fe/S cluster (TDH3:rli1 (C28S)) were grown to the logarithmic growth phase, before the termination readthrough activity was determined. As shown in Figure 4.4.C, overexpression of wildtype RLI1, but not the Fe/S-cluster mutant was able to partially suppress the termination readthrough defects of sup 45-2. The functionality of the TDH3:RLI1 plasmid is shown in supplementary Figure S4.2. 
A
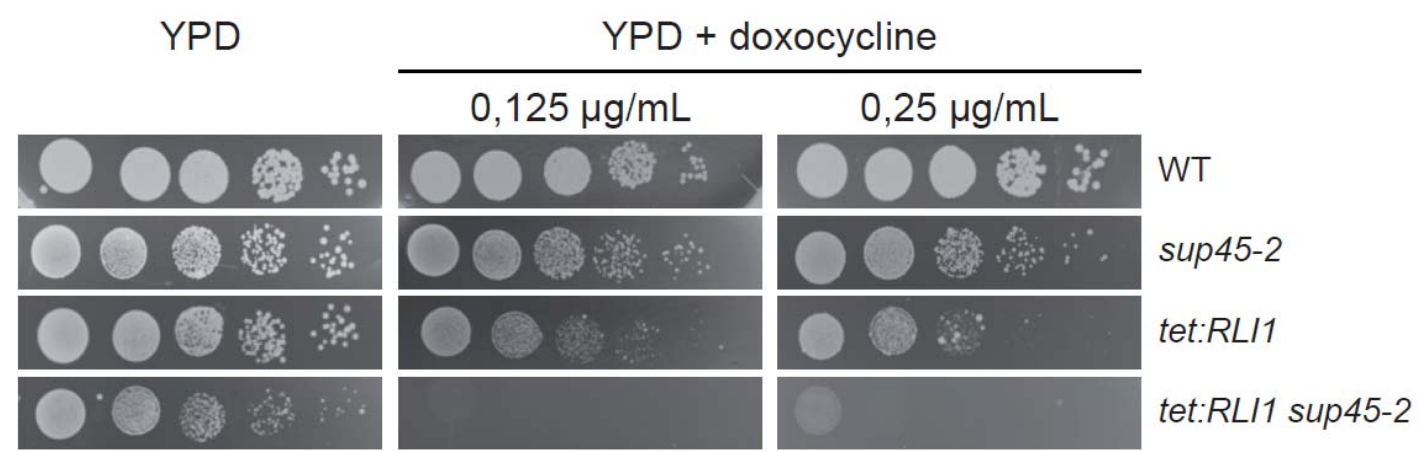

B
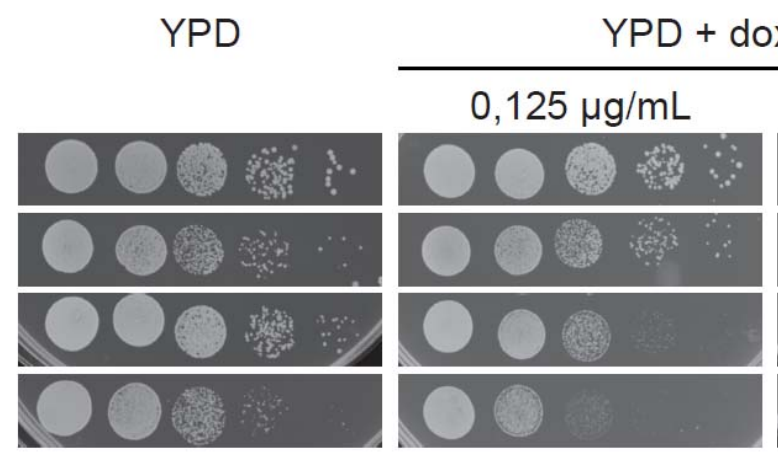

oxocycline

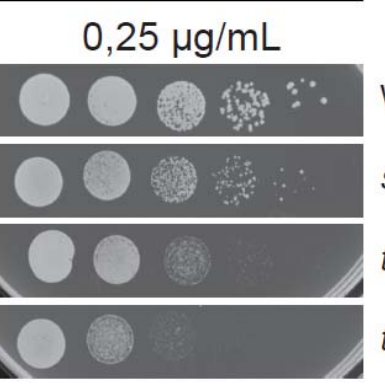

\section{WT}

sup35-21

tet:RLI1

tet:RLI1 sup35-21

Figure 4. 3 Genetic interactions between RLI1 and both translation termination factors eRF1 and eRF3. (A) Serial dilutions of wildtype, sup45-2, tet:RLI1 and the double mutant tet:RLI1 sup45-2 were spotted onto full medium plates (YPD) and on plates containing the indicated concentrations of doxocycline, that down-regulates the RLI1 expression are shown after incubation at $25^{\circ} \mathrm{C}$ for three days. (B) Serial dilutions of wildtype, sup35-21, tet:RLI1 and the double mutant tet:RLI1 sup35-21 are shown on YPD- and doxocycline containing-plates after incubation at $25^{\circ} \mathrm{C}$ for three days.

Overexpression of RLII in wildtype cells is toxic and leads to translation initiation defects (Dong et al. 2004). The interaction domain of Rli1 with both, Hcr1 and Sup45, was determined to the $2^{\text {nd }} \mathrm{ABC}$ domain of the protein. Therefore one would expect that overexpression of this domain in wildtype cells might disturb those interactions and inhibit cellular growth. Indeed, expression of the $2^{\text {nd }} \mathrm{ABC}$ domain from the strong GAL1 promoter leads to inhibition of growth (supplementary Figure S4.4.A), however, not to termination read through defects (supplementary Figure S4.4.B). This could have several reasons: Expression of the ABC domain might result in an unnatural folding of this domain or it might remain associated with Hcr1 in the initiation complex, or associate with other proteins to which the full length protein has no contact. 
A

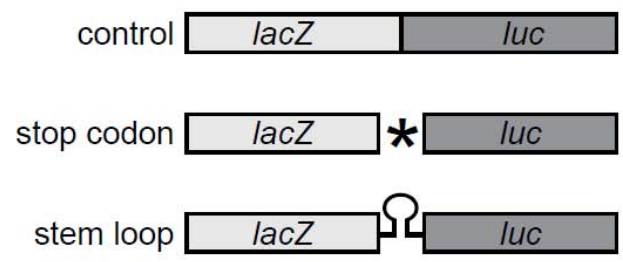

B

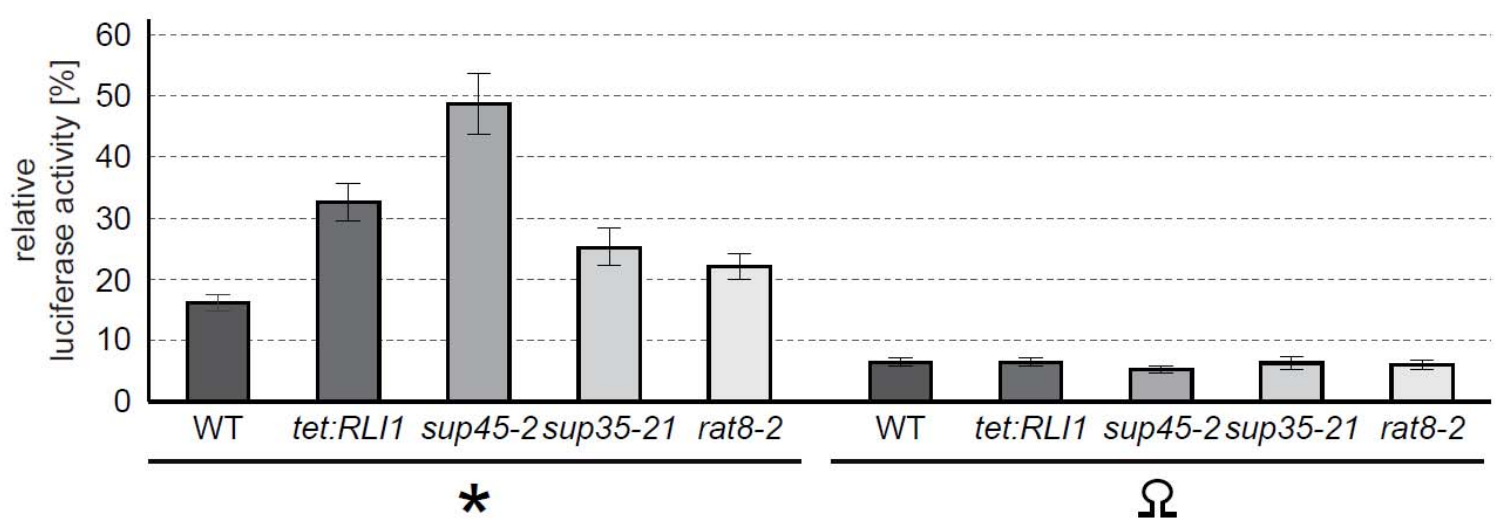

C

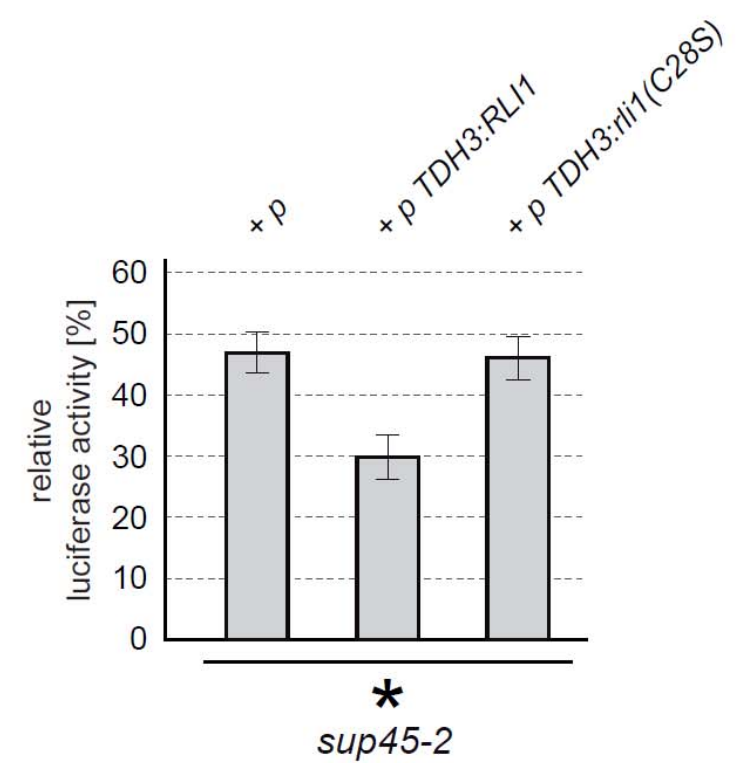

Figure 4. 4 Rli1 is required for efficient stop codon recognition. (A) Scheme of used the reporter constructs for determination of the readthrough activity. (B) Readthrough activities are shown for wildtype, a strain that downregulates the RLI1 expression (tet:RLI1), sup45-2, sup35-21 and rat8-2, encoding the defective DEAD-box RNA-helicase Dbp5. All strains carrying either of the reporter constructs were grown to log phase and shifted to $37^{\circ} \mathrm{C}$ for $15 \mathrm{~min}$ before cell lysis. ß-galactosidase and luciferase activities were measured and their ratios were used to calculate the relative molar luciferase expression. (C) Suppression of the termination readthrough defects of sup45-2 by increased Rli1 level (TDH3:RLI1), but not an Fe/S cluster mutant (TDH3:rli1(C28S) is shown. All strains carrying the reporter construct and the indicated plasmids were treated as described in (B). The results of at least 10 independent experiments are shown. 
Taken together our results indicate that Rli1 is required for translation termination as the protein interacts with both termination factors eRF1 and eRF3 in vivo (Figure 4.1). Rli1 may regulate the termination process via eRF1, since a strong genetic interaction was specifically detected with a mutant of eRF1 (sup45-2) (Figure 4.3). Consistently, we find that the physical interaction between Rli1 and eRF1 is direct, since no additional factor is required for the interaction in vitro (Figure 4.2.C). Moreover, while the repression of the RLII-expression leads to defects in the recognition of a stop codon, its overexpression partially suppresses the readthrough defects of an eRF1 mutant (sup45-2) (Figure 4.4.B). Interestingly, although we show that the Fe/S cluster is not required for the interaction of Rli1 with eRF1 or its other interacting partner Hcr1 (Figure 4.2 ), it is required for its activity in translation termination, as a Fe/S cluster mutant of Rli1 is not able to suppress the readthrough defects of sup45-2 (Figure 4.4C). We rather mapped the protein interaction domain of Rlil to its second C-terminal ATPase domain (Figure 4.2). Interestingly Rlil requires the $2^{\text {nd }} \mathrm{ABC}$ domain for its interaction with the termination factor eRF1 and also with the initiation factor Hcr1, as a component of the translation initiation factor eIF3. Hcr1 has recently been shown to play a role ribosomal subunit dissociation and recycling together with eRF1 (Pisarev et al. 2007). Thus, it is tempting to speculate that Rlil might also be involved in ribosome recycling, however since the disassembly of post-termination complexes was successfully be performed with purified eIF3/Hcr1 and eRF1 (Pisarev et al. 2007), a potential role of Rli1 is limited. Future experiments are needed in order to investigate potential functions of Rli1 beyond translation termination.

\subsection{Materials and Methods}

\section{Strains and plasmids}

All yeast strains used in this study are listed in the supplementary material in table S1, plasmids in table S2. In order to analyze several proteins in the same Saccharomyces cerevisiae strain, we used wildtype cells with genomically tagged genes and plasmids encoding functionally tagged versions of the analyzed proteins. Strains were cultivated in standard media at $25^{\circ} \mathrm{C}$. Details on strain constructions and cloning strategies can be found in the supplemental data. All growth tests are shown in 10 -fold serial dilution starting with approximately $10^{5}$ cells per drop.

\section{Yeast-two-hybrid library screen}

Full-length $R L I 1$ was fused to the DNA binding domain of the transcriptional activator GAL4 and used as bait for a two-hybrid screen of a yeast cDNA library fused to the GAL4 activation 
domain. A detailed description of the procedure is described in supplementary material.

\section{Co-Immunoprecipitations}

The experimental procedure was essentially performed as in Gross et al., 2007. Coimmunoprecipitations were performed using Protein G sepharose beads (Amersham Biosciences) conjugated to HA-specific antibodies or $\operatorname{IgG}$ sepharose beads (Amersham Biosciences) in presence or absence of $200 \mu \mathrm{g} / \mathrm{ml}$ RNase. Purification of the TAP-tagged proteins was performed under similar conditions. A detailed description of the procedure is described in supplementary material.

\section{GST-pulldown}

Rosetta II (DE3) cells were transformed with pGEX6P3-Rli1 and either pET30a-Sup45 or pET28b-Hcr1. After cell lysis and removal of cell debris, the supernatant was incubated with GSH-sepharose beads. After several washing steps, the beads were analyzed on SDS page followed by western blot and immunostaining. For a comprehensive protocol, see supplementary material.

\section{Dual-reporter ß-galactosidase luciferase assay}

The reporter assays were essentially done as described (Bidou et al. 2000). Different transformants of strains carrying the dual-reporter vector and, if indicated plasmids that over expressing RLI1-HA or rlil(C28S)-HA, were grown to $\mathrm{OD}_{600}=0.6-0.8$ and lysed after a $30 \mathrm{~min}$ temperature shift to $37^{\circ} \mathrm{C}$. B-galactosidase and luciferase activities were assayed from the same cell culture, as described previously (Bidou et al. 2000). Cells were broken using acid-washed glass beads and vigorous vortexing for $20 \mathrm{sec}$ and $4.5^{\circ} \mathrm{m} / \mathrm{s}$ using FastPrep ${ }^{\circledR}-24$ (MP Biomedicals). The recoding efficiency, expressed in percentage, was determined by dividing the luciferase/ß-galactosidase ratios obtained from each test conduct by the same ratio obtained with the appropriate in-frame control. See also (Bidou et al. 2000).

\subsection{Acknowledgement}

We thank D. M. Bedwell, R. Lill and U. Mühlenhoff, for strains and/or plasmids and/or antibodies and B. Boll and L. Schreiber for plasmids and experimental assistance. 


\subsection{Supplementary Information}

\section{Construction of GST- and pentaHis- fusion proteins and the GAL:RLI1 vectors}

To construct GST-fusion proteins of RLI1, oligonucleotides corresponding to the 5'-, and 3'-ends or internal sequences were used for PCR with yeast genomic DNA as template and digested with appropriate restriction enzymes corresponding to the terminal sequences (BamH1 or SmaI at the 5'-end and XhoI at the 3'-end) and subcloned into p GEX6P-3. To insert a polyhistidine tag (Histag) into the ORF of SUP45 or HCR1, oligonucleotides corresponding to the 5'-, and 3'-ends were amplified from yeast genomic DNA by PCR, introducing terminal BamHI and XhoI sites, respectively. The BamHI-XhoI fragments of the amplified DNA were subcloned into pET28b (HCR1) or pET30a (SUP45), respectively. Primer sequences are listed in table S3.

To generate GAL:RLI1 constructs, DNA fragments containing either full-length RLI1 or the Cterminal ABC domain were PCR amplified using the primers listed in TABLE S3 and cloned into p426GAL1 using SmaI and XhoI sites.

\section{Yeast-two-hybrid library screen}

A DNA fragment containing either the complete ORF of RLI1 or its different truncations were PCR-amplified using the oligonucleotides listed in table S3 and cloned into pGBKT7 using the Clontech Matchmaker system (Fields and Sternglanz 1994; James et al. 1996; James 2001). This construct was used as bait for a two-hybrid screen of a yeast cDNA library fused to the GAL4 activation domain in pGAD GH (kindly provided by Prof. D. Gallwitz (Max Planck Institute for Biophysical Chemistry Göttingen)). Purified DNA from the libraries was transformed into yeast strain AH109 carrying the bait plasmid pGBKT7-RLI. Transformants were plated onto the triple dropout plates (SD/-trp/-leu/-his). Transformants that grew on the triple dropout plates were rescreened under more stringent conditions on $\mathrm{SD} /$-trp/-leu/-ade/-his dropout plates to test for $A D E 2$ expression and on $\mathrm{X}$-gal- filter assays to test for lacZ expression. Plasmids were isolated from yeast transformants that were his + , ade+, and blue on X-gal and the inserts adjacent to the GAL4 domain were sequenced.

\section{Co-immunoprecipitation and protein purification}

Cells were grown at $25^{\circ} \mathrm{C}$ to a density of $\mathrm{OD}_{600}$ 0.5-0.8 in the appropriate $\mathrm{SD}$ medium. If indicated a temperature shift to $37^{\circ} \mathrm{C}$ was done before $\mathrm{OD}_{600}$ of the cells were collected, washed once in $\mathrm{ddH}_{2} \mathrm{O}$ and resuspended in $600 \mu \mathrm{l}$ of PBSMKT-buffer $(137 \mathrm{mM} \mathrm{NaCl}, 5.7 \mathrm{mM} \mathrm{KCl}$, 
$10 \mathrm{mM} \mathrm{Na} 2 \mathrm{HPO}_{4}, 2 \mathrm{mM} \mathrm{KH} \mathrm{PO}_{4}, 2.5 \mathrm{mM} \mathrm{MgCl}_{2}, 0.05-0.5 \%$ (v/v) Triton X-100). Yeast cells were transfered to microcentrifuge tubes, one cell volume of glass beads and protease inhibitor cocktail (Sigma-Aldrich (Nr. P8215) and Roche Applied Science (Nr. 11873580001)) were added, and the cells were lysed by vigorous vortexing for $20 \mathrm{sec}$ and $4.5^{\circ} \mathrm{m} / \mathrm{s}$ using FastPrep ${ }^{\circledR}$ 24 (MP Biomedicals). After spinning for $5 \mathrm{~min}$ at $16.000 \mathrm{x} \mathrm{g}$, the clarified supernatants were split into two equal portions (+/- addition of RNase A) and incubated for $2-4 \mathrm{~h}$ at $4^{\circ} \mathrm{C}$ with gentle agitation either with $10 \mu 1$ of Protein G sepharose beads (Amersham Biosciences) or with $10 \mu 1$ IgG sepharose beads (Amersham Biosciences), conjugated to monoclonal c-myc (9E10)- or polyclonal GFP-specific antibodies. In the RNase containing samples the enzyme was added to the PBSMKT-buffer to a final concentration of $200 \mu \mathrm{g} / \mathrm{ml}$. The beads were washed five times with $1 \mathrm{ml}$ PBSMKT-buffer by repeated low speed centrifugation (600 x g). Samples of the lysates, the final washes and the eluates were mixed with $2 \times$ SDS sample loading buffer and separated on $10-15 \%(\mathrm{v} / \mathrm{v})$ SDS-PAGEs.

\section{GST-pulldowns}

Rosetta II (DE3) cells were transformed with pGEX6P3-Rli1.and either pET30a-Sup45 or pET28b-Hcr1. pGEX6P-3 alone or GST-PNC1 (pGEXTT) co-expressend with either HisSup45p or His-Hcr1 served as negative controls. Overexpression was induced by $0.2 \mathrm{mM}$ IPTG. Cells were grown at $16^{\circ} \mathrm{C}$ for $20 \mathrm{~h}$, harvested and resuspended $(50 \mathrm{mM}$ Tris- $\mathrm{HCl}, \mathrm{pH} 7.5$, $200 \mathrm{mM} \mathrm{NaCl}, 2 \mathrm{mM}$ 2-mercaptoethanol, 5\% (v/v) glycerol, $0.2 \mathrm{mM}$ PMSF, protease inhibitor mix). After cell lysis and centrifugation steps, the supernatant was diluted 1:1 with $50 \mathrm{mM}$ Tris$\mathrm{HCl}$, pH 7.5, 2 mM 2-mercaptoethanol, 5\% (v/v) glycerol, $0.2 \mathrm{mM} \mathrm{PMSF,} 2 \mathrm{mM} \mathrm{MgCl} 2,2 \mathrm{mM}$ ATP and incubated with $100 \mu \mathrm{l}$ of GSH-sepharose beads for $2 \mathrm{~h}$ at $4^{\circ} \mathrm{C}$. After several washing steps (50 mM Tris-HCl, pH 7.5, $100 \mathrm{mM} \mathrm{NaCl,} 2$ mM 2-mercaptoethanol, 5\% (v/v) glycerol, $0.2 \mathrm{mM}$ PMSF), beads were analysed on SDS page and by immunostaining. For immunostaining, a penta-His antibody (Quiagen) was employed as first antibody, followed by an alkaline-phophatase conjugated secondary antibody (Dianova) and visualization of the Histagged protein by alkaline phosphatase assay. 
4. Rlil functions in translation termination

\begin{tabular}{|c|c|c|c|}
\hline Systematic name & $\begin{array}{l}\text { Standar } \\
\text { d name }\end{array}$ & Description & $\begin{array}{c}\text { Number of } \\
\text { independent } \\
\text { clones }\end{array}$ \\
\hline YLR192C & HCR1 & rRNA processing/translation initiation & 32 \\
\hline YBL092W & RPL32 & protein component of the $60 \mathrm{~S}$ ribosomal subunit & $2^{*}$ \\
\hline YBR143C & SUP45 & $\begin{array}{l}\text { polypeptide release factor eRF1 involved in translation } \\
\text { termination }\end{array}$ & 1 \\
\hline YHL015W & RPS 20 & protein component of the $40 \mathrm{~S}$ ribosomal subunit & $1^{*}$ \\
\hline YMR143W & RPS16 & protein component of the $40 \mathrm{~S}$ ribosomal subunit & $1^{*}$ \\
\hline YDL031W & DBP10 & $\begin{array}{l}\text { putative ATP dependent helicase of the DEAD box protein } \\
\text { family; involved in } 60 \mathrm{~S} \text { ribosome biogenesis }\end{array}$ & 1 \\
\hline YLR043C & TRX1 & protects cells against both oxidative and reductive stress & 1 \\
\hline YOL016C & $\mathrm{CMK} 2$ & calmodulin-dependent protein kinase & 1 \\
\hline YLR044C & PDC1 & pyruvate decarboxylase; glucose fermentation & 1 \\
\hline YOR047C & STD1 & $\begin{array}{l}\text { protein involved in control of glucose-regulated gene } \\
\text { expression }\end{array}$ & 1 \\
\hline YGR192C & TDH3 & $\begin{array}{l}\text { glycerinealdehyde- } 3 \text { phosphate dehydrogenase; } \\
\text { glycolysis }\end{array}$ & 1 \\
\hline YOR383C & FIT3 & mannoprotein & 1 \\
\hline YOR202W & HIS 3 & histidine biosynthesis & 1 \\
\hline YBL036C & & alanine racemase activity & 1 \\
\hline YDL097C & RPN6 & $\begin{array}{l}\text { essential, non-ATPase regulatory subunit of the } 26 \mathrm{~S} \\
\text { proteasome }\end{array}$ & $1^{*}$ \\
\hline YBR085C & & function unknown & 1 \\
\hline undefined ORF & & & 8 \\
\hline
\end{tabular}

Figure S4. 1. Summary of the results from the yeast-two-hybrid screen. Yeast-two-hybrid identified 55 proteins that gave strong yeast-two-hybrid interaction signals with the three reporter genes, ADE2, HIS3, and lacz. * denotes known false-positives in yeast-two-hybrid screens (Hengen, 1997). 


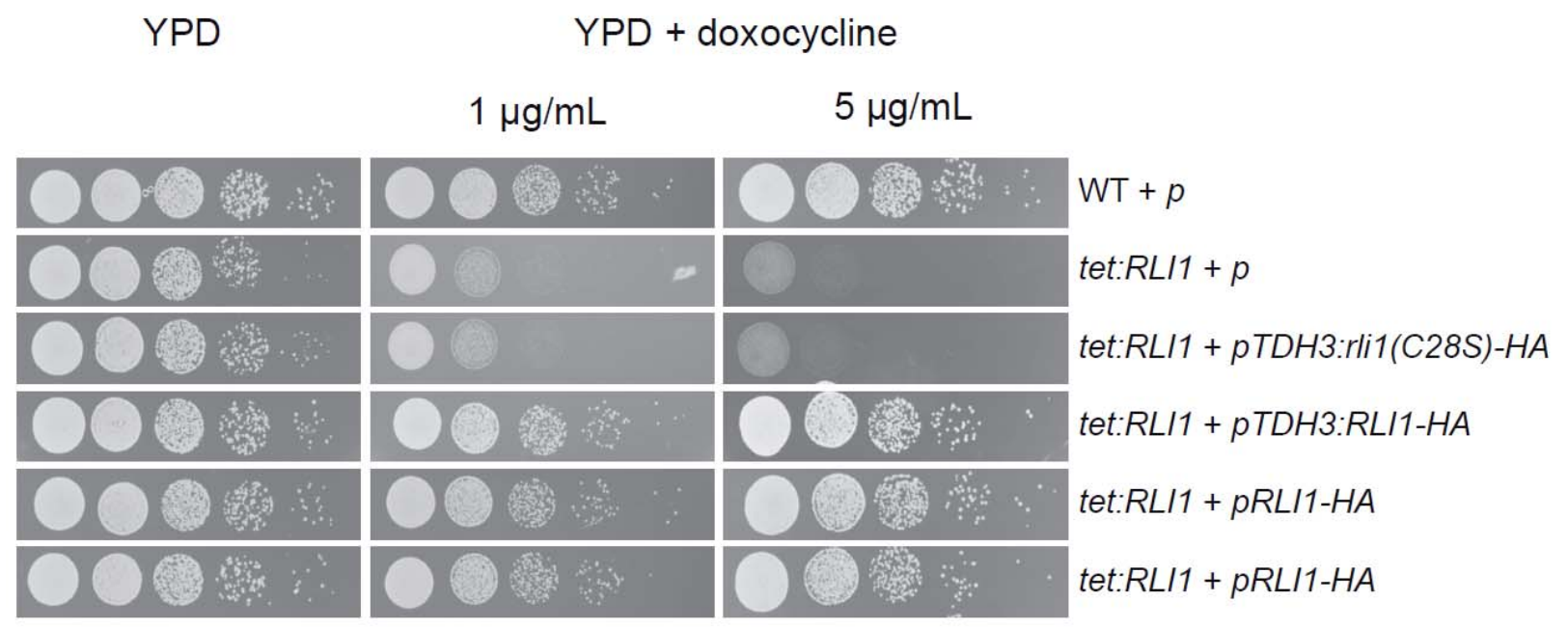

Figure S4. 2. Down-regulation of RLI1 is lethal, but can be suppressed by a functional copy of HAtagged RLI1. Serial dilutions of the indicated strains and plasmids are shown after growth at $25^{\circ} \mathrm{C}$ for three days on either full media (YPD) or YPD plates containing the indicated concentrations of doxocycline to down regulate the RLI1 expression. 
A

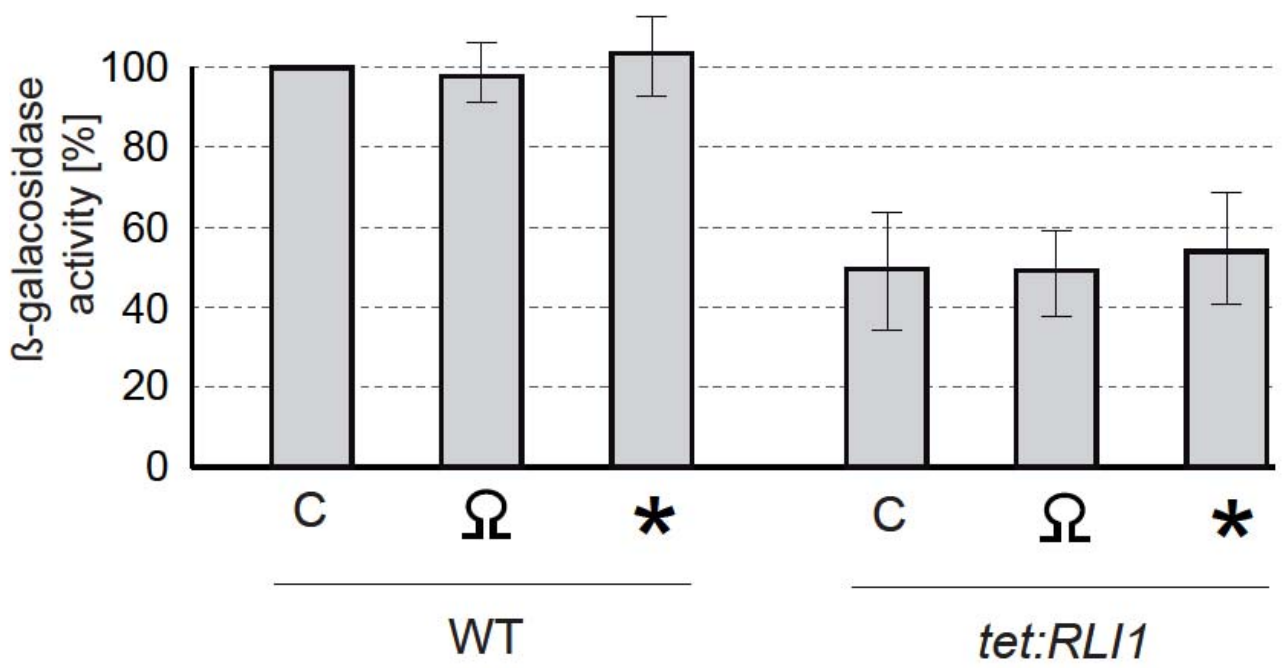

B

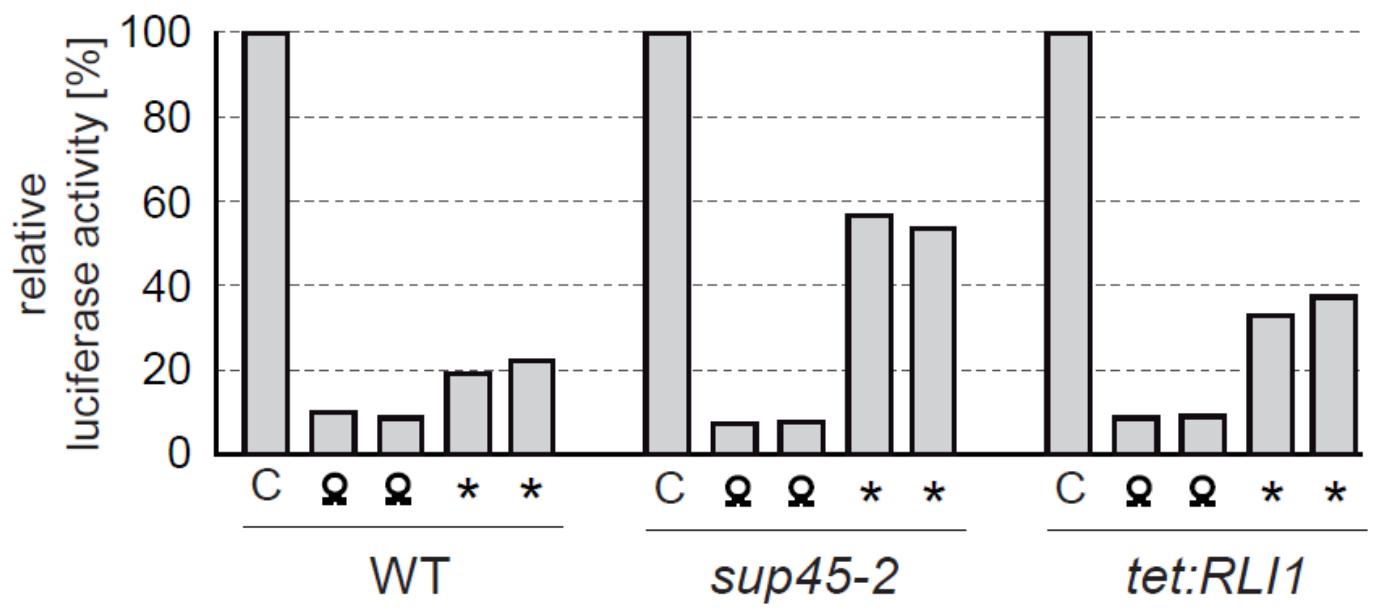

Figure S4. 3. Down-regulation of RLI1 leads to defects in translation and to increased read through activity. (A) Comparison of the ß-galatosidase activities in wildtype and downregulated RLI1 cells (tet:RLI1) with three different vectors as depicted in Fig. $4 \mathrm{~A}\left(C=\right.$ control, $\Omega=$ stem loop, ${ }^{*}=$ stop codon). The ß-galatosidase activity of $1 \mathrm{OD}_{600}$ of wildtype cells (carrying the control vector $\mathrm{C}$ ) is set to $100 \%$. (B) Comparison of the luciferase activities of wildtype, sup45-2 and downregulated RLI1 cells (tet:RLI1) from two different examples of the stem loop containing construct $(\Omega)$ and the stop codon vector $\left({ }^{*}\right) \cdot C=$ control. 
A

\begin{tabular}{|c|c|c|}
\hline Glucose & Galactose & \\
\hline O@繁 & 000 & $\mathrm{WT}+p$ \\
\hline $0 O: \because$ & 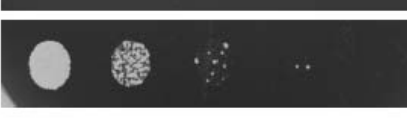 & \\
\hline
\end{tabular}

B

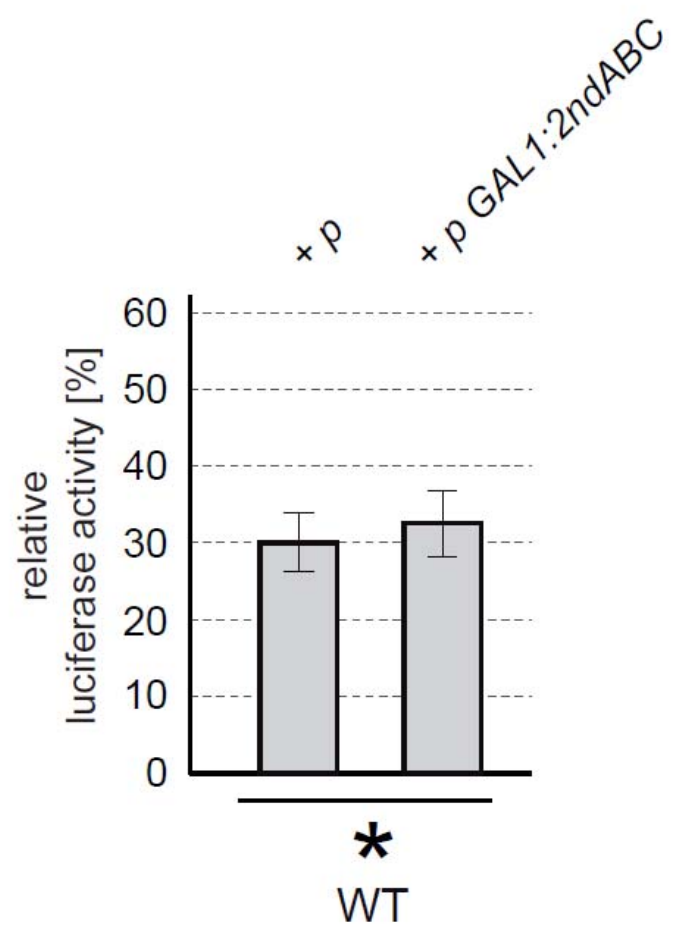

Figure S4. 4. Overexpression of the $2^{\text {nd }} A B C$ domain is toxic, but does not influence the recognition of a stop codon. (A) Overexpression of the $2^{\text {nd }} A B C$ domain is toxic to wildtype cells. Wildtype cells carrying either an empty $2 \mu$ vector $(+p)$ or a $2 \mu$ plasmid encoding the $2^{\text {nd }} A B C$ domain of RLI1, driven from the strong GAL1 promoter were spotted in serial dilutions onto glucose or galactose containing plates and incubated for 2-3 days at $30^{\circ} \mathrm{C}$. (B) Overexpression of the $2^{\text {nd }} A B C$ domain does not influence proper stop codon recognition. Readthrough activities for wildtype cells are shown that carry either an empty vector $(+p)$ or a plasmid encoding the second ABC domain of RLI1, driven from the strong GAL1 promoter. (Basal readthrough activity in galactose containing medium is approximately $30 \%$ ). 
Table S4.1. Yeast strains

\begin{tabular}{|c|c|c|c|}
\hline Number & Name & Genotype & Source \\
\hline HKY35 & FY86 & MATa ura3-52 leu2 21 trp $1 \Delta 63$ & Winston et al., 1995 \\
\hline HKY36 & FY23 & MATa ura3-52 leu2 1 l his $3 \Delta 200$ & Winston et al., 1995 \\
\hline HKY130 & CSY550 & MATa ura3-52 leu $2 \Delta 1$ trp $1 \Delta 63$ rat8-2 & Snay-Hodge et al., 1998 \\
\hline HKY446 & MT552/8a & MATa ura3-1 his5 2 ade2-1 can1-100 sup 45-2 & Stansfield et al., 1997 \\
\hline HKY473 & & 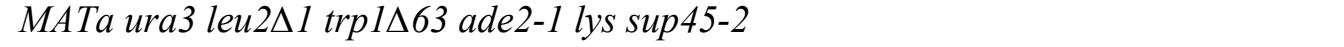 & Gross et al., 2007 \\
\hline HKY486 & SC0117 & MATa ade2 arg4 leu2-3,112 trp1-289 ura3-52 SUP45:TAP:k.l.URA3 & Euroscarf \\
\hline HKY487 & SC0134 & MATa ade2 arg4 leu2-3,112 trp1-289 ura3-52 SUP35:TAP:k.l.URA3 & Euroscarf \\
\hline HKY552 & 21-33G-D373 & $\begin{array}{l}\text { MATa pheA10; ade2-144,717 his7-1(UAA); lys9-A21(UAA); trp1-289(UAG) ura3-52 } \\
\text { leu2-3,112 sup35-21 }\end{array}$ & Cosson et al., 2002 \\
\hline HKY940 & & MATa ade2-1 his- $\Delta 11,15$ leu2-3,112 ura3-1 can1-100 kanMX:TetO7:rli1 & Kispal et al., 2005 \\
\hline HKY955 & & 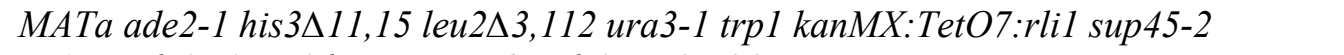 & this study \\
\hline HKY999 & & MATa ade2A1 trp1 kanMX:TetO7:rli1 sup35-21 & this study \\
\hline
\end{tabular}


Table S4.2. Plasmids

\begin{tabular}{|c|c|c|c|}
\hline Number & Name & Features & Source \\
\hline pHK86 & pRS314 & CEN TRP1 & Christianson et al., 1992 \\
\hline pHK88 & pRS316 & CEN URA3 & Christianson et al., 1992 \\
\hline pHK102 & pRS424 & $2 \mu T R P 1$ & Christianson et al., 1992 \\
\hline pHK104 & pRS426 & $2 \mu U R A 3$ & Christianson et al., 1992 \\
\hline pHK606 & pAC 1798 & CEN LEU2 lacZ-(HIV-1 stemloop)-luc & Stahl et al., 1995 \\
\hline pHK607 & pACTMV & CEN LEU2 lacZ-(UAG)-luc & Stahl et al., 1995 \\
\hline pHK608 & pACTQ & CEN LEU2 lacZ-luc & Stahl et al., 1995 \\
\hline pHK886 & & CEN URA3 RLI1-HA & Kispal et al., 2005 \\
\hline pHK 888 & & CEN URA3 TDH3:RLI1-HA & Kispal et al., 2005 \\
\hline pHK891 & & CEN URA3 TDH3:rlil(C28S)-HA & Kispal et al., 2005 \\
\hline pHK904 & & $2 \mu U R A 3$ GAL1:RLI1- $2^{\text {nd }} A B C$ & this study \\
\hline pHK907 & p426GAL1 & $2 \mu U R A 3 G A L 1$ & Mumberg et al., 1994 \\
\hline
\end{tabular}


Table S4.3. Oligonucleotides

\begin{tabular}{|c|c|c|}
\hline Construct & Restriction site & Oligonucleotide sequence (5' - 3') forward / reverse \\
\hline $\begin{array}{l}\text { pGADT7_RLI1/ } \\
\text { pGBKT7_RLI1 }\end{array}$ & NdeI, XhoI & $\begin{array}{l}\text { ACGACAGACC } \underline{\text { CATATGAGTGATAAAAACAGTCG/ }} \\
\text { CATACTCGAGTTAAATACCGGTGTTATCCAAGAAA }\end{array}$ \\
\hline $\begin{array}{l}\text { pGADT7_SUP45 / } \\
\text { pGBKT7_SUP45 }\end{array}$ & NdeI, XhoI & $\begin{array}{l}\text { GGAATT } \underline{\text { CATATGGATAACGAGGTTGAAAAAAATAT/ }} \\
\text { ACCG } \underline{\text { TCGAGAATGAAATCATAGTCGGATCCTT }}\end{array}$ \\
\hline pGADT7_DBP10 & NdeI, XhoI & $\begin{array}{l}\text { GGAATTCATATGGCAGGCGTGCAGAAA/ } \\
\text { ACCGCTCGAGCTAAAATTTACGCTTTTTGGAAGG }\end{array}$ \\
\hline $\begin{array}{l}\text { pME2795_RLI1-2 }{ }^{\text {nd }} A B C \\
\text { domain (pHK904) }\end{array}$ & SmaI, XhoI & $\begin{array}{l}\text { TTCCCCGGGAATGACTGAAGCTTTACAATTTAGAATAGC/ } \\
\text { CATACTCGAGTTAAATACCGGTGTTATCCAAGAAA }\end{array}$ \\
\hline $\begin{array}{l}\text { pGBKT7_RLI1-2 }{ }^{\text {nd }} A B C \\
\text { domain / } \\
\text { pGEX6P-1_RLI-2 }{ }^{\text {nd }} A B C \\
\text { domain }\end{array}$ & NdeI, XhoI & $\begin{array}{l}\text { GGAATTCATATGACTGAAGCTTTACAATTTAGAATAGC/ } \\
\text { CATACTCGAGTTAAATACCGGTGTTATCCAAGAAA }\end{array}$ \\
\hline $\begin{array}{l}\text { pGBKT7_HCR1/ } \\
\text { pET22b_HCR1 }\end{array}$ & NdeI, XhoI & $\begin{array}{l}\text { GGGAATTCCATATGTCTTGGGACGACGAAGCTA/ } \\
\text { GCC } \underline{\text { CTCGAGTTACATAAAGTCGTCATCACCAAGTTC }}\end{array}$ \\
\hline pET30a_SUP45 & BamHI, XhoI & $\begin{array}{l}\text { ACGCGGATCCATGGATAACGAGGTTGAAAAAAATAT/ } \\
\text { ACCG } \underline{\text { TCGAGAATGAAATCATAGTCGGATCCTT }}\end{array}$ \\
\hline
\end{tabular}




\section{Chapter $5 \bullet$ Discussion}

Eukaryotic translation initiation is an intimately regulated process involving the orchestrated function of the ribosome, mRNA and several initiation factors. This process brings about the accurate deciphering of the authentic start codon, leading to the establishment of the correct reading frame for the protein synthesis. The intricately regulated interaction of the initiation factors is the key to achieve the high fidelity of initiation. A key player of most of the initiation events is the multi-subunit scaffolding eIF3 complex, which on one hand facilitates formation of 43S PIC and on the other hand plays a role in mRNA recruitment and $80 \mathrm{~S}$ ribosome dissociation. The scaffolding nature of eIF3 hinders the crystallization of the whole complex. In addition, having too many interacting partners makes the purification of the contaminant-free eIF3 difficult, hampering in vitro biochemical and structural studies of the complex. To circumvent these problems, a recombinant purification and in vitro reconstitution procedure was established during this thesis work, which allows obtaining highly pure eIF3 complex in large scale.

\subsection{Recombinant purification and in vitro reconstitution of eIF3}

The complex structural organization of eIF3 and the scaffolding role it plays during translation initiation make the native purification of pure eIF3 difficult. One of the major contaminations of eIF3 is eIF5, the GTPase activating protein (GAP) of eIF2. The contamination of eIF3 with the eIF2-GAP makes it unreliable to use eIF3 in the biochemical studies involving the hydrolysis of eIF2-GTP. In attempts to purify eIF3 from its native source according to the published protocols (Acker et al. 2007), additional bands corresponding to eIF5 were co-purified with eIF3 (Figure 2.2). To overcome the problem of the co-purification of unwanted proteins, the coding sequences of all six components of yeast eIF3 were cloned into bacterial expression vectors and purified recombinantly. The eIF3 complex was reconstituted by mixing Tif32/Prt1/Nip1 ternary complex with the binary complex of Tif34/Tif35. Since eIF3 has no enzymatic activity, it is hard to accurately assess the functionality of the complex. However, ribosome binding assay has been suggested as an acid test for the activity of eIF3 (Acker et al. 2007). The reconstituted eIF3 was shown to be active as it could bind to the $40 \mathrm{~S}$ subunit (Figure 2.4). To compare the structural similarity between the recombinant and native eIF3s, both complexes were studied by cryoEM (collaboration with group of Prof. Holger Stark, MPI for Biophysical Chemistry, Goettingen).

Several subunits of eIF3 are subject to post-translational phosphorylation (Smolka et al. 2007). 


\section{Discussion}

However, there has not been any data so far indicating the importance of these modifications for the fundamental functions of yeast eIF3. Although the recombinantly reconstituted eIF3 lacks the post-translational modifications, it still represents a functional unit which is not suffering from the heterogeneities connected with the native purification procedures and hence is suitable for further biochemical studies (Figure 2.4).

\subsection{Insights into the Prt1/Tif34/Tif35 and Tif32/Nip1 sub-complexes of eIF3}

Tif34 and Tif35 are two smaller subunits of eIF3, which bind to the rest of the complex via one of its large subunits, Prt1. Although binary complexes between Tif34 with both Prt1 and Tif35 could be obtained, attempts to reconstitute the binary complex between Tif35 and Prt1 remained unsuccessful, suggesting that Tif34 is probably bridging between the two proteins (Figure 2.3). The fact that Tif34 is predicted to be a $\beta$-propeller makes it an ideal candidate for bridging different proteins together. $\beta$-propeller structures are known to serve as docking sites for other proteins ( $\mathrm{Li}$ and Roberts 2001). Analysis of the thermodynamics of interactions of Tif34 with Prt1 and Tif35 suggested the formation of a tight complex between Tif34 and Tif35 which is connected with lower affinity to the rest of eIF3 complex via Prt1. Later, this instability was accounted for most of the heterogeneity observed during EM analysis (see also below). Controlled proteolytic digestion of Prt1/Tif34/Tif35 sub-complex with thermolysin resulted in a cleavage at the very C-terminus of Prt1, which caused the rest of the protein to migrate independently of Tif34/Tif35 (Figure $2.10 \mathrm{C}$ and D). This result is in accord with the previous reports that the C-terminal domain of Prt1 is responsible for the interaction with Tif34 and Tif35 (Asano et al. 1998). Based on these results, a new model for the Prt1/Tif34/Tif35 sub-complex of eIF3 is proposed in which Tif35 is bound to Prt1 via Tif34 (Figure 5.1).

Proteolytic digestion of the binary complex of Tif32/Nip1, which together form the main body of eIF3, mapped their interaction sites to the C-terminal domain of Nip1 (residue 240 to the end) and the N-terminal domain of Tif32 (residues 1-544; Figure 5.1). Tif32 and Nip1 are the subunits of eIF3 which are known to make critical contacts with other initiation factors in the multi-factor complex (MFC), required for the integrity of MFC and 40S binding (Valásek et al. 2003; Valásek, Nielsen, and Hinnebusch 2002; Asano et al. 2001). C- and N-termini of Tif32 and Nip1 are implicated in these interactions, respectively. Interestingly, sequence-based alignments of Tif32 (eIF3a) and Nip1 (eIF3c) among different eukaryotes show that the highest level of conservation is observed in the $\mathrm{N}$ - and C-termini of Tif32 and Nip1, respectively (Figures 5.2 and 5.3). These domains, being away from the domain responsible for the interaction with eIF2, 


\section{Discussion}

eIF5 and eIF1, are preserved in the limited proteolysis studies and interact with each other. The fact that eIF1-2-5 interacting domains on Tif32 and Nip1 are not preserved during limited proteolysis suggests them to be highly flexible, in line with secondary structure and disorder predictions. The high flexibility might be a prerequisite to search for the interaction partners and fish them out of the pool of other cytosolic proteins, perhaps in a similar way as employed by ribosomal proteins L7 and L12 (Diaconu et al. 2005; Kavran and Steitz 2007). Lower conservation of these domains may point to different modes of interaction with eIFs 1,2 and 5 among different species.

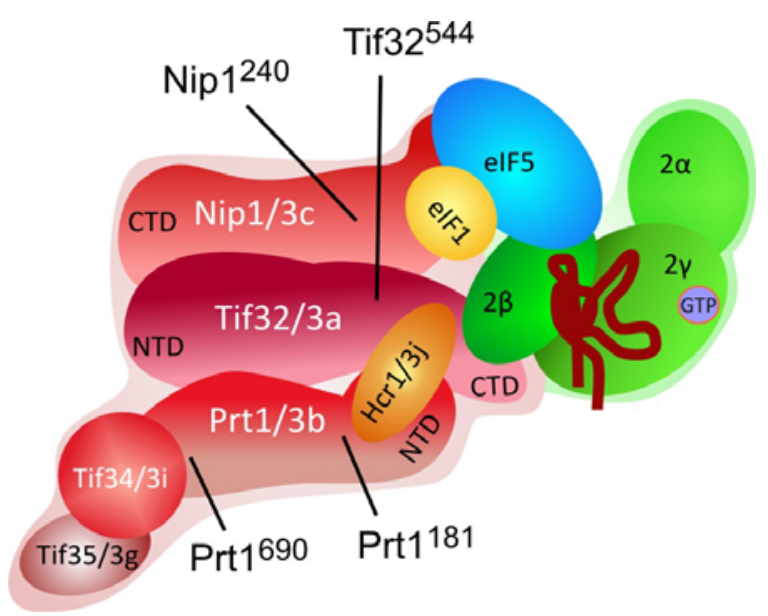

Figure 5.1. Schematic model of yeast MFC. In this mode, Tif35 is repositioned in a way that binds to Prt1 via Tif34 (compared with Figure 1.7). Numbers and black lines represent the boundaries of the proteolytic fragments.

\subsection{Preliminary kinetic insights into the formation of eIF3 and its binding to the 40S subunit using in vitro reconstituted fluorescence-labeled eIF3}

Despite detailed kinetic studies of translation initiation events in prokaryotes, eukaryotic initiation is a challenge owing to its higher complexity. Contrary to three prokaryotic factors (IFs 1-3) there are around ten eukaryotic initiation factors, some of which encompassing more than one subunit, which finely regulate this process. To reduce the complexity, a short unstructured RNA is used which bypasses the requirement for some eIFs. eIF3, as the largest initiation factor, plays a crucial role in different aspects of initiation. However, due to its complexity and problems tied with its purification (such as co-purification of the GAP eIF5), eIF3 has not been subjected to many studies. Having in hand the in vitro reconstituted recombinant eIF3, the kinetics of initiation complex formation could be dissected using fluorescence-labeling of the largest subunit of eIF3, Tif32. 


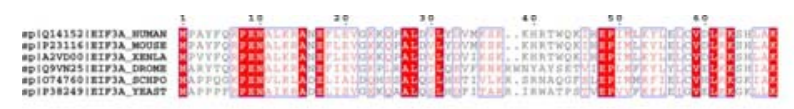

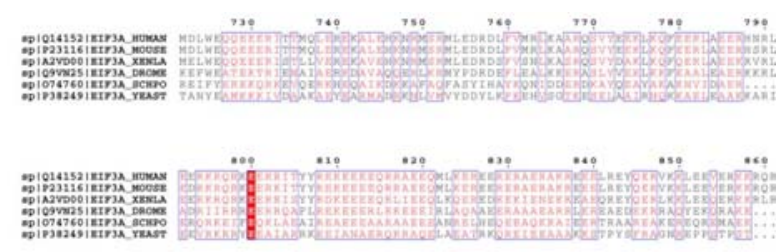

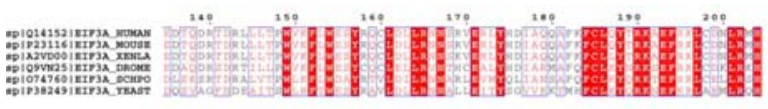
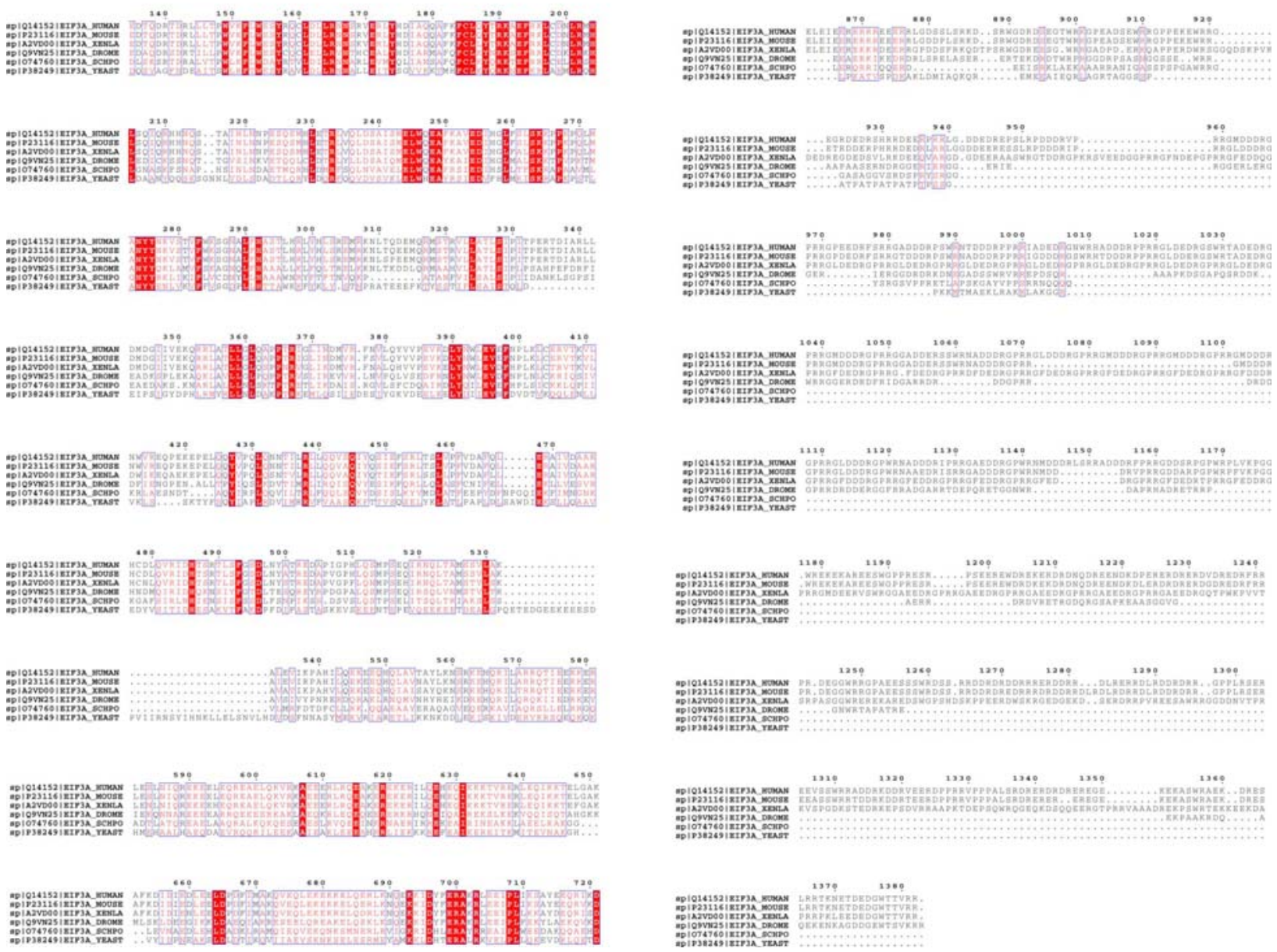

Figure 5.2. Sequence based alignment of elF3a from six different species. The following organisms have been used: Homosapiens sapiens (HUMAN), Mus musculus (MOUSE), Xenopus laevis (XENLA), Drosophila melanogaster (DROME), Schizosaccharomyces cerevisiae (SCHPO) and Saccharomyces cerevisiae (YEAST). Note the conservation of the $\mathrm{N}$-terminal region among different species.

The first kinetic insights into the formation of the eIF3 complex were obtained between two large subunits of eIF3, Nip1 and Tif32 (Figure $2.5 \mathrm{~A}$ ). The resulting curve could be fitted to a double exponential function with a rapid first phase followed by a moderate second phase lasting almost the whole experiment with apparent binding constant of $1.832 \mathrm{~s}^{-1}$. To obtain a more complete picture of the binding, the dissociation constant $\left(K_{d}\right)$ and off rate $\left(k_{o f f}\right)$ have to be measured using ITC and chase experiments, respectively. Stepwise formation of the complex and comparison of the resulting on and off rates will provide the keys to elucidate the mode of complex formation. 


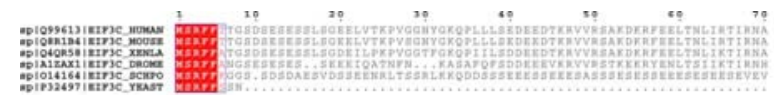

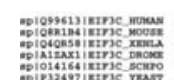
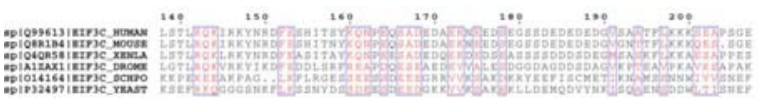

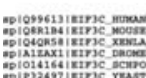
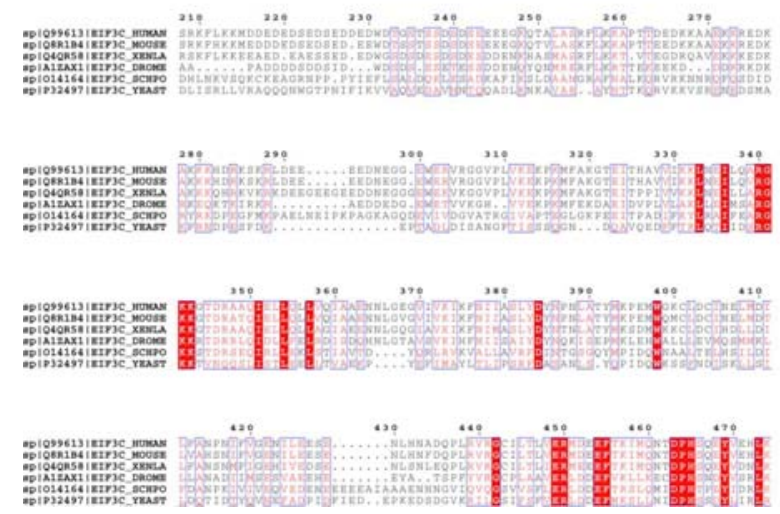
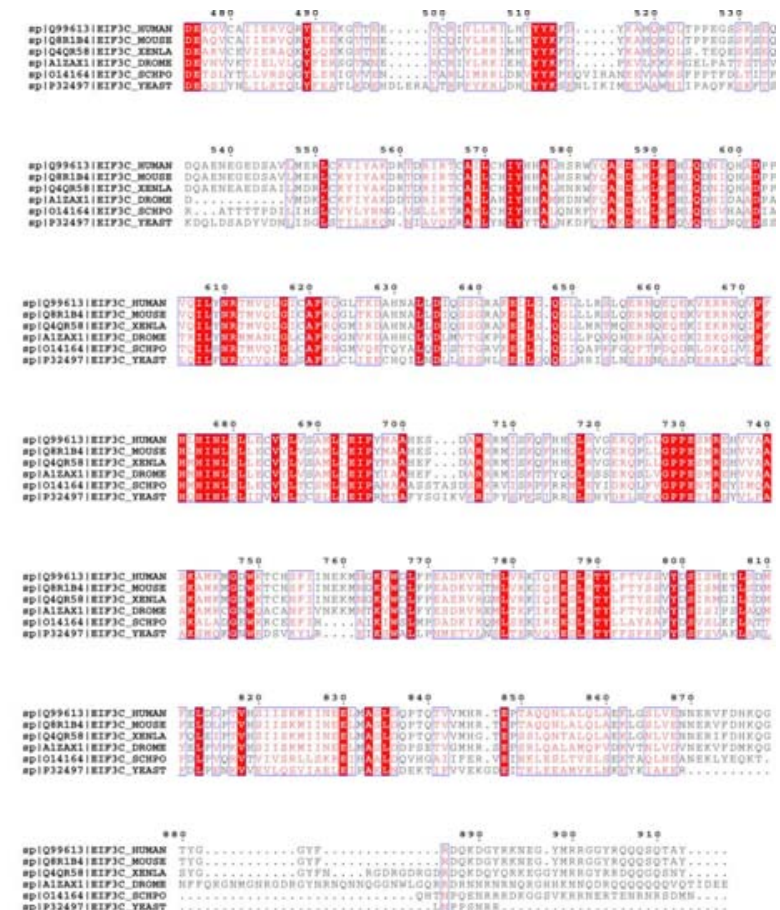

Figure 5.3. Sequence based alignment of elF3c from six different species. The organisms used for alignment are the same as Figure 5.2. Note the conservation of the C-terminal region among different species.

Tif32, Prt1 and Nip1 are shown to form the core of eIF3 required for its association with the ribosome in a manner stimulated by Hcrl (Valásek et al. 2003; Valásek et al. 2001). Formation of this complex with the labeled Tif32 in the absence or presence of $40 \mathrm{~S}$ subunit, followed by a chase of unlabeled Tif32 suggested an effect of the ribosome on the complex (Figure 2.5 B). The observed decrease in the fluorescence in the presence of 40S subunit and not in its absence might indicate structural rearrangements of eIF3 bound to the ribosome compared to free eIF3 which facilitates dissociation and reassociation of Tif32 from the complex. However, using this data we cannot rule out the possibility that in the presence of $40 \mathrm{~S}$ subunit the positioning of the fluorophore is different leading to the observed difference between free and in-complex Tif32.

\subsection{Preliminary EM studies on eIF3}

In order to gain structural insights into the pentameric eIF3 complex either alone or in $40 \mathrm{~S}$ subunit bound state, the most prominent approach is electron microscopy. Human eIF3, a complex of $800 \mathrm{kDa}$, was studied by electron microscopy at low resolution (Siridechadilok et al. 2005). However, as the ribosome-bound complex could not resist the grid-preparation treatments, it was model it on the ribosome. Yeast eIF3 is less than half of its human homolog in size, which makes it a difficult sample for EM. Our reconstituted eIF3 also dissociated into 


\section{Discussion}

several sub-particles in initial studies yielding an inhomogeneous sample (Figure 2.6 A). However, applying the GraFix method (Kastner et al. 2005) helped to stabilize the complex resulting in visually much better samples with more defined particles (Figure 2.6 B). Preliminary 3D reconstruction has obtained a particle with two monomers which are related to each other by a two-fold symmetry (Figure 5.5 A-B).

To structurally compare recombinant and native eIF3s (eIF3 ${ }^{\text {rec }}$ vs. eIF3 ${ }^{\text {nat }}$, respectively), they were analyzed by EM. Initial classification of the 2D projections has yielded class-averages which in many cases resemble a five-lobed particle in the case of eIF3 ${ }^{\text {nat }}$ (Figure 5.4). The longest dimension of these particles is about $200 \AA$, very similar to that of eIF3 ${ }^{\text {rec }}$ (Figure 2.9). Due to the complexity and flexibility of the sample, the advantage of the structural rigidity of the ribosome was exploited. For this, eIF $3^{\text {nat }}-40 \mathrm{~S}$ complex was prepared and used for cryo-EM studies. These images will be used for reconstruction of eIF $3^{\text {nat }}-40 \mathrm{~S}$, which in turn would help to solve the structure of free eIF $3^{\text {nat }}$. The latter structure could be then used to reconstruct eIF $3^{\text {rec }}$ structure and its subcomplexes more reliably. Comparing the structure of eIF $3^{\text {rec }}$ and eIF ${ }^{\text {nat }}-40 \mathrm{~S}$ will provide insight to the binding site of different subunits of eIF3 on the 40S subunit and shed light on eukaryotic translation initiation process.

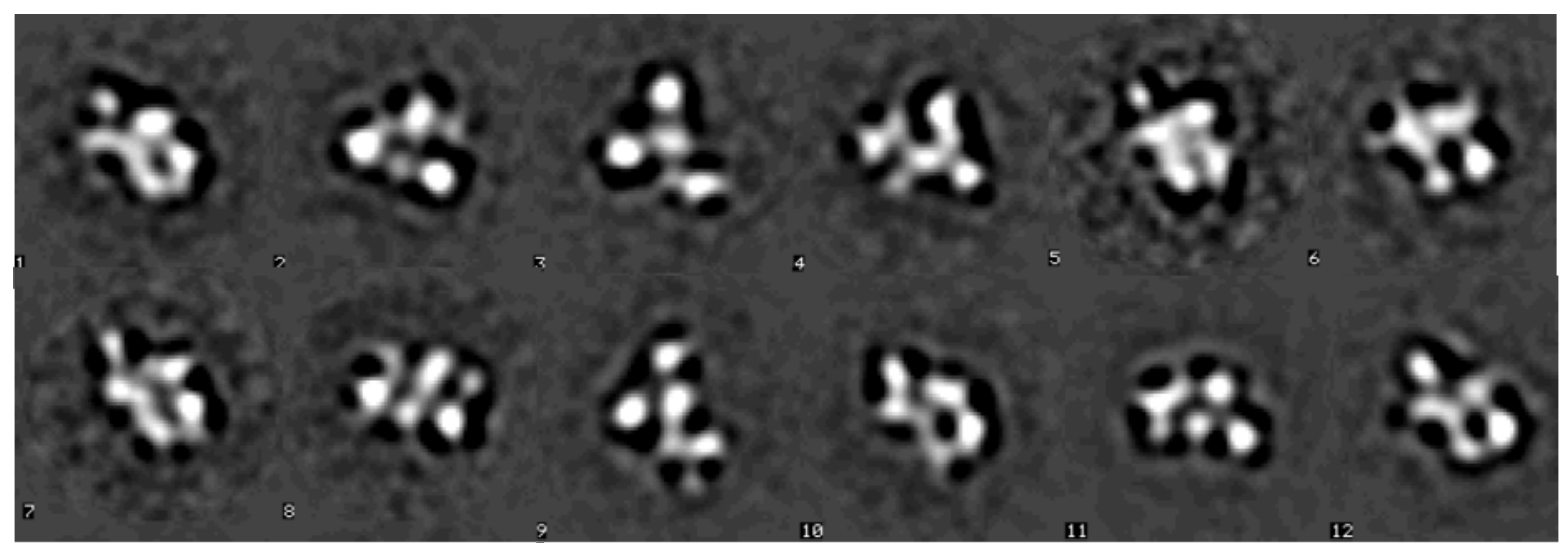

Figure 5.4. 2D analysis of the natively purified elF3. Multiple class averages of cryogenic samples of elF3 ${ }^{\text {nat }}$ show apparently 5 -lobed particles.

By initial comparison of the $2 \mathrm{D}$ class averages of $\mathrm{eIF} 3^{\text {nat }}$ and $3 \mathrm{D}$ reconstruction of $\mathrm{eIF} 3^{\mathrm{rec}}$, it appears that $2 \mathrm{D}$ projections of $\mathrm{eIF} 3^{\text {nat }}$ are akin to the back-projections of a monomer of eIF $3^{\text {rec }}$ reconstruction. This suggests the possibility of using the monomer of eIF $3^{\text {rec }}$ as the initial model for reconstruction of free or $40 \mathrm{~S}-$ bound eIF $3^{\text {nat }}$. Preliminary inspections of the $40 \mathrm{~S}-\mathrm{eIF} 3^{\text {rec }} 3 \mathrm{D}$ reconstruction revealed an extra density at the solvent-exposed side of the $40 \mathrm{~S}$ between the head 


\section{Discussion}

and the body which penetrates into the inter-subunit surface (Figure 5.5 C-E). Docking of the density of the model of yeast 40S subuinit (as a part of an 80S elongating ribosome; PDB code $1 \mathrm{~S} 1 \mathrm{H}$; Spahn et al. 2004) into the map of eIF $3^{\text {nat }}-40 \mathrm{~S}$ also suggests that this density most probably does not belong to the $40 \mathrm{~S}$ subunit. The density of eIF $3^{\text {rec }}$ monomer covers some parts of this extra density on eIF $3^{\text {nat }}-40 \mathrm{~S}$, so that it can be at least partially assigned to eIF3. Further data processing, both on eIF $3^{\text {rec }}$ monomer and eIF $3^{\text {nat }}-40 \mathrm{~S}$ is required to distinguish the other parts of eIF3 which are not currently well-resolved from the ribosome.

In order to gain further insights into the arrangement of the components of eIF3, its different subcomplexes were reconstituted in vitro, including Tif32/Prt1/Nip1 and Tif32/Prt1/Nip1/Tif34. These structures will reveal the position of Tif34 and Tif35 (The missing particles) as well as Prt1 (their interacting particle). For defining the position of Nip1, eIF3 was reconstituted with GST-tagged Nip1. GST is a $27 \mathrm{kDa}$ protein which should appear on the EM reconstruction as a blub of density. Knowing positions of Nip1, Prt1, Tif34 and Tif35 one would be able to assign the remaining density to Tif32. Preliminary results in the case of ternary and quaternary complexes have been obtained which indicate differences in density compared to the full complex. However, further analysis is required to confidently determine the differences.

\subsection{Crystallization trials on multi-factor complex}

In order to gain atomic-level structural insights into the network of interactions within MFC, most of the components of the MFC including eIF3, eIF5, eIF2 $\alpha$ and eIF2 $\beta$ were tested for crystallization (see table 5.1 for a complete list of trials). Stable fragments obtained by limited proteolysis were also cloned, purified and tested for crystallization. Obtained crystals were eIF3b-RRM (which will be discussed later), yeast eIF3i (Tif34) and fruit fly eIF3i.

Crystals of yeast eIF3i were obtained in 25\% PEG4000, 100mM Tris-Hcl pH8.5 and $200 \mathrm{mM}$ $\mathrm{Li}_{2} \mathrm{SO}_{4}$ (Figure 5.6 A). These crystals were of low quality, and although diffracting the X-ray to $\sim 4 \AA$, were suffering from high mosaicity. Different strategies were used to improve the crystals including screening of a wide range of buffers and $\mathrm{pHs}$, replacing $\mathrm{Li}_{2} \mathrm{SO}_{4}$ with different salts, using other PEGs, micro-seeding, screening different additives, methylation of surface-exposed lysines and crystallization at different temperatures. Also two truncations of Tif34 were designed, lacking one or two putative C-terminal helices. Despite all the efforts, crystals could not be improved considerably. 
A

B
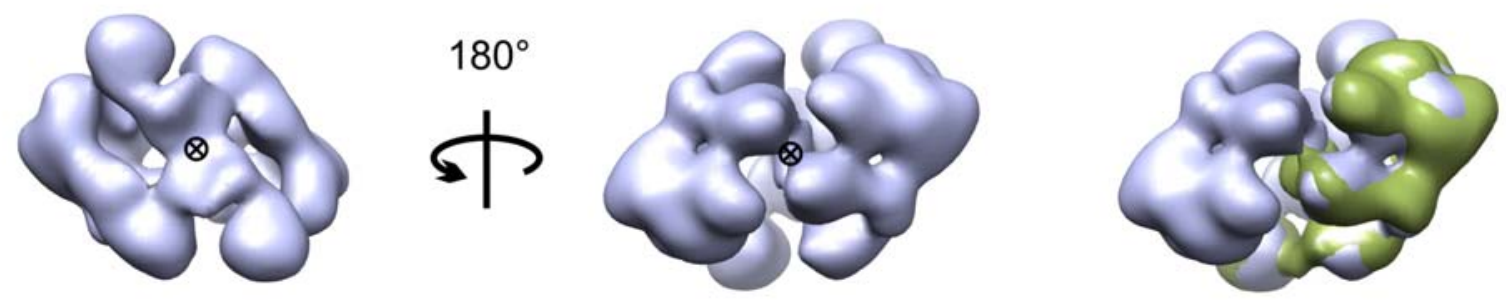

C
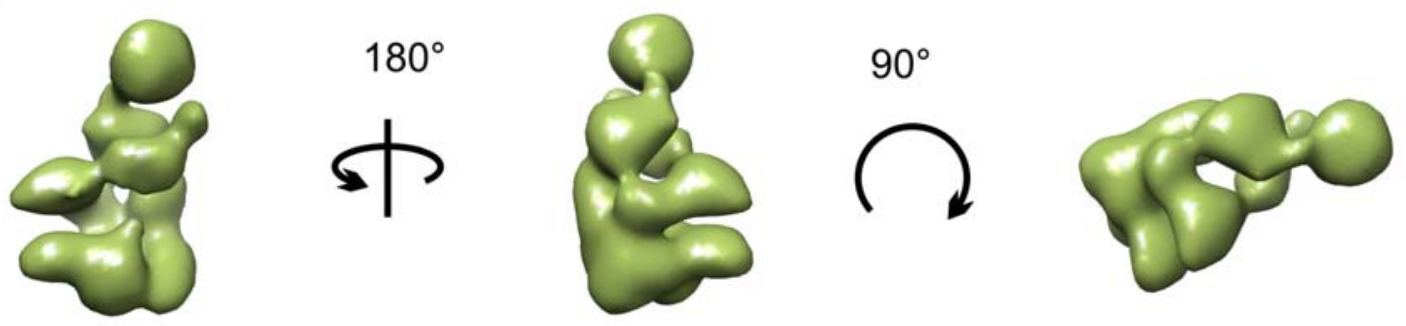

D

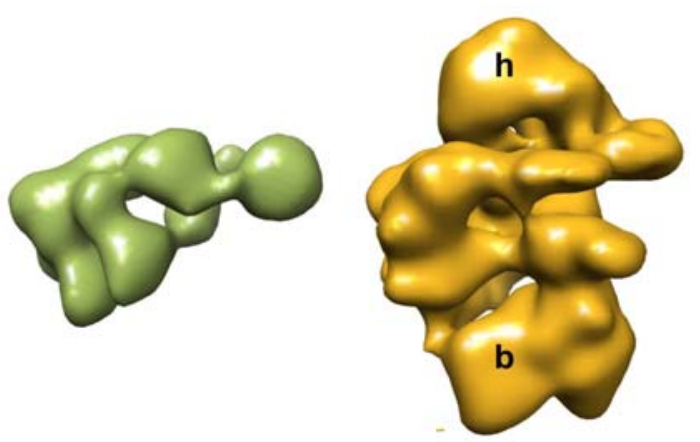

E
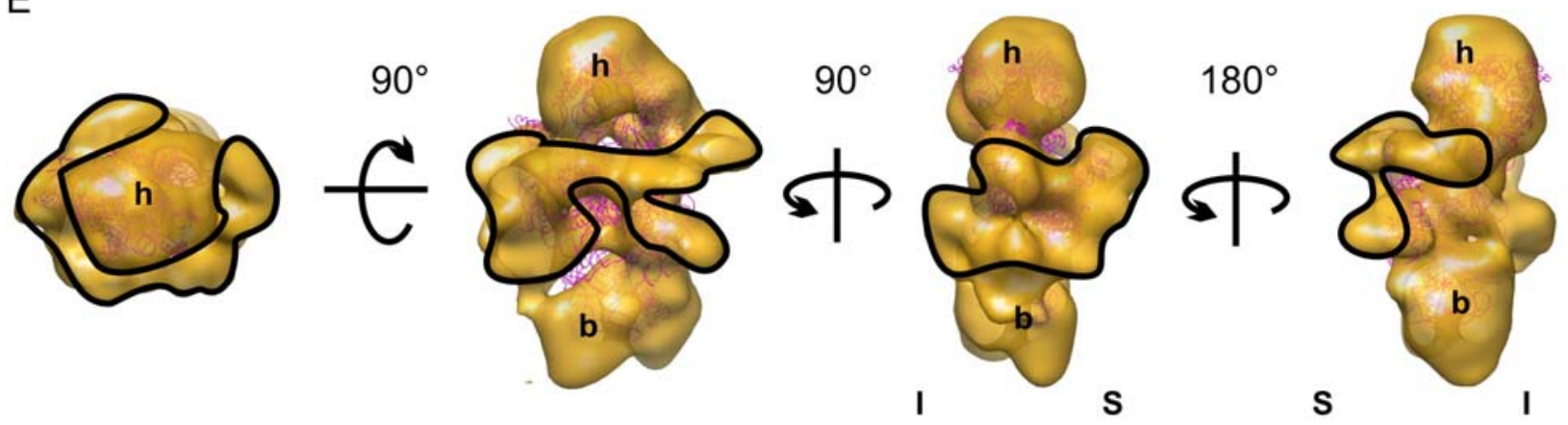

Figure 5.5. Preliminary 3D reconstructions of $40 \mathrm{~S}$-elF3 ${ }^{\text {nat }}$ and elF $3^{\text {rec }}$. (A) Two opposite faces of elF3 $3^{\text {rec }}$ dimer. The position of the two-fold symmetry is marked. (B) The dimer of elf $3^{\text {rec }}$ into which a monomer (green) is placed. This monomer was used as a search model for the extra density on the elF3 ${ }^{\text {rec }}-40 \mathrm{~S}$. (C) Three different views of the monomer of elF $3^{\text {rec }}$ which is extracted from the dimer reconstruction. (D) Solvent-exposed view of the elF3 ${ }^{\text {nat }}-40 \mathrm{~S}$ (yellow) aligned with the elF3 ${ }^{\text {rec }}$ (green) in a way that elF3 $3^{\text {rec }}$ covers the extra density visible between the head and the body of the 40S. (E) The model of yeast $40 \mathrm{~S}$ (purple ribbon, PDB code $1 \mathrm{~S} 1 \mathrm{H}$ ) docked into the map of elF $3^{\text {nat }}-40 \mathrm{~S}$. The position of the extra density is surrounded by black line. $\mathrm{H}, \mathrm{b}, \mathrm{I}$ and s stand for head, body, intersubunit face and solvent-exposed face, respectively. 


\section{Discussion}

In order to exploit the natural protein sequence variety between different organisms, crystallization of eIF3i from Drosophila melanogaster was tried. The construct (D. mel.-eIF3i in pGEX6-P1) was kindly provided by Jan Medenbach, group of Prof. Hentze, EMBL, Heidelberg. The protein was purified together with the bound GST-tag since the cleavage site of the PreScission protease was not accessible. GST-eIF3i was crystallized in a condition with 55\% MPD and $100 \mathrm{mM}$ Hepes $\mathrm{pH}$ 7. Fine-screening together with use of additive screens produced big crystals in 60\% MPD, $100 \mathrm{mM}$ Hepes pH 6.6 and 0.01M L-proline (Figure 5.6 B). However, the best diffracting crystals diffracted only to 5-6 $\AA$. In case of a well-diffracting crystal, presence of a GST bound to eIF3i, together with the fact that eIF3i most probably forms a sevenbladed $\beta$-propeller increases the chance of solving the structure by means of molecular replacement.

A

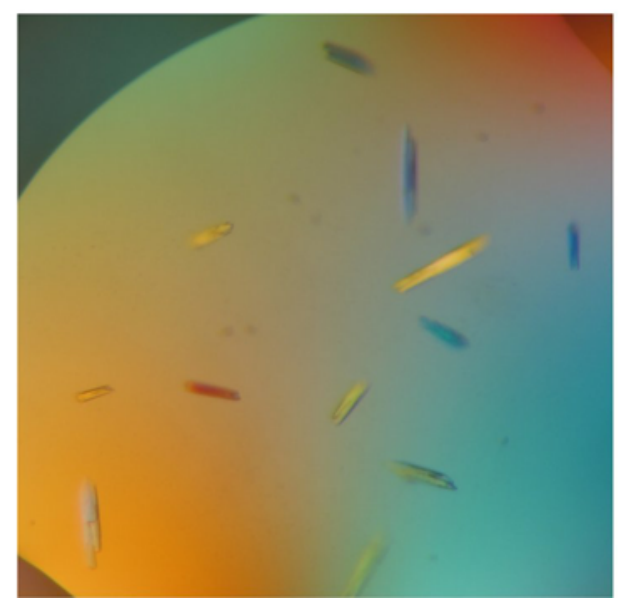

B

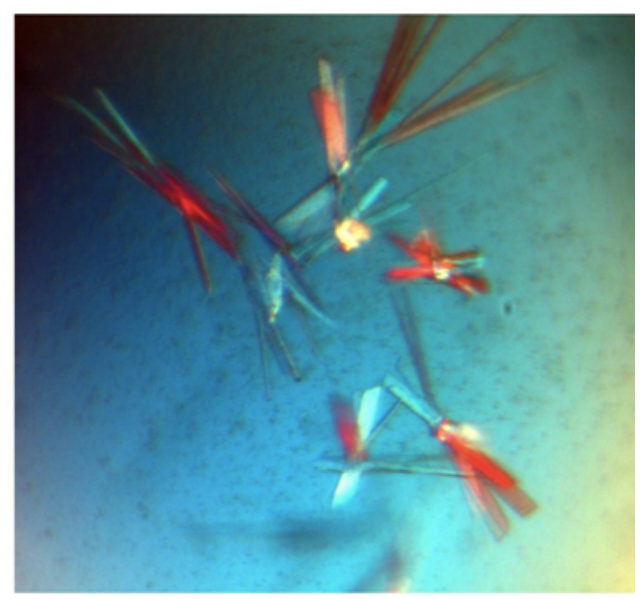

Figure 5.6. Crystals of elF3i. (A) Crystals of yeast elF3i (Tif34) grown 25\% PEG4000, 100mM Tris-Hcl pH8.5 and $200 \mathrm{mM} \mathrm{Li}_{2} \mathrm{SO}_{4}$. (B) Crystals of Drosophila melanogaster elF3i grown in 60\% MPD, $100 \mathrm{mM}$ Hepes pH 6.6 and $0.01 \mathrm{M} \mathrm{L-proline.} \mathrm{Both} \mathrm{of} \mathrm{these} \mathrm{crystal} \mathrm{forms} \mathrm{are} \mathrm{of} \mathrm{low} \mathrm{diffraction} \mathrm{quality.}$

\subsection{Crystal structure of yeast eIF3b-RRM}

Prt1/eIF3b plays critical roles in maintaining the integrity of eIF3 and mediating its ribosome binding activity. These roles are achieved via interactions of Prt1 N-terminal RNA recognition motif (RRM) with the C-terminal domain of Tif32/eIF3a as well as the loosely associated subunit Hcr1/eIF3j, respectively (Valásek et al. 2001)(Nielsen et al. 2006).

The structure of yeast eIF3b -RRM shows high similarity to other RRMs, with four strands and two helices arranged in a $\beta-\alpha-\beta-\beta-\alpha-\beta$ sequential order (Figure 3.6). The eIF3j-interacting surface of yeast eIF3b-RRM showed the same features as its human ortholog. The same mode of 


\section{Discussion}

interactions as in human governs the yeast eIF3b-RRM binding to eIF3j, owing to the fact that the thermodynamics of the binding were quite similar in both cases. The RNPs 1 and 2 in this structure are located on a basic or hydrophobic environment at the surface, making them suitable for oligonucleotide binding. The proposed RNA binding activity was confirmed by showing the interaction of yeast eIF3b-RRM with whole RNA extract of yeast (Khoshnevis, Neumann and Ficner 2010). As the majority of the yeast whole-RNA extract is rRNA, it is alluring to hypothesize that the almost complete shift of the yeast eIF3b-RRM seen in figure 3.8 is partly resulted from its binding to the rRNA. Nonetheless, the possibility that this domain helps eIF3 to recruit the mRNA to the ribosome can not be ruled out. In fact, the mRNA recruitment in yeast is impaired by depletion of eIF3 or eIF2 and seems to be to some extent independent of eIF4G (Jivotovskaya et al. 2006). Replacement of RNP1 motif of yeast eIF3b-RRM with a stretch of alanines has shown to impair its interaction with Hcrl and the binding to the ribosome (Nielsen et al. 2006). Knowing that this motif is not directly involved in Hcr1 binding, impairment of the ribosome binding activity can point to the main function of the RNP1 motif in interaction with either mRNA or rRNA, hence recruitment of the eIF3 to the ribosome.

Interestingly, the RRM domain of human eIF3b was recently shown to be engaged with domain III of HCV-IRES with ten times higher affinity than for eIF3j. (Pérard et al. 2009). These IRESinteracting regions, however, are spatially close to each other, being accumulated in a loop close to the eIF3j binding site. Therefore the interactions of HCV IRES with human eIF3b-RRM would compete and substitute those of eIF3j. This would explain how eIF3 is redirected from translation of cellular mRNA towards translation of HCV IRES RNA. However, this is not a usual mode of oligonucleotide binding for RRMs. The canonical RNA binding surface of human eIF3b-RRM is, as mentioned, negatively charged and not capable of interaction with RNA. It is noteworthy that no cellular-RNA binding activity has so far been reported for human eIF3b, indicating that HCV IRES element has evolved sequence specificities for hijacking eIF3b-RRM.

The difference in RNA binding capability of eIF3b-RRM between human and yeast at first glance seems to be at odds with the high conservation of eIF3 function among eukaryotes. However, human eIF3 has seven more subunits than yeast (eIF3d, e, f, h, k, 1, m). Two of these subunits, eIF3d and eIF3f have been proposed to have RNA binding activity (Asano et al. 1997). Apart from eIF3b, among other common eIF3 subunits between human and yeast, eIF3a, eIF3c and eIF3g are also suggested to have RNA binding activities (Hinnebusch 2006). Therefore, it is tempting to speculate that the missing cellular-RNA binding activity of human eIF3b might have been conferred to another, perhaps one of the human-specific, subunits. eIF3a and eIF3d, for 


\section{Discussion}

instance, have been cross-linked to the sequences upstream of AUG start codon in the 48S PIC (Pisarev et al. 2008).

\subsection{Rli1 has dual functions in translation initiation and termination}

In addition to playing the role in initiation, eIF3 has been engaged in the dissociation of posttermination $80 \mathrm{~S}$ ribosome. During the course of this work, a new function in translation termination was assigned to one of the eIF3-interacting proteins, Rli1.

Some general functions have been reported for Rlil including its involvements in ribosomal biogenesis as well as protein translation. Rli1p has been shown to interact with eIF2, eIF5 and multiple subunits of eIF3 in yeast (Dong et al., 2004). Rli1 was shown to interact with Sup45, a eukaryotic release factor, which in a complex with Sup35, induces the hydrolysis of the ester bond of the P-site peptidyl-tRNA. The interaction site for Sup45 on Rli1 was dissected on its second $\mathrm{ABC}$ domain. Further, the functional role of Rlil in translation termination was uncovered.

The fundamental role of Rlilp in RNase L inhibition, HIV capsid maturation, translation initiation, and ribosome biogenesis led Tampe and co-workers to suggest a role for two essential diamagnetic $[4 \mathrm{Fe}-4 \mathrm{~S}]^{2+}$ clusters in recognition and modification of RNA assemblies (Barthelme et al., 2007). The fact that deletion of Rli1's Fe-S clusters did not abolish its interaction with eRF1 and eIF3j is not contrary with the proposed function of Rli1. However, a more fundamental role for the Fe-S cluster concerning a signalling between the level of energy metabolism in mitochondria and the general rate of protein synthesis in the cytosol can not be ruled out.

The surprising involvement of Rlil in translation termination discussed here might have more implications. Since there is no apparent input of energy in eukaryotic recycling compared to the prokaryotic one, it was suggested that there is an auxiliary protein which has a rate-enhancing role involving hydrolysis of ATP or GTP in eukaryotic ribosomal recycling (Jackson 2007). The fact that Rlil belongs to the ABC family of proteins known for their ATPase activity together with its dual interaction with eIF3 (as the main recycling factor) and eRF1 (as the main terminating factor) sets it forth as a putative candidate. In agreement with our hypothesis, Pestova and colleagues have shown that ribosome recycling in a wide range of $\mathrm{Mg}^{2+}$ concentrations requires $\mathrm{ABCE} 1$, the mammalian homologue of Rli1, which dissociates posttermination complexes (post-TCs) into free 60S subunits and mRNA- and tRNA-bound 40S subunits. To exert its function, ABCE1 hydrolyzes ATP, GTP, UTP and CTP, thus providing the 


\section{Discussion}

source of energy for dissociation of the subunits (Pisarev et al. 2010). In yeast cytoplasm, eukaryotic elongation factor 3 (eEF3), a protein found in yeast and other fungi but missing in higher eukaryotes, disassembles post-translational ribosomes into their subunits, mRNA and tRNA in an ATP dependant manner (Kurata et al.2010). eEF3 was known before to be an E-site factor with ribosome-dependant ATPase activity which facilitates the release of the E-site deacylated tRNA (Triana-Alonso et al. 1995). Interestingly, eEF3 also belongs to the ABC superfamily of ATPases and has been shown to bind to the ribosome in the ADP- or apo-form at a position covering both subunits near the $\mathrm{E}$ site. This binding induces movement of L1 stalk to the "out" state upon binding and hydrolysis of ATP, facilitating the release of E-site tRNA (Andersen et al. 2006). However, one could speculate that upon termination and binding of eRF1/eRF3 to the A-site, ribosome may undergo conformational changes which would lead to the dissociation of the subunits by ATP-induced tweezers-like movement of ABC domain of eEF3.

The fact that eEF3 was shown to serve the post-TC disassembly function in yeast does not exclude Rli1 from performing the same function. It is worth mentioning that Kaji's group also proposed that eEF2/ATP dissociates 80S ribosome into subunits (Demeshkina et al. 2007). However, this protein had no effect on post-TC (Kurata et al.2010). Therefore, it is alluring to speculate that different factors may perform the same function perhaps under different cellular conditions. The interaction site of eEF3 on the ribosome to some extent covers the A- and Psites. If we assume that Rli1 would also serve such a function as eEF3 and binds the same place, this positioning would be ideal for its observed interaction with eRF1 and eRF3.

This data therefore suggests a connection between translation initiation, termination and ribosomal recycling. Despite some ambiguities, eIF3 seems to be involved in all these processes. The fact that Pestova's group used a short RNA to form the post-TC is somewhat reminiscent of the short uORFs of some mRNAs. eIF3 is shown to stay bound to the ribosome for few cycles of elongation which is the key for its role in re-initiation after such short uORFs. Therefore, it's ' $\mathrm{j}$ ' subunit might in a way recruit ABCE1/Rli1 to the post-TC and induce its disassembly. The fact that eEF3 has an extra HEAT domain, a four helix bundle and a chromodomain compared to Rli1/ABCE1 may account for its apparent eIF3-independent association with the ribosome. Hence, recruitment of Rli1/ABCE1 to the long "native" mRNAs may require its interaction with release factors, as observed in our study. 
Table 5.1. List of all truncations which were tested for crystallization. The left column represents the full length subunits, either in isolation or in sub-complexes, and the right column lists their corresponding truncations. * Prt1 ${ }^{181 C}$ is the exception in the left column, as it represents most of Prt1 and is a stable product during Prt1 purification. + represents truncations which could not purified alone, however were present in the complex with other factors.

\begin{tabular}{|c|c|}
\hline Tif34 & Tif34 ${ }^{1-330}$ and Tif34 $4^{1-344}$ \\
\hline Tif35 & Tif35 $5^{14-150}+$ \\
\hline Tif34/Tif35 & Tif34/Tif35 ${ }^{14-150}$ \\
\hline Prt1 + & $\operatorname{Prt1}^{74-170}$ and Prt1 ${ }^{181 C}$ and Prt1 $1^{670 C}$ \\
\hline $\operatorname{Prt1}^{181 C} * /$ Tif34 & Tif34/Prt1 ${ }^{670 C}$ \\
\hline $\operatorname{Prt1}^{181 \mathrm{C}} /$ Tif34/Tif35 & Tif35 $5^{14-150} /$ Tif34/ Prt1 ${ }^{670 \mathrm{C}}$ \\
\hline Tif32 & Tif32 $2^{1-578}$ and Tif32 $2^{1-494}$ \\
\hline Nip1 & $\mathrm{Nip} 1^{240 \mathrm{C}}$ and $\mathrm{Nip} 1^{244 \mathrm{C}}$ \\
\hline & Tif32 $2^{1-578} / \mathrm{Nip}^{240 \mathrm{C}}$ and Tif32 $2^{1-494} / \mathrm{Nip}^{240 \mathrm{C}}$ \\
\hline II3य/NIpI & Tif32 $2^{1-578} / \mathrm{Nip}^{244 \mathrm{C}}$ and Tif32 $2^{1-494} / \mathrm{Nip} 1^{244 \mathrm{C}}$ \\
\hline Tif32/Nip1/Prt1 & Tif32/Nip1/Prt1 ${ }^{181 \mathrm{C}}$ \\
\hline elF2 $\alpha$ & elF $2 \alpha^{1-290}$ \\
\hline elF2 $\beta$ & elF $2 \beta^{125 c}$ \\
\hline elF5 & elF5 ${ }^{201 C}$ and elF5 $5^{241 C}$ \\
\hline elF5/ elF2 $\beta$ & $\mathrm{elF5}^{201 c} / \mathrm{elF} 2 \beta$ and elF5 ${ }^{241 c} / \mathrm{elF} 2 \beta$ \\
\hline Hcr1 & Hcr1/Prt1 $1^{74-170}$ \\
\hline elF1 & \\
\hline elF4B & \\
\hline
\end{tabular}




\section{Chapter 6 • Summary and Future Perspectives}

eIF3 plays a central role in eukaryotic translation initiation. Its complexity has so far hampered its purification, in vitro reconstitution and structural and kinetic studies. The main part of this work was dedicated to establish protocols for recombinantly producing its components and reconstituting them in the form of an active complex in vitro. The complex obtained was shown to be active as it binds to the 40S ribosomal subunit. However, purification of Tif32 and Nip1 alone yielded much more pure samples. Therefore, establishing new protocol for purification of Prt1, the third large subunit of eIF3, seem to be pivotal for reconstitution of an absolutely pure eIF3-complex devoid of any undesirable co-purification of truncated fragments.

Limited proteolysis obtained different truncations of the subunits which could form subcomplexes. Although several hundred crystallization conditions have already been screened for crystallization, another round of trials using our new state-of-the-art crystallization facility by varying more parameters is going to be the next step towards crystallization of parts of eIF3.

Initial EM analyses of the complex revealed an overall globular shape of the complex with some degrees of symmetry. The apparent molecular weight of the complex, consistent with the gelfiltration elution profile, suggests that some of the subunits must be present in more than one copy. Further analysis of the subcomplexes of eIF3, depleted in different subunits, as well the GST-tagged complex will reveal the structural arrangement of different subunits within the complex. To achieve this, we will benefit from the structural rigidity of $40 \mathrm{~S}$ ribosome when bound to natively purified eIF3 in order to reconstruct the structure of native eIF3, and from that, reconstituted eIF3 and its subcomplexes.

Fluorescence-labeling of Tif32/eIF3a, the largest subunit of eIF3, provides new strategies to dissect the kinetics of translation initiation in yeast. We had the first insights into the complex formation between two core subunits of eIF3, Tif32/3a and Nip1/3c. In addition, we observed a different behavior of Tif32/3a dissociation in the absence or presence of the $40 \mathrm{~S}$ ribosomal subunit. We cannot, however, judge too much based on the obtained results. In order to improve the observations, the labeling efficiency of Tif32/3a should be increased by screening more conditions and/or different fluorophores. In addition, based on the previous reports on the fluorescence-labeling of other components of initiation, we have to search for suitable conditions to form FRET pairs between eIF3 and other initiation factors such as eIF1 and eIF1A.

The RNA recognition motif of Prt1/3a was shown to be a canonical RRM, having RNA binding 
activity. Determination of its exact RNA substrate, however, was out of the scope of this project. Nonetheless, future experiments are required to address its RNA target. Using in vitro reconstitution protocol established here and assembling the complex either harboring some key mutation in this domain or completely lacking it, followed by kinetic studies would precisely establish its role during translation initiation.

Finally, a new role for Rli1/ABCE1, a member of ABC superfamily of ATPases was found in translation termination. It was shown to interact with other termination factors, eRF1 and eRF3 and play a role in decoding of the stop codon. The interesting finding that in human this protein plays a crucial role in ribosome recycling may suggest the same role for Rli1 in yeast, perhaps under different conditions than the recently discovered recycling factor, EF3. 


\section{Chapter $7 \bullet$ Bibliography}

Acker, M.G., Kolitz, S.E., Mitchell, S.F., Nanda, J.S. and Lorsch, J.R. 2007. Reconstitution of Yeast Translation Initiation. Methods in Enzymology 430: 111-145.

Acker, M.G., Shin, B., Dever, T.E., and Lorsch, J.R. 2006. Interaction between eukaryotic initiation factors $1 \mathrm{~A}$ and $5 \mathrm{~B}$ is required for efficient ribosomal subunit joining. The Journal of biological chemistry 281: 8469-75.

Acker, M.G., Shin, B., Nanda, J.S., Saini, A.K., Dever, T.E., and Lorsch, J.R. 2009. Kinetic analysis of late steps of eukaryotic translation initiation. Journal of molecular biology 385: 491506.

Adams, P.D., Afonine, P.V., Bunkoczi, G., Chen, V.B., Davis, I.W., Echols, N., Headd, J.J., Hung, L.W., Kapral, G.J., Grosse-Kunstleve, R.W., McCoy, A.J., Moriarty, N.W., Oeffner, R., Read, R.J., Richardson, D.C., Richardson, J.S., Terwilliger, T.C., and Zwart, P.H. 2010. PHENIX: a comprehensive Python-based system for macromolecular structure solution. Acta crystallographica. Section D, Biological crystallography 66: 213-21.

Algire, M.a., Maag, D., and Lorsch, J.R. 2005. Pi release from eIF2, not GTP hydrolysis, is the step controlled by start-site selection during eukaryotic translation initiation. Molecular cell 20: 251-62.

Andersen, C.B., Ballut, L., Johansen, J., Chamieh, H., Nielsen, K.H., Oliveira, C.L.P., Pedersen, J.S., Seraphin, B., Le Hir, H., and Andersen, G.R. 2006. Structure of the exon junction core complex with a trapped DEAD-box ATPase bound to RNA. Science 313: 1968-72.

Andersen, G.R., Valente, L., Pedersen, L., Kinzy, T.G., and Nyborg, J. 2001. Crystal structures of nucleotide exchange intermediates in the eEF1A-eEF1Balpha complex. Nature structural biology 8: 531-4.

Asano, K., Kinzy, T.G., Merrick, W.C., and Hershey, J.W. 1997. Conservation and diversity of eukaryotic translation initiation factor eIF3. The Journal of biological chemistry 272: 1101-9.

Asano, K., Krishnamoorthy, T., Phan, L., Pavitt, G.D., and Hinnebusch, A.G. 1999. Conserved bipartite motifs in yeast eIF5 and eIF2Bepsilon, GTPase-activating and GDP-GTP exchange factors in translation initiation, mediate binding to their common substrate eIF2. The EMBO journal 18: 1673-88.

Asano, K., Phan, L., Anderson, J., and Hinnebusch, A.G. 1998. Complex formation by all five homologues of mammalian translation initiation factor 3 subunits from yeast Saccharomyces cerevisiae. The Journal of biological chemistry 273: 18573-85.

Asano, K., Shalev, a., Phan, L., Nielsen, K., Clayton, J., Valásek, L., Donahue, T.F., and Hinnebusch, A.G. 2001. Multiple roles for the C-terminal domain of eIF5 in translation initiation complex assembly and GTPase activation. The EMBO journal 20: 2326-37.

Asano, K., Vornlocher, H.P., Richter-Cook, N.J., Merrick, W.C., Hinnebusch, A.G., and Hershey, J.W. 1997. Structure of cDNAs encoding human eukaryotic initiation factor 3 subunits. 
Possible roles in RNA binding and macromolecular assembly. The Journal of biological chemistry 272: 27042-52.

Asano, K. 2000. A multifactor complex of eukaryotic initiation factors, eIF1, eIF2, eIF3, eIF5, and initiator tRNAMet is an important translation initiation intermediate in vivo. Genes \& development 14: 2534-2546.

Battiste, J.L., Pestova, T.V., Hellen, C.U., and Wagner, G. 2000. The eIF1A solution structure reveals a large RNA-binding surface important for scanning function. Molecular cell 5: 109-19.

Berchtold, H., Reshetnikova, L., Reiser, C.O., Schirmer, N.K., Sprinzl, M. and Hilgenfeld, R. 1993. Crystal structure of active elongation factor $\mathrm{Tu}$ reveals major domain rearrangements. Nature 365:126-32.

Bhattacharya, A., Czaplinski, K., Trifillis, P., He, F., Jacobson, A., and Peltz, S.W. 2000 Characterization of the biochemical properties of the human Upf1 gene product that is involved in nonsense-mediated mRNA decay. RNA 6, 1226-1235.

Bidou, L., Stahl, G., Hatin, I., Namy, O., Rousset, J.P., and Farabaugh, P.J. 2000 Nonsensemediated decay mutants do not affect programmed -1 frameshifting. RNA 6, 952-961.

Bieniossek, C., Schütz, P., Bumann, M., Limacher, A., Uson, I., and Baumann, U. 2006. The crystal structure of the carboxy-terminal domain of human translation initiation factor eIF5. Journal of molecular biology 360: 457-65.

Bisbal, C., Martinand, C., Silhol, M., Lebleu, B., and Salehzada, T. 1995 Cloning and characterization of a RNAse L inhibitor. A new component of the interferon-regulated 2-5A pathway. The Journal of Biological chemistry 270, 13308-13317.

Bolger, T.A., Folkmann, A.W., Tran, E.J., and Wente, S.R. 2008 The mRNA export factor Gle1 and inositol hexakisphosphate regulate distinct stages of translation. Cell 134, 624-633.

Borman, A.M., Michel, Y.M., and Kean, K.M. 2000. Biochemical characterisation of cappoly(A) synergy in rabbit reticulocyte lysates: the eIF4G-PABP interaction increases the functional affinity of eIF4E for the capped mRNA 5'-end. Nucleic acids research 28: 4068-75.

Chen, Z., Dong, J., Ishimura, A., Daar, I., Hinnebusch, A.G., and Dean, M. 2006. The essential vertebrate ABCE1 protein interacts with eukaryotic initiation factors. The Journal of biological chemistry 281: 7452-7.

Cho, S., and Hoffman, D.W. 2002. Structure of the $\beta$ Subunit of Translation Initiation Factor 2 from the Archaeon Methanococcus jannaschii : A Representative of the eIF2 $\beta /$ eIF5 Family of Proteins. Biochemistry 41: 5730-5742.

Cigan, A.M., Pabich, E.K., Feng, L., and Donahue, T.F. 1989. Yeast translation initiation suppressor sui2 encodes the alpha subunit of eukaryotic initiation factor 2 and shares sequence identity with the human alpha subunit. Proceedings of the National Academy of Sciences of the United States of America 86: 2784-8.

Cléry, A., Blatter, M., and Allain, F.H. 2008. RNA recognition motifs: boring? Not quite. Current opinion in structural biology 18: 290-8. 
Cole, C.N. and Scarcelli, J.J. 2006 Transport of messenger RNA from the nucleus to the cytoplasm. Current Opinion in Cell Biology 18, 299-306.

Conte, M.R., Kelly, G., Babon, J., Sanfelice, D., Youell, J., Smerdon, S.J., and Proud, C.G. 2006. Structure of the eukaryotic initiation factor (eIF) 5 reveals a fold common to several translation factors. Biochemistry 45: 4550-8.

Crowder, S.M., Kanaar, R., Rio, D.C., and Alber, T. 1999. Absence of interdomain contacts in the crystal structure of the RNA recognition motifs of Sex-lethal. Proceedings of the National Academy of Sciences of the United States of America 96: 4892-7.

Cullen, R.B. 2009. Viral RNAs: Lessons from the Enemy. Cell 136(4): 592-97.

Dallas, a., and Noller, H.F. 2001. Interaction of translation initiation factor 3 with the $30 \mathrm{~S}$ ribosomal subunit. Molecular cell 8: 855-64.

Damoc, E., Fraser, C.S., Zhou, M., Videler, H., Mayeur, G.L., Hershey, J.W., Doudna, J.a., Robinson, C.V., and Leary, J.A. 2007. Structural characterization of the human eukaryotic initiation factor 3 protein complex by mass spectrometry. Molecular \& cellular proteomics $\mathbf{6}$ : 1135-46.

Davis, I.W., Murray, L.W., Richardson, J.S., and Richardson, D.C. 2004. MOLPROBITY: structure validation and all-atom contact analysis for nucleic acids and their complexes. Nucleic acids research 32: 615-9.

de Melo Neto, O.P., Standart, N., and Martins de Sa, C. 1995. Autoregulation of poly(A)binding protein synthesis in vitro. Nucleic acids research 23(12): 2198-205.

Demeshkina, N., Hirokawa, G., Kaji, A., and Kaji, H. 2007. Novel activity of eukaryotic translocase, eEF2: dissociation of the $80 \mathrm{~S}$ ribosome into subunits with ATP but not with GTP. Nucleic acids research 35: 4597-607.

Dessau, M., Halimi, Y., Erez, T., Chomsky-Hecht, O., Chamovitz, D.a., and Hirsch, J.A. 2008. The Arabidopsis COP9 signalosome subunit 7 is a model PCI domain protein with subdomains involved in COP9 signalosome assembly. The Plant cell 20: 2815-34.

Dhaliwal, S., and Hoffman, D.W. 2003. The Crystal Structure of the N-terminal Region of the Alpha Subunit of Translation Initiation Factor 2 (eIF2 $\alpha$ ) from Saccharomyces cerevisiae Provides a View of the Loop Containing Serine 51, the Target of the eIF2 $\alpha$-specific Kinases. Journal of Molecular Biology 334: 187-195.

Diaconu, M., Kothe, U., Schlünzen, F., Fischer, N., Harms, J.M., Tonevitsky, A.G., Stark, H., Rodnina, M.V., and Wahl, M.C. 2005. Structural basis for the function of the ribosomal L7/12 stalk in factor binding and GTPase activation. Cell 121: 991-1004.

Ding, J., Hayashi, M.K., Zhang, Y., Manche, L., Krainer, a.R., and Xu, R. 1999. Crystal structure of the two-RRM domain of hnRNP A1 (UP1) complexed with single-stranded telomeric DNA. Genes \& development 13: 1102-1115.

Donahue, T.F., and Cigan, A.M. 1988. Genetic selection for mutations that reduce or abolish ribosomal recognition of the HIS4 translational initiator region. Molecular and cellular biology 8: $2955-63$. 
Dong, J., Lai, R., Nielsen, K., Fekete, C.A., Qiu, H., and Hinnebusch, A.G. 2004. The essential ATP-binding cassette protein RLI1 functions in translation by promoting preinitiation complex assembly. The Journal of biological chemistry 279: 42157-68.

Elantak, L., Tzakos, A.G., Locker, N., and Lukavsky, P.J. 2007. Structure of eIF3b RNA recognition motif and its interaction with eIF3j: structural insights into the recruitment of eIF3b to the $40 \mathrm{~S}$ ribosomal subunit. The Journal of biological chemistry 282: 8165-74.

Elantak, L., Wagner, S., Herrmannová, A., Karásková, M., Rutkai, E., Lukavsky, P.J. and Valásek, L. 2010. The indispensable N-terminal half of eIF3j/HCR1 cooperates with its structurally conserved binding partner eIF3b/PRT1-RRM and with eIF1A in stringent AUG selection. Journal of molecular biology. 396: 1097-116.

Emsley, P., and Cowtan, K. 2004. Coot: model-building tools for molecular graphics. Acta crystallographica. Section D, Biological crystallography 60: 2126-32.

Erickson, F.L., Nika, J., Rippel, S., and Hannig, E.M. 2001. Minimum requirements for the function of eukaryotic translation initiation factor 2. Genetics 158: 123-32.

Evans, P. 2006. Scaling and assessment of data quality. Acta crystallographica. Section D, Biological crystallography 62: 72-82.

Fabian, M.R. Mathonnet, G., Sundermeier, T., Mathys, H., Zipprich, J.T., Svitkin, Y.V., Rivas, F., Jinek, M., Wohlschlegel, J., Doudna, J.A., Chen, C.A., Shyu, A., Yates III, J.R., Hannon, G.J. Filipowicz, W., Duchaine, T.F., and Sonenberg, N. 2009. Mammalian miRNA RISC recruits CAF1 and PABP to affect PABP-dependent deadenylation. Molecular cell 35: 868-80.

Fraser, C.S., Berry, K.E., Hershey, J.W., and Doudna, J.A. 2007. eIF3j is located in the decoding center of the human 40S ribosomal subunit. Molecular cell 26: 811-9.

Fringer, J.M., Acker, M.G., Fekete, C.a., Lorsch, J.R., and Dever, T.E. 2007. Coupled release of eukaryotic translation initiation factors $5 \mathrm{~B}$ and $1 \mathrm{~A}$ from $80 \mathrm{~S}$ ribosomes following subunit joining. Molecular and cellular biology 27: 2384-97.

Gouet, P., Courcelle, E., Stuart, D.I., and Métoz, F. 2000. BIOINFORMATICS ESPript : analysis of multiple sequence alignments in PostScript. Bioinformatics 15: 305-308.

Gross, T., Siepmann, A., Sturm, D., Windgassen, M., Scarcelli, J.J., Seedorf, M., Cole, C.N. and Krebber, H. 2007 The DEAD-box RNA helicase Dbp5 functions in translation termination. Science 315, 646-649.

Gutiérrez, P., Osborne, M.J., and Siddiqui, N. 2004. Structure of the archaeal translation initiation factor Implications for translation initiation. Structure 659-667.

Hammond, S.M., Boettcher, S., Caudy, A.A., Kobayashi, R., and Hannon, G.J. 2001. Argonaute2, a link between genetic and biochemical analyses of RNAi. Science 293: 1146-50.

Handa, N., Nureki, O., Kurimoto, K., Kim, I., Sakamoto, H., Shimura, Y., Muto, Y., and Yokoyama, S. 1999. Structural basis for recognition of the tra mRNA precursor by the Sexlethal protein. Nature 398: 579-85. 
Hannig, E.M., Cigan, A.M., Freeman, B.A., and Kinzy, T.G. 1993. Negative Regulator of GCN4 Expression, Encodes Subunit of eIF-2 in Saccharomyces cerevisiae. Microbiology 13: 506-520.

Hengen, P.N. 1997 False positives from the yeast two-hybrid system. Trends in Biochemical Science 22, 33-34.

Hinnebusch, A.G. 2006. eIF3: a versatile scaffold for translation initiation complexes. Trends in biochemical sciences 31: 553-62.

Hutvágner, G., McLachlan, J., Pasquinelli, a.E., Bálint, E., Tuschl, T., and Zamore, P.D. 2001. A cellular function for the RNA-interference enzyme Dicer in the maturation of the let-7 small temporal RNA. Science 293: 834-8.

Ito, T., Marintchev, A., and Wagner, G. 2004. Solution structure of human initiation factor eIF2alpha reveals homology to the elongation factor eEF1B. Structure 12: 1693-704.

Jackson, R.J., Hellen, C.U., and Pestova, T.V. 2010. The mechanism of eukaryotic translation initiation and principles of its regulation. Nature reviews Molecular cell biology 11: 113-27.

Jacobson, A. 2005 The end justifies the means. Nature Structural and Molecular Biology 12, 474-475.

Jennings, M.D., and Pavitt, G.D. 2010. eIF5 has GDI activity necessary for translational control by eIF2 phosphorylation. Nature 465: 378-81.

Jivotovskaya, A.V., Valasek, L., Hinnebusch, A.G., and Nielsen, K.H. 2006. Eukaryotic Translation Initiation Factor 3 ( eIF3 ) and eIF2 Can Promote mRNA Binding to 40S Subunits Independently of eIF4G in Yeast. Gene 26: 1355-1372.

Kabsch, W. 2010. XDS. Acta crystallographica. Section D, Biological crystallography 66: 12532.

Kapp, L.D. and Lorsch, J.R. 2004a. GTP-dependent Recognition of the Methionine Moiety on Initiator tRNA by Translation Factor eIF2. Journal of Molecular Biology 335: 923-936.

Kapp, L.D. and Lorsch, J.R. 2004b The molecular mechanics of eukaryotic translation. Annual Reviews in Biochemistry 73, 657-704.

Kastner, B., Fischer, N., Golas, M.M., Sander, B., Dube, P., Boehringer, D., Hartmuth, K., Deckert, J., Hauer, F., Wolf, E., Uchtenhagen, H., Urlaub, H., Herzog, F., Peters, J.M., Poerschke, D., Luhrmann, R. and Stark, H. 2008. GraFix : sample preparation for single- particle electron cryomicroscopy. Nature Methods 5: 53-55.

Kavran, J.M. and Steitz, T.A. 2007. Structure of the base of the L7/L12 stalk of the Haloarcula marismortui large ribosomal subunit: analysis of L11 movements. Journal of molecular biology 371: $1047-59$.

Khoshnevis, S., Gross, T., Rotte, C., Baierlein, C., Ficner, R., and Krebber, H. 2010. The ironsulphur protein RNase L inhibitor functions in translation termination. EMBO reports 11: 214-9. 
Khoshnevis, S., Neumann, P., and Ficner, R. 2010. Crystal Structure of the RNA Recognition Motif of Yeast Translation Initiation Factor eIF3b Reveals Differences to Human eIF3b. PLoS ONE 5(9): e12784.

Kim, T., Hofmann, K., von Arnim, A.G., and Chamovitz, D.A. 2001. PCI complexes: pretty complex interactions in diverse signaling pathways. Trends in plant science 6: 379-86.

Kispal, G., Sipos, K., Fekete, Y., Bedekovics, T., Janaky, T., Bassler, J., Aguilar Netz, D.J., Balk, J., Rotte, C., and Lill, R. 2005. Biogenesis of cytosolic ribosomes requires the essential iron-sulphur protein Rli1p and mitochondria. The EMBO journal 24: 589-98.

Kolitz, S.E., Takacs, J.E., and Lorsch, J.R. 2009. Kinetic and thermodynamic analysis of the role of start codon/anticodon base pairing during eukaryotic translation initiation. $R N A$ 15: 138-52.

Kolupaeva, V.G., Unbehaun, A., Lomakin, I.B., Hellen, C.U., and Pestova, T.V. 2005. Binding of eukaryotic initiation factor 3 to ribosomal $40 \mathrm{~S}$ subunits and its role in ribosomal dissociation and anti-association. $R N A$ 11: 470-86.

Korneeva, N.L., Lamphear, B.J., Hennigan, F.L., and Rhoads, R.E. 2000. Mutually cooperative binding of eukaryotic translation initiation factor (eIF) 3 and eIF4A to human eIF4G-1. The Journal of biological chemistry 275: 41369-76.

Krissinel, E., and Henrick, K. 2007. Inference of macromolecular assemblies from crystalline state. Journal of molecular biology 372: 774-97.

Kuo, P., Doudeva, L.G., Wang, Y., Shen, C.J., and Yuan, H.S. 2009. Structural insights into TDP-43 in nucleic-acid binding and domain interactions. Nucleic acids research 37: 1799-808.

Kurata, S., Nielsen, K.H., Mitchell, S.F., Lorsch, J.R., Kaji, A., and Kaji, H. 2010. Ribosome recycling step in yeast cytoplasmic protein synthesis is catalyzed by eEF3 and ATP. Proceedings of the National Academy of Sciences 107: 10854-10859.

Larkin, M.A., Blackshields, G., Brown, N.P., Chenna, R., McGettigan, P.A., McWilliam, H., Valentin, F., Wallace, I.M., Wilm, A., Lopez, R., Thompson, J.D., Gibson, T.J., and Higgins, D.G. 2007. Clustal W and Clustal X version 2.0. Bioinformatics 23: 2947-8.

Le Roy, F., Salehzada, T., Bisbal, C., Dougherty, J.P. and Peltz, S.W. 2005 A newly discovered function for RNase L in regulating translation termination. Nature Structural and Molecular Biology 12, 505-512.

Li, D., and Roberts, R. 2001. Human Genome and Diseases : Review. WD-repeat proteins : structure characteristics, biological function, and their involvement in human diseases. Cellular and Molecular Life Sciences 58: 2085- 2097.

Lill, R. 2009 Function and biogenesis of iron-sulphur proteins. Nature 460, 831-838.

Lomakin, I.B., Kolupaeva, V.G., Marintchev, A., Wagner, G., and Pestova, T.V. 2003. Position of eukaryotic initiation factor eIF1 on the $40 \mathrm{~S}$ ribosomal subunit determined by directed hydroxyl radical probing. Genes \& development 17: 2786-97. 
Maag, D., Algire, M.A., and Lorsch, J.R. 2006. Communication between eukaryotic translation initiation factors 5 and $1 \mathrm{~A}$ within the ribosomal pre-initiation complex plays a role in start site selection. Journal of molecular biology 356: 724-37.

Maag, D., Fekete, C.A., Gryczynski, Z., and Lorsch, J.R. 2005. A conformational change in the eukaryotic translation preinitiation complex and release of eIF1 signal recognition of the start codon. Molecular cell 17: 265-75.

Maag, D. and Lorsch, J.R. 2003. Communication Between Eukaryotic Translation Initiation Factors 1 and 1A on the Yeast Small Ribosomal Subunit. Journal of Molecular Biology 330: 917-924.

Marcotrigiano, J., Gingras, A.C., Sonenberg, N., and Burley, S.K. 1999. Cap-dependent translation initiation in eukaryotes is regulated by a molecular mimic of eIF4G. Molecular cell 3: 707-16.

Marintchev, A., Edmonds, K.A., Marintcheva, B., Hendrickson, E., Oberer, M., Suzuki, C., Herdy, B., Sonenberg, N., and Wagner, G. 2009. Topology and regulation of the human eIF4A/4G/4H helicase complex in translation initiation. Cell 136: 447-60.

Marintchev, A., Kolupaeva, V.G., Pestova, T.V., and Wagner, G. 2003. Mapping the binding interface between human eukaryotic initiation factors 1A and 5B: a new interaction between old partners. Proceedings of the National Academy of Sciences of the United States of America 100: $1535-40$.

Martin-Tumasz, S., Reiter, N.J., Brow, D.A., and Butcher, S.E. 2010. Structure and functional implications of a complex containing a segment of U6 RNA bound by a domain of Prp24. RNA 16: $792-804$.

Masutani, M., Sonenberg, N., Yokoyama, S., and Imataka, H. 2007. Reconstitution reveals the functional core of mammalian eIF3. The EMBO journal 26: 3373-83.

Maytal-kivity, V., Reis, N., Hofmann, K., and Glickman, M.H. 2002. proteins from eukaryotes and prokaryotes, is critical for Rpn11 function. Science and Technology 12: 1-12.

McCoy, A.J., Grosse-Kunstleve, R.W., Adams, P.D., Winn, M.D., Storoni, L.C., and Read, R.J. 2007. Phaser crystallographic software. Journal of applied crystallography 40: 658-674.

Methot, N., Pickett, G., Keene, J.D. and Sonenberg, N. 1996. In vitro RNA selection identifies RNA ligands that specifically bind to eukaryotic translation initiation factor 4B: the role of the RNA remotif. RNA 2(1):38-50.

Milon, P., Konevega, A.L., Peske, F., Fabbretti, A., Gualerzi, C.O., and Rodnina, M.V. 2007. Transient Kinetics, Fluorescence, and FRET in Studies of Initiation of Translation in Bacteria. Methods in Enzymology 430.

Minshall, N., Reiter, M.H., Weil, D., and Standart, N. 2007. CPEB interacts with an ovaryspecific eIF4E and 4E-T in early Xenopus oocytes. The Journal of biological chemistry 282: $37389-401$. 
Nielsen, K.H., Szamecz, B., Valásek, L., Jivotovskaya, A., Shin, B., and Hinnebusch, A.G. 2004. Functions of eIF3 downstream of 48S assembly impact AUG recognition and GCN4 translational control. The EMBO journal 23: 1166-77.

Nielsen, K.H., Valasek, L., Sykes, C., Jivotovskaya, A., and Hinnebusch, A.G. 2006. Interaction of the RNP1 Motif in PRT1 with HCR1 Promotes 40S Binding of Eukaryotic Initiation Factor 3 in Yeast. Molecular and cellular biology 26: 2984-2998.

Nika, J., Rippel, S., and Hannig, E.M. 2001. Biochemical analysis of the eIF2beta gamma complex reveals a structural function for eIF2alpha in catalyzed nucleotide exchange. The Journal of biological chemistry 276: 1051-6.

Nikonov, O., Stolboushkina, E., Nikulin, A., Hasenöhrl, D., Bläsi, U., Manstein, D.J., Fedorov, R., Garber, M., and Nikonov, S. 2007. New insights into the interactions of the translation initiation factor 2 from archaea with guanine nucleotides and initiator tRNA. Journal of molecular biology 373: 328-36.

Nissen, P., Kjeldgaard, M., Thirup, S., Palekhina, G., Reshetnikava, L., Clark, B.F., and Nyborg, J. 1993. Complexof Phe-tRNAPhe, EF-Tu, and a GTP Analog. Science 270: 1464-72.

Oberstrass, F.C. et al. 2005. Structure of PTB bound to RNA: specific binding and implications for splicing regulation. Science 309: 2054-7.

Passmore, L.A., Schmeing, T.M., Maag, D., Applefield, D.J., Acker, M.G., Algire, M.A., Lorsch, J.R., and Ramakrishnan, V. 2007. The eukaryotic translation initiation factors eIF1 and eIF1A induce an open conformation of the 40S ribosome. Molecular cell 26: 41-50.

Pedullà, N., Palermo, R., Hasenöhrl, D., Bläsi, U., Cammarano, P., and Londei, P. 2005. The archaeal eIF2 homologue: functional properties of an ancient translation initiation factor. Nucleic acids research 33: 1804-12.

Pestova, T.V., Lomakin, I.B., Lee, J.H., Choi, S.K., Dever, T.E., and Hellen, C.U. 2000. The joining of ribosomal subunits in eukaryotes requires eIF5B. Nature 403: 332-5.

Pestova, T.V., and Kolupaeva, V.G. 2002. The roles of individual eukaryotic translation initiation factors in ribosomal scanning and initiation codon selection. Genes \& development 16: 2906-22.

Phan, L., Schoenfeld, L.W., Valásek, L., Nielsen, K.H., and Hinnebusch, A.G. 2001. A subcomplex of three eIF3 subunits binds eIF1 and eIF5 and stimulates ribosome binding of mRNA and tRNA(i)Met. The EMBO journal 20: 2954-65.

Pick, E., Hofmann, K., and Glickman, M.H. 2009. PCI complexes: Beyond the proteasome, CSN, and eIF3 Troika. Molecular cell 35: 260-4.

Pisarev, A.V., Hellen, C.U., and Pestova, T.V. 2007. Recycling of eukaryotic posttermination ribosomal complexes. Cell 131: 286-99.

Pisarev, A.V., Kolupaeva, V.G., Pisareva, V.P., Merrick, W.C., Hellen, C.U., and Pestova, T.V. 2006. Specific functional interactions of nucleotides at key -3 and +4 positions flanking the initiation codon with components of the mammalian $48 \mathrm{~S}$ translation initiation complex. Genes \& development 20: 624-636. 
Pisarev, A.V., Kolupaeva, V.G., Yusupov, M.M., Hellen, C.U., and Pestova, T.V. 2008. Ribosomal position and contacts of mRNA in eukaryotic translation initiation complexes. The EMBO journal 27: 1609-21.

Pisarev, A.V., Skabkin, M.A., Pisareva, V.P., Skabkina, O.V., Rakotondrafara, A.M., Hentze, M.W., Hellen, C.U., and Pestova, T.V. 2010. The role of ABCE1 in eukaryotic posttermination ribosomal recycling. Molecular cell 37: 196-210.

Proweller, A., and Butler, J.S. 1997. Ribosome concentration contributes to discrimination against poly(A)- mRNA during translation initiation in Saccharomyces cerevisiae. The Journal of biological chemistry 272: 6004-10.

Pérard, J., Rasia, R., Medenbach, J., Ayala, I., Boisbouvier, J., Drouet, E., and Baudin, F. 2009. Human initiation factor eIF3 subunit $b$ interacts with HCV IRES RNA through its N-terminal RNA recognition motif. FEBS letters 583: 70-4.

Raught, B. and Gingras, A.C. 2007. Signaling to translation initiation. In: M. Mathews, N. Sonenberg and J.W.B. Hershey, Editors, Translational Control in Biology and Medicine, Cold Spring Harbor Laboratory Press, Cold Spring Harbor, NY, pp. 369-400.

Reibarkh, M. et al. 2008. Eukaryotic initiation factor (eIF) 1 carries two distinct eIF5-binding faces important for multifactor assembly and AUG selection. The Journal of biological chemistry 283: 1094-103.

Rodnina, M.V., and Wintermeyer, W. 2009. Recent mechanistic insights into eukaryotic ribosomes. Current opinion in cell biology 21: 435-43.

Saini, A.K., Nanda, J.S., Lorsch, J.R., and Hinnebusch, A.G. 2010. Regulatory elements in eIF1A control the fidelity of start codon selection by modulating tRNA(i)(Met) binding to the ribosome. Genes \& development 24: 97-110.

Sander, B., Golas, M.M. and Stark, H. 2003. Automatic CTF correction for single particles based upon multivariate statistical analysis of individual power spectra. Journal of Structural Biology 142(3):392-401.

Scheel, H., and Hofmann, K. 2005. Prediction of a common structural scaffold for proteasome lid, COP9-signalosome and eIF3 complexes. BMC bioinformatics 6: 71.

Schmitt, E., Blanquet, S., and Mechulam, Y. 2002. The large subunit of initiation factor aIF2 is a close structural homologue of elongation factors. The EMBO journal 21: 1821-32.

Schmitt, E., Naveau, M., and Mechulam, Y. 2010. Eukaryotic and archaeal translation initiation factor 2: a heterotrimeric tRNA carrier. FEBS letters 584: 405-12.

Schwechheimer, C., and Deng, X.W. 2001. COP9 signalosome revisited: a novel mediator of protein degradation. Trends in cell biology 11: 420-6.

Sha, Z., Brill, L.M., Cabrera, R., Kleifeld, O., Scheliga, J.S., Glickman, M.H., Chang, E.C., and Wolf, D.A. 2009. The eIF3 interactome reveals the translasome, a supercomplex linking protein synthesis and degradation machineries. Molecular cell 36: 141-52. 
Sharon, M., Taverner, T., Ambroggio, X.I., Deshaies, R.J., and Robinson, C.V. 2006. Structural Organization of the 19S Proteasome Lid: Insights from MS of Intact Complexes. PLoS Biology 4(8): e267.

Siridechadilok, B., Fraser, C.S., Hall, R.J., Doudna, J.a., and Nogales, E. 2005. Structural roles for human translation factor eIF3 in initiation of protein synthesis. Science 310: 1513-5.

Smolka, M.B., Albuquerque, C.P., Chen, S., and Zhou, H. 2007. Proteome-wide identification of in vivo targets of DNA damage checkpoint kinases. Proceedings of the National Academy of Sciences of the United States of America 104: 10364-9.

Sonenberg, N., and Hinnebusch, A.G. 2009. Regulation of translation initiation in eukaryotes: mechanisms and biological targets. Cell 136: 731-45.

Spahn, C.M., Gomez-Lorenzo, M.G., Grassucci, R.A., Jorgensen, R., Andersen, G.R., Beckmann, R., Penczek, P.A., Ballesta, J.P., and Frank, J. 2004. Domain movement of elongation factor eEF2 and the eukaryotic $80 \mathrm{~S}$ ribosome facilitate tRNA translocation. The EMBO journal 23: 1008-19.

Tedin, K., Moll, I., Grill, S., Resch, A., Graschopf, A., Gualerzi, C.O., and Bläsi, U. 1999. Translation initiation factor 3 antagonizes authentic start codon selection on leaderless mRNAs. Molecular microbiology 31: 67-77.

Triana-Alonso, F.J., Chakraburtty, K. and Nierhaus, K.H. 1995. The elongation factor 3 unique in higher fungi and essential for protein biosynthesis is an E site factor. Journal of Biological Chemistry 270: 20473-8.

Tsuda, K., Kuwasako, K., Takahashi, M., Someya, T., Inone, M., Terada, T., Kobayashi, N., Shiruzu, M., Kigawa, T., Tanaka, A., Sugano, S., Guenter, P., Muto, Y., and Yokoyama, S. 2009. Structural basis for the sequence-specific RNA-recognition mechanism of human CUGBP1 RRM3. Nucleic acids research 37: 5151-66.

Unbehaun, A., Borukhov, S.I., Hellen, C.U., and Pestova, T.V. 2004. Release of initiation factors from $48 \mathrm{~S}$ complexes during ribosomal subunit joining and the link between establishment of codon-anticodon base-pairing and hydrolysis of eIF2-bound GTP. Genes \& development 18: 3078-93.

Unbehaun, A., Marintchev, A., Lomakin, I.B., Didenko, T., Wagner, G., Hellen, C.U., and Pestova, T.V. 2007. Position of eukaryotic initiation factor eIF5B on the 80S ribosome mapped by directed hydroxyl radical probing. The EMBO journal 26: 3109-23.

Valasek, L., Nielsen, K.H., Zhang, F., Fekete, C.A., and Hinnebusch, A.G. 2004. Interactions of Eukaryotic Translation Initiation Factor 3 ( eIF3 ) Subunit NIP1/3c with eIF1 and eIF5 Promote Preinitiation Complex Assembly and Regulate Start Codon Selection. Molecular and cellular biology 24: 9437-9455.

Valásek, L., Hasek, J., Nielsen, K.H., and Hinnebusch, A.G. 2001. Dual function of eIF3j/Hcr1p in processing $20 \mathrm{~S}$ pre-rRNA and translation initiation. The Journal of biological chemistry 276: 43351-60. 
Valásek, L., Mathew, A.A., Shin, B., Nielsen, K.H., Szamecz, B., and Hinnebusch, A.G. 2003. The yeast eIF3 subunits TIF32/a, NIP1/c, and eIF5 make critical connections with the $40 \mathrm{~S}$ ribosome in vivo. Genes \& development 17: 786-99.

Valásek, L., Nielsen, K.H., and Hinnebusch, A.G. 2002. Direct eIF2-eIF3 contact in the multifactor complex is important for translation initiation in vivo. The EMBO journal 21: 588698.

Valásek, L., Phan, L., Schoenfeld, L.W., Valásková, V., and Hinnebusch, A.G. 2001. Related eIF3 subunits TIF32 and HCR1 interact with an RNA recognition motif in PRT1 required for eIF3 integrity and ribosome binding. The EMBO journal 20: 891-904.

van Heel, M., Harauz, G., Orlova, E.V., Schmidt, R., and Schatz, M. 1996. A new generation of the IMAGIC image processing system. Journal of structural biology 116: 17-24.

Vornlocher, H.P., Hanachi, P., Ribeiro, S., and Hershey, J.W. 1999. A 110-kilodalton subunit of translation initiation factor eIF3 and an associated 135-kilodalton protein are encoded by the Saccharomyces cerevisiae TIF32 and TIF31 genes. The Journal of biological chemistry 274: 16802-12.

Wei, N., Serino, G., and Deng, X. 2008. The COP9 signalosome: more than a protease. Trends in biochemical sciences 33: 592-600.

Wei, Z., Xue, Y., Xu, H., and Gong, W. 2006. Crystal structure of the C-terminal domain of S.cerevisiae eIF5. Journal of molecular biology 359: 1-9.

Woese, C.R. 1998. Archaeal translation initiation revisited: The initiation factor 2 and eukaryotic initiation factor 2B $\epsilon$ subunit families. Evolution 95: 3726-3730.

Yamamoto, Y., Singh, C.R., Marintchev, A., Hall, N.S., Hannig, E.M., Wagner, G., and Asano, K. 2005. The eukaryotic initiation factor (eIF) 5 HEAT domain mediates multifactor assembly and scanning with distinct interfaces to eIF1, eIF2, eIF3, and eIF4G. Proceedings of the National Academy of Sciences of the United States of America 102: 16164-9.

Yarunin, A., Panse, V.G., Petfalski, E., Dez, C., Tollervey, D., and Hurt, E.C. 2005. Functional link between ribosome formation and biogenesis of iron-sulfur proteins. The EMBO journal 24: 580-8.

Yatime, L., Mechulam, Y., Blanquet, S., and Schmitt, E. 2006. Structural switch of the gamma subunit in an archaeal aIF2 alpha gamma heterodimer. Structure 14: 119-28.

Yatime, L., Schmitt, E., Blanquet, S., and Mechulam, Y. 2004. Functional molecular mapping of archaeal translation initiation factor 2. The Journal of biological chemistry 279: 15984-93.

Yatime, L., Schmitt, E., Blanquet, S., and Mechulam, Y. 2005. Structure-function relationships of the intact aIF2alpha subunit from the archaeon Pyrococcus abyssi. Biochemistry 44: 8749-56.

Yu, Y., Marintchev, A., Kolupaeva, V.G., Unbehaun, A., Veryasova, T., Lai, S.C., Hong, P., Wagner, G., Hellen, C.U., and Pestova, T.V. 2009. Position of eukaryotic translation initiation factor eIF1A on the $40 \mathrm{~S}$ ribosomal subunit mapped by directed hydroxyl radical probing. Nucleic acids research 37: 5167-82. 
Zekri, L., Huntzinger, E., Heimstädt, S., and Izaurralde, E. 2009. The silencing domain of GW182 interacts with PABPC1 to promote translational repression and degradation of microRNA targets and is required for target release. Molecular and cellular biology 29: 622031.

Zhou, M., Sandercock, A.M., Fraser, C.S., Ridlova, G., Stephens, E., Schenauer, M.R., YokoiFong, T., Barsky, D., Leary, J.A., Hershey, J.W., Doudna, J.A., and Robinson, C.V. 2008. Mass spectrometry reveals modularity and a complete subunit interaction map of the eukaryotic translation factor eIF3. Proceedings of the National Academy of Sciences of the United States of America 105: 18139-44. 


\section{Acknowledgements}

First and foremost, I am very grateful and deeply indebted to Prof. Ralf Ficner. I express my sincere thanks for his guidance and support and for all the fruitful discussions and encouragement through the past four years.

I would like to thank:

All the former and present members of my thesis committee: Prof. Markus Wahl and Prof. George Sheldrick for great advices and helpful discussions during the first two years and Prof. Holger Stark and Prof. Marina Rodnina for sharing their expertise and brilliant advices in the last two years.

All the former and present members of the molecular structural biology department for their friendship and great working atmosphere. I am especially thankful to Dr. Carmen Rotte for helping me to start this journey and Bernhard Kuhle for great company. Many thanks to Dr. Achim Dickmanns, Dr. Piotr Neumann, Dr. Thomas Monecke, Dr. Eike Schulz and Dr. Bryan Wilkins for critical reading of parts of this thesis.

Dr. Pohl Milon and Dr. Florian Hauer for their expert advices and fruitful collaborations.

Dr. Bernhard Schmidt for mass spectrometric analyses.

Dr. Alan G. Hinnebusch, Prof. Jon R. Lorsch and Prof. Roland Lill for providing the yeast strains.

Dr. Steffen Burkhardt, Ivana Bacakova and Kerstin Grüniger for the outstanding organization of the International Molecular biology program and for all the daily helps during my stay in Germany.

Last, but not least I would like to express my deepest gratitude and sincerest thanks to my parents and my beloved wife, Homa. 


\section{Curriculum Vitae}

Sohail Khoshnevis

Born February $7^{\text {th }} 1983$ in Hamedan, Iran

AnnaStr. 5b, App. 113, 37075, Göttingen, Germany

Phone: +49-551-3914190

E-Mail: skhoshn@gwdg.de

Education

2006-present PhD Student, Department of Molecular Structural Biology, Georg-AugustUniversity, Göttingen, Germany

2005-2006 Georg-August-University, Göttingen, Germany International Max Planck Research School MSc/PhD Program Molecular Biology

2001-2005 Undergraduate Studies in Cellular and Molecular Biology Tehran University, Tehran, Iran

1997-2001 High School Diploma in Experimental Sciences Allameh Helli High School (NODET), Hamedan, Iran

Scholarships

2006-2009 Georg-Christoph Lichtenberg Stipend

2005-2006 International Max-Planck Research School Scholarship

2001-2005 University of Tehran Stipend for Exceptional Talents 


\section{Publications}

Khoshnevis, S., Gross, T., Rotte, C., Baierlein, C., Ficner, R., and Krebber, H. (2010). The ironsulphur protein RNase L inhibitor functions in translation termination. EMBO reports 11: 214-9.

Khoshnevis, S., Neumann, P., and Ficner, R. (2010). Crystal Structure of the RNA Recognition Motif of Yeast Translation Initiation Factor elF3b Reveals Differences to Human elF3b. PLoS ONE 5(9): e1278 